\title{
Relatively Open Gromov-Witten Invariants for Symplectic Manifolds of Lower Dimensions
}

\author{
Hai-Long Her
}

\begin{abstract}
Let $(X, \omega)$ be a compact symplectic manifold, $L$ be a Lagrangian submanifold and $V$ be a codimension 2 symplectic submanifold of $X$, we consider pseudoholomorphic maps from a Riemann surface with a fixed conformal structure and with boundary $(\Sigma, \partial \Sigma)$ to the pair $(X, L)$ satisfying Lagrangian boundary conditions and intersecting $V$. In some special cases, for instance, under the semi-positivity condition, we study the stable moduli space of such open pseudoholomorphic maps involving the intersection data. If $L \cap V=\emptyset$, we study the problem of orientability of the moduli space. Moreover, assume that there exists an anti-symplectic involution $\phi$ on $X$ such that $L$ is the fixed point set of $\phi$ and $V$ is $\phi$-anti-invariant, then we define the so-called "relatively open" invariants for the tuple $(X, \omega, V, \phi)$ if $L$ is orientable and $\operatorname{dim} X \leq 6$. If $L$ is nonorientable, we define such invariants under the condition that $\operatorname{dim} X \leq 4$ and some additional restrictions on the number of marked points on each boundary component of the domain.
\end{abstract}

\section{Contents}

1 Introduction

2 Stable open $(J, \nu)$-holomorphic maps 10

$3 \quad V$-compatible pair $(J, \nu)$

4 Moduli space of $V$-regular and $V$-stable maps 19

$4.1 \quad V$-regular open stable maps . . . . . . . . . . . . . . 20

4.2 Limits of $V$-regular open maps . . . . . . . . . . . . . . 22

4.3 The space of $V$-stable open maps . . . . . . . . . . . . . . 24

4.4 Gluing construction . . . . . . . . . . . . . . . . 27

5 Orietation $\quad 30$

5.1 Orienting the determinant line bundle over moduli space . . . . . . 30

5.2 Compatiblility with gluing . . . . . . . . . . . . . . 38

6 Involution and sign 41 
8 Proof of invariance 47

Appendix 53

\section{Introduction}

Gromov-Witten invariants $G W_{g, n}(X, A)$ for a symplectic manifold $(X, \omega)$, roughly speaking, count with sign the $(J, \nu)$-stable maps $u$ from a compact Riemann surface $\Sigma_{g, n}$ without boundary of genus $g$ with $n$ marked points to $(X, \omega)$, representing the homology class $A \in H_{2}(X)$ and satisfying some constraints, where $J$ is a $\omega$-compatible or $\omega$-tamed almost complex structure on $X$ and $\nu$ is an inhomogeneous perturbation satisfying the $J$-holomorphic map equation $\bar{\partial}_{J} u=\nu$. Such invariants originate from the seminal works of Gromov and Witten as well as Ruan's introducing Donaldson type invariants in symplectic category (see $[\mathrm{G}] \mathrm{W}] \mathbb{R}]$ ). The rigorous mathematical foundation of GW invariants was first established by Ruan-Tian [RT1] for semi-positive symplectic manifolds in 1993. Then many mathematicians contribute to the development and completion of such theme (see [FO [KM] LiT] RT2 [Si]). In many cases these numbers coincide with the enumerative invariants in algebraic geometry. In order to find effective ways of computing these invariants, Tian [Ti] first showed a rough description of studying the degeneration of rational curves under a symplectic degeneration, an operation of 'splitting the target'. Then Li-Ruan [LR and Ionel-Parker IP1] IP2] independently defined the so-called relative Gromov-Witten invariants with respect to a codimension 2 symplectic divisor $V$ and also established the symplectic connect sum formula for GW invariants. In the current paper, we construct certain relative GW invariants of open version (see Theorem 1.1 and 1.2 below) which in some sense count pseudo-holomorphic maps from Riemann surfaces with boundary and relative to a divisor.

For recent years, theoretic physicists have predicted the existence of enumerative invariants about pseudoholomorphic maps with Lagrangian boundary conditions by studying dualities for open strings (see [AKV] [OV]). Roughly speaking, let $L \subset X$ be a Lagrangian submanifold, such invariants would count (perturbed) pseudoholomorphic maps $u$ (or say $(J, \nu)$-maps satisfying equation $\bar{\partial}_{J} u=\nu$ ) from a Riemann surface with boundary $(\Sigma, \partial \Sigma)$ to $(X, L)$, representing a relative class $d \in H_{2}(X, L)$, such that the boundary is mapped in the Lagrangian submanifold. We denote by $\mathcal{M}_{k, l}(X, L, d)$ the moduli space of such maps $u$ with $k$ distinct marked points in $\partial \Sigma$ and $l$ distinct marked points in $\Sigma$, and its compactfication by $\overline{\mathcal{M}}_{k, l}(X, L, d)$. If we consider the special case that the domain surface with fixed conformal structure, to prescribe the domain surface, we will denote by $\mathcal{M}_{k, l}(\Sigma, X, L, d)$ and $\overline{\mathcal{M}}_{k, l}(\Sigma, X, L, d)$ the corresponding moduli spaces, respectively. Note that we have canonical evaluation maps at marked points

$$
e v b_{i}: \overline{\mathcal{M}}_{k, l}(X, L, d) \rightarrow L, \quad i=1, \cdots, k,
$$




$$
e v i_{j}: \overline{\mathcal{M}}_{k, l}(X, L, d) \rightarrow X, \quad j=1, \cdots, l .
$$

There are two main difficulties in defining such invariants: orientability of moduli space and codimension 1 bubbling-off phenomenon. Note, by [FOOO] Sil], that $\mathcal{M}_{k, l}(X, L, d)$ might be non-orientable. If $L$ is relatively spin, then Fukaya et al. proved that the moduli space is orientable. Katz-Liu $\mathrm{KL}] \mathrm{L}$ defined an open invariant under the much restricted assumption that there exists an additional $S^{1}$-action on the pair $(X, L)$. To define an orientation on the moduli space, they also assumed the Lagrangian submanifold $L$ is orientable and (relatively) spin. The orientability of moduli space seems important for Katz-Liu's definition of enumerative invariants. However, Solomon [So] showed that even if $L$ is not orientable, under the weaker assumption that $L$ is "relatively $\mathrm{Pin}^{ \pm}$" and some constraints on the number of boundary marked points, we still can obtain a canonical isomorphism from the orientation bundle of $\mathcal{M}_{k, l}(\Sigma, X, L, d)$ to the pull-backed line bundle from the orientation bundle of $L^{k}$ by the evaluation map $\prod_{i} e v b_{i}$. Although the moduli space $\mathcal{M}_{k, l}(\Sigma, X, L, d)$ may be non-orientable, the integral of $\operatorname{det}(T L)$-valued forms over $\mathcal{M}_{k, l}(\Sigma, X, L, d)$ would make sense.

On the other hand, we at present have no effective method to completely deal with the codimension 1 boundary of moduli space coming from the bubbling off discs. According to the discussion in AKV], in order to define an invariant independent of other choices, it seems necessary to introduce some additional parameter on $(X, L)$. For instance, the assumption in $[\mathrm{L}$ that there exists an $S^{1}$-action is used to cancel the effect of codimension 1 boundary of moduli space. In the paper [So, under the assumption that there exists an anti-symplectic involution

$$
\phi: X \rightarrow X, \quad \phi^{*} \omega=-\omega,
$$

such that $L=\operatorname{Fix}(\phi)$, and among other technical conditions, Solomon constructed the open invariants for $\operatorname{dim} X \leq 6$ if $L$ is orientable and, for $\operatorname{dim} X \leq 4$ even if $L$ might be non-orientable. Note that, from the viewpoint of real algebraic geometry, the assumption that symplectic manifold admits an anti-symplectic involution is more natural. Actually, in that paper, Solomon also showed that, for the genus zero domain surface and strongly semi-positive real symplectic manifold of dimension no more than 6 , his open invariants exactly coincide with twice of the Welschinger's invariants which can be regarded as a lower bound of the number of real rational curves in a real symplectic manifold(see [W1] W2 W3]). Independently, Cho [C], with different way of counting, also defined similar enumerative invariants for strongly semi-positive symplectic manifolds with $\operatorname{dim} X \leq 6$, genus $g=0$, and $L$ being relatively spin.

The aim of this article is to show a definition of "relatively open GromovWitten invariants" which intuitively would count stable maps $u:(\Sigma, \partial \Sigma) \rightarrow$ $(X, L)$ from a compact Riemann surface with boundary and with fixed conformal structure whose images intersect with a codimension 2 symplectic submanifold $V \subset X$, satisfying Lagrangian boundary conditions. In the current paper, to avoid technical complexity of constructing virtual cycle or Kuranishi structure, we do not deal with the most general case. Instead, we only consider the special 
case that all moduli spaces are of expected dimensions. That is we need the semipositivity assumptions 11 on both $(X, L)$ and $V$ (when $L_{V}=L \cap V \neq \emptyset$, we have to further assume that the pair $\left(V, L_{V}\right)$ is also semi-positive), or the assumption that each considered moduli space generically contains no multiply covered maps. These invariants are designed for a preparation of establishing open symplectic sum formulas, which will be used to compute open Gromov-Witten invariants of a symplectic connect sum of two symplectic manifolds $\left(X, L_{X}, V\right)$ and $\left(Y, L_{Y}, \bar{V}\right)$. More concretely, the symplectic sum is an operation that first removes $V$ and $\bar{V}$ from $X$ and $Y$, respectively, and then combines them into a new symplectic manifold $X \# Y$ with symplectic structures matching on the overlap region. A stable map into the sum is expected to be a pair of stable maps into the two sides which match in the middle. So the first step is to count stable maps in one side $X$ which record the intersection points with $V$ and multiplicities. At present, we only construct such invariants under the additional assumptions that $L$ is the fixed point set of an anti-symplectic involution, $\operatorname{dim} X \leq 6$ and $L \cap V=\emptyset$. However, for possible applications in the future, we will define the related moduli space and study the problem of orientability for more general situation. An axiomized formulation of the symplectic sum of open GW invariants has appeared in [LY.

Similar to the absolute case in [IP1, before considering such stable maps we have to extend $J$ and $\nu$ to the connect sum. The $V$-compatibility conditions imposed on the pair $(J, \nu)$ defined in Section 3 ensure that such an extension exists. However, these conditions do not always hold for generic $(J, \nu)$. Our relatively open invariants count stable maps for these special $V$-compatible pairs, which are different from the way of counting of the absolutely open GW invariants. Given such a special $V$-compatible pair $(J, \nu), V$ is a $J$-holomorphic submanifold, and a $(J, \nu)$-holomorphic map $u:(\Sigma, \partial \Sigma) \rightarrow\left(V, L_{V}\right)$ (if $L_{V}=L \cap V \neq \emptyset$ ) into $\left(V, L_{V}\right)$ is automatically a $(J, \nu)$-holomorphic map into $(X, L)$. Therefore, some domain components (closed or open) of stable maps maybe mapped entirely into $V$. Such maps are not transverse to $V$ and the moduli space of such maps may be of dimension larger than the expected dimension of $\mathcal{M}_{k, l}(\Sigma, X, L, d)$.

To avoid such non-transversal intersections, we restrict consideration to the so-called $V$-regular open stable maps which have no components mapped entirely into $V$. In the current paper, we only study these maps for the special case that $L \cap V=\emptyset$ which avoids the appearance of boundary intersections. The author plans to study the more difficult case $L \cap V \neq \emptyset$ in a separate paper. We note that such maps may intersect $V$ at finite many interior points with multiplicity. According to the ordering of these intersection points, the space of $V$-regular stable maps separates into components labeled by vector $\mathbb{s}=\left(s_{1}, \cdots, s_{\mathbb{1}}\right)$. The subscript $\mathbb{I}$ denotes the number of interior intersection points and $s_{j}$ denotes the multiplicity of the $j^{\text {th }}$ interior intersection point. Then in Section 4, we will study the moduli space $\mathcal{M}_{k, l}^{V}(\Sigma, X, L, d)$ of such $V$-regular stable maps. We will prove that each irreducible (see Definition [2.8) component $\mathcal{M}_{k, l, \mathbb{1}}^{V, \mathbb{s}}(\Sigma, X, L, d)$ of

\footnotetext{
${ }^{1}$ See the Definition 1.1
} 
$V$-regular maps is an orbifold with boundary whose dimension is expressed in (4.7). This applies to the semi-positive case since then those moduli spaces are generically irreducible.

In Sections 4.2 and 4.3, we consider the compactification of the moduli space of $V$-regular open maps - the space of $V$-stable open maps, of which each component is a subset of the closure of the $V$-regular space $\mathcal{M}_{k, l, \mathbb{1}}^{V, \mathbb{s}}(\Sigma, X, L, d)$ in the stable moduli space $\overline{\mathcal{M}}_{k, l+\mathbb{1}}(\Sigma, X, L, d)$. We will show that the irreducible part of this compactification is also an orbifold with boundary (corners). The key observation is that each sequence $\left\{u_{n}\right\}$ of $V$-regular open maps limits to a stable map $u$ with additional restriction. For instance, if the image of a component of such a limit map lies entirely in $V$, then along this component, we can find a section $\xi$ of the normal bundle of $V$, such that the elliptic equation $D^{N} \xi=0$ holds, where $D^{N}$ is the restriction of the linearization operator of $\bar{\partial}_{J, \nu}$ to the normal bundle of $V$. Stable maps with this additional restriction are called $V$-stable maps. Each component of the space of $V$-stable maps is denoted by $\overline{\mathcal{M}}_{k, l, \mathbb{1}}^{V, \mathrm{~s}}(\Sigma, X, L, d)$, which is the compactification of the space of $V$-regular open maps with frontier strata of codimension at least one. To analyze the convergence of sequences of $V$-regular maps, we refer to the renormalization technique used in [P1] and [T] for closed curves. For our open case, the parallel arguments can go through and we will omit the detail. Since the $V$-stable moduli space contains boundary or corners, in section 4.4 we will describe a gluing construction which gives the moduli space a local chart.

Then we come to define relative invariants for the case $L \cap V=\emptyset$. Let us introduce some more delicate notations. Recall that the maps we consider are $(j, J, \nu)$-holomorphic maps $u$ from a bordered Riemann surface $(\Sigma, \partial \Sigma)$ with fixed conformal structure $j$ to a symplectic-Lagrangian pair $(X, L)$ satisfying Lagrangian boundary conditions and representing a fixed relative homology class $d \in H_{2}(X, L)$. We suppose the boundary of $\Sigma$ has $m$ components, i.e. $\partial \Sigma=$ $\bigcup_{a=1}^{m}(\partial \Sigma)_{a}$ with each $(\partial \Sigma)_{a} \simeq S^{1}$. Moreover, we also require that the image of each boundary component represents a fixed homology class, i.e. $\left.u\right|_{(\partial \Sigma)_{a} *}\left(\left[(\partial \Sigma)_{a}\right]\right)=$ $d_{a} \in H_{1}(L)$. Suppose that there are $k_{a}$ marked points $z_{a 1}, \cdots, z_{a k_{a}}$ on each boundary component $(\partial \Sigma)_{a}$ and $l$ marked points $w_{1}, \cdots, w_{l}$ and additional $\mathbb{1}$ intersecting marked points $q_{1}, \cdots, q_{\mathbb{1}}$ on $\Sigma 2$ We reset

$$
\begin{gathered}
\mathbf{d}=\left(d, d_{1}, \cdots, d_{m}\right) \in H_{2}(X, L) \oplus H_{1}(L)^{\oplus m}, \\
\mathbf{k}=\left(k_{1}, \cdots, k_{m}\right), \quad|\mathbf{k}|=\sum_{a=1}^{m} k_{a}, \\
\mathbf{u}=(u, \vec{z}, \vec{w}, \vec{q}), \quad \vec{z}=\left(z_{a i}\right), \quad \vec{w}=\left(w_{j}\right), \quad \vec{q}=\left(q_{\jmath}\right) .
\end{gathered}
$$

Then we rewrite the moduli space of $V$-regular maps $\mathbf{u}$ by $\mathcal{M}_{\mathbf{k}, l, \mathbb{1}}^{V, \mathbb{s}}(\Sigma, X, L, \mathbf{d})$. The compactification $\overline{\mathcal{M}}_{\mathbf{k}, l, \mathbb{1}}^{V, \mathbb{s}}(\Sigma, X, L, \mathbf{d})$ is the space of $V$-stable open maps.

\footnotetext{
${ }^{2}$ To avoid some kind of bubbling, in this article, we require that if $\Sigma \simeq D^{2}, k>0$.
} 
There are some natural evaluation maps at those marked and intersection points

$$
\begin{gathered}
e v b_{a i}: \overline{\mathcal{M}}_{\mathbf{k}, l, \mathbb{1}}^{V, \mathbb{s}}(\Sigma, X, L, \mathbf{d}) \rightarrow L, \quad i=1, \cdots, k_{a}, a=1, \cdots, m, \\
e v i_{j}: \overline{\mathcal{M}}_{\mathbf{k}, l, \mathbb{1}}^{V, \mathbb{s}}(\Sigma, X, L, \mathbf{d}) \rightarrow X, \quad j=1, \cdots, l \\
e v i_{\jmath}^{I}: \overline{\mathcal{M}}_{\mathbf{k}, l, \mathbb{1}}^{V, \mathbb{s}}(\Sigma, X, L, \mathbf{d}) \rightarrow V, \quad \jmath=1, \cdots, \mathbb{1} .
\end{gathered}
$$

The first problem is the orientability of the moduli space. To get a more general result, we do not expect $\overline{\mathcal{M}}_{\mathbf{k}, l, \mathbb{1}}^{V, \$}(\Sigma, X, L, \mathbf{d})$ to have a canonical orientation, which means that there is a canonical orientation on the orientation line bundle $\operatorname{det}\left(\overline{\mathcal{M}}_{\mathbf{k}, l, \mathbb{1}}^{V, \mathbb{1}}(\Sigma, X, L, \mathbf{d})\right)$. Instead, we only want to construct an orientation on a modified line bundle

$$
\operatorname{det}\left(T \overline{\mathcal{M}}_{\mathbf{k}, l, \mathbb{1}}^{V, \mathbf{s}}(\Sigma, X, L, \mathbf{d})\right) \otimes \bigotimes_{a, i} e v b_{a i}^{*} \operatorname{det}(T L) .
$$

The conditions that ensure there exists such an orientation are that the Lagrangian submanifold $L$ is "relatively $\operatorname{Pin}^{ \pm}$" (see Definition 5.1) and some restrictions imposed on the boundary marked points. To state the following theorems, we recall

Definition 1.1 (1) A 2n-dimensional symplectic manifold $(M, \omega)$ is called semipositive if $\omega(\beta) \leq 0$ for any $\beta \in \pi_{2}(M)$ with $3-n \leq c_{1}(M)[\beta]<0$, where $c_{1}(M)$ is the first Chern class of $M$. (2) Let $L$ be a n-dimesional Lagrangian submanifold of $(M, \omega)$. A symplectic-Lagrangian pair $(M, L)$ is called semi-positive if $\omega(\beta) \leq 0$ for any $\beta \in \pi_{2}(M, L)$ with $3-n \leq \mu_{L}(\beta)<0$, where $\mu_{L}$ is the Maslov index.

In Section 5 we obtain the following result.

Theorem 1.1 Assume that both $(X, L)$ and $V$ are semi-positive or the moduli space $\overline{\mathcal{M}}_{\mathbf{k}, l, \mathbb{1}}^{V, \mathbb{s}}(\Sigma, X, L, \mathbf{d})$ generically contains no multiply covered map $\rrbracket^{3}, L$ is relatively Pin ${ }^{ \pm}$and fix a relatively Pin ${ }^{ \pm}$structure on $(X, L)$. If $L$ is not orientable, we assume $k_{a} \cong w_{1}\left(d_{a}\right)+1 \bmod 2$. If $L$ is orientable, fix an orientation. Then the relatively $\operatorname{Pin}^{ \pm}$structure on $(X, L)$ and the orientations of $L$ if it is orientable determines a canonical isomorphism

$$
\operatorname{det}\left(T \overline{\mathcal{M}}_{\mathbf{k}, l, \mathbb{1}}^{V, \mathbf{s}}(\Sigma, X, L, \mathbf{d})\right) \underset{\longrightarrow}{\longrightarrow} \bigotimes_{a, i} e v b_{a i}^{*} \operatorname{det}(T L) .
$$

Remark. By the Wu relation [MS], if $n \leq 3$ then $L$ is always Pin ${ }^{-}$.

Given the isomorphism (1.1), we can define the relatively open invariants as follows. Denote the images of marked points under evaluation maps by

$$
x_{a i}=u\left(z_{a i}\right), y_{j}=u\left(w_{j}\right), \mathbb{q}_{\jmath}=u\left(q_{\jmath}\right) .
$$

\footnotetext{
${ }^{3}$ See Definition 2.8
} 
Let $\Omega^{*}(L, \operatorname{det}(T L))$ denote differential forms on $L$ with values in $\operatorname{det}(T L)$, and let $\Omega^{*}(X)$ (resp. $\Omega^{*}(V)$ ) denote ordinary differential forms on $X$ (resp. $V$ ). Let $\alpha_{a i} \in \Omega^{n}(L, \operatorname{det}(T L)), a=1, \cdots, m ; i=1, \cdots, k_{a}$, represent the Poincaré dual of the point $x_{a i}$ in $H^{n}(L, \operatorname{det}(T L))$, which is the cohomology of $L$ with coefficients in the flat line bundle $\operatorname{det}(T L)$. Let $\gamma_{j} \in \Omega^{2 n}(X)$ represent the Poincaré dual of $y_{j}$ for $j=1, \cdots, l$. And let $\eta_{j} \in \Omega^{2 n-2}(V)$ represent the Poincaré dual of $\mathbb{q}_{j}$ for $\jmath=1, \cdots, \mathbb{1}$. Then we define

$$
\begin{aligned}
\mathscr{R} \mathscr{N} & :=\mathscr{R} \mathscr{N}(V, \mathbf{d}, \mathbf{k}, l, \mathbb{I}) \\
& =\int_{\overline{\mathcal{M}}_{\mathbf{k}, l, \mathbb{1}}^{V, \mathbb{s}}(\Sigma, X, L, \mathbf{d})} \bigwedge_{a, i} e v b_{a i}^{*}\left(\alpha_{a i}\right) \bigwedge_{j} e v i_{j}^{*}\left(\gamma_{j}\right) \bigwedge_{\jmath} e v i_{\jmath}^{I *}\left(\eta_{\jmath}\right) .
\end{aligned}
$$

The integral (1.2) makes sense because by Theorem 1.1, the integrand is a differential form taking values in the orientation line bundle of the $V$-stable moduli space. Denote by $\mu: H_{2}(X, L) \rightarrow \mathbb{Z}$ the Maslov index, and by $g$ the genus of the closed Riemann surface $\Sigma \cup_{\partial \Sigma} \bar{\Sigma}$ which is the complex double of $\Sigma$. From the dimensional calculation (4.7) we know that the integral above vanishes unless

$$
\begin{aligned}
(|\mathbf{k}|+2 l) n+2 \mathbb{I}(n-1)= & \mu(d)+n(1-g)+k \\
& +2(l+\mathbb{1}-\operatorname{deg} \Phi)-\operatorname{dim} A u t(\Sigma) .
\end{aligned}
$$

where $\operatorname{deg} \Phi=\sum_{j=1}^{\mathbb{I}} s_{j}$.

In general, the integral (1.2) might depend on the choice of the forms $\alpha_{a i}$, etc., because the codimension 1 boundary strata would contribute to the integral. To prove the invariance of the integral (1.2), we suppose that there exists an anti-symplectic involution $\phi$ on $X$, i.e. $\phi^{*} \omega=-\omega$, such that $L=\operatorname{Fix}(\phi)$ and the restriction of $\phi$ to $V$ is also an involution on the submanifold. It is not difficult to find an example for our setting. For example, let $(X, L)=\left(\mathbb{C} P^{1}, \mathbb{R} P^{1}\right)$, the symplectic form is induced by the Fubini-Study metric, the anti-symplectic involution $\phi$ is the complex conjugation, and $V=\left\{x^{+}, x^{-}\right\}$such that $x^{-} \neq x^{+}$ and $x^{-}=\phi\left(x^{+}\right)$.

Furthermore, suppose $\Sigma$ is biholomorphic to its conjugation $\bar{\Sigma}$, i.e. there exists an anti-holomorphic involution $c: \Sigma \rightarrow \Sigma$. Denote by $\mathcal{J}_{\omega}$ the set of $\omega$-tamed almost complex structures on $X$. Let $\mathcal{P}$ denote the set of $J$-anti-linear inhomogeneous perturbation terms. Define

$$
\mathcal{J}_{\omega, \phi}:=\left\{J \in \mathcal{J}_{\omega} \mid \phi^{*} J=-J\right\} .
$$

Now for each $J \in \mathcal{J}_{\omega, \phi}$, we denote by $\mathcal{P}_{\phi, c}^{J}$ the set of $\nu \in \mathcal{P}$ satisfying $d \phi \circ \nu \circ d c=\nu$. Denote

$$
\begin{gathered}
\mathbb{J}:=\left\{(J, \nu) \mid J \in \mathcal{J}_{\omega}, \nu \in \mathcal{P}\right\}, \\
\mathbb{J}_{\phi}:=\left\{(J, \nu) \mid J \in \mathcal{J}_{\omega, \phi}, \nu \in \mathcal{P}_{\phi, c}^{J}\right\} \subset \mathbb{J} .
\end{gathered}
$$

In Section 3, we will impose the $V$-compatibility conditions on the pair $(J, \nu)$, and denote by $\mathbb{J}^{V}$ (resp. $\mathbb{J}_{\phi}^{V}$ ) the set of all $V$-compatible pairs $(J, \nu) \in \mathbb{J}$ (resp. $\left.(J, \nu) \in \mathbb{J}_{\phi}\right)$. Fix $(J, \nu) \in \mathbb{J}_{\phi}^{V}$. Thus, from the $V$-stable $(J, \nu)$-holomorphic map 
$u:(\Sigma, \partial \Sigma) \mapsto(X, L)$ we can define its conjugate $V$-stable $(J, \nu)$-holomorphic map $\tilde{u}=\phi \circ u \circ c$ representing the homology class $[\tilde{u}]=-\phi_{*} d$, simply denoted by $\tilde{d}$. Denote $\tilde{\mathbf{d}}=\left(\tilde{d}, d_{1}, \cdots, d_{m}\right)$. So we have an induced map

$$
\phi^{\prime}: \overline{\mathcal{M}}_{\mathbf{k}, l, \mathbb{1}}^{V, \mathbb{s}}(\Sigma, X, L, \mathbf{d}) \rightarrow \overline{\mathcal{M}}_{\mathbf{k}, l, \mathbb{1}}^{V, \mathbb{s}}(X, L, \tilde{\mathbf{d}})
$$

given by

$$
\mathbf{u}=(u, \vec{z}, \vec{w}, \vec{q}) \mapsto \tilde{\mathbf{u}}=\left(\tilde{u},\left(\left.c\right|_{\partial \Sigma}\right)^{|\mathbf{k}|}(\vec{z}), c^{l}(\vec{w}), c^{\mathbb{1}}(\vec{q})\right)
$$

Define

$$
\begin{gathered}
\Omega_{\phi}^{*}(X):=\left\{\gamma \in \Omega^{*}(X) \mid \phi^{*} \gamma=\gamma\right\}, \\
\Omega_{\phi}^{*}(V):=\left\{\eta \in \Omega^{*}(V) \mid \phi^{*} \eta=\eta\right\} .
\end{gathered}
$$

Now in the integral (1.2) we take the forms $\gamma_{j} \in \Omega_{\phi}^{2 n}(X)$ and $\eta_{j} \in \Omega_{\phi}^{2 n-2}(V)$.

Then we study the lower dimensional cases, i.e. $n \leq 3$. If $d=\tilde{d}$, then using the method in this paper (see Sections 508), we can show that the integrals (1.2) are invariants of the tuple $(X, \omega, V, \phi)$. Roughly speaking, we can show that the map $\phi^{\prime}$ is an induced involution on the $V$-stable moduli space $\overline{\mathcal{M}}_{\mathbf{k}, l, \mathbb{1}}^{V, \Phi}(\Sigma, X, L, \mathbf{d})$. And using the isomorphism (1.1), we can assign a sign to each $V$-stable map (see Section [6). Then we can prove that on each codimension 1 boundary stratum, the induced involution $\phi^{\prime}$ is fixed point free and orientation reversing, i.e. changing the sign of each disc-bubbling stable map. Therefore, the contributions from the codimension 1 boundary of $\overline{\mathcal{M}}_{\mathbf{k}, l, \mathbb{1}}^{V, \mathbb{1}}(\Sigma, X, L, \mathbf{d})$ to the integrals (1.2) are eliminated. That implies the invariance.

However, for general situation, the relative homology class $d$ might not be $\phi$-anti-invariant, then $\phi^{\prime}$ might not be a map from the space of $V$-stable maps to itself. Thus the numbers $\mathscr{R} \mathscr{N}$ are not always well-defined invariants. So we have to modify the definition above.

The idea is to take all related moduli space together to eliminate the contributions from the codimension 1 boundaries to the integral. In fact, such boundarycanceling method has been used in [C].

For a homology class $\alpha \in H_{2}(X)$ and a relative class $\beta \in H_{2}(X, L)$, we say $\beta_{\mathbb{C}}=\mathbb{d}$ if there is a holomorphic map $u$ of class $\beta$ such that its complex double $u_{\mathbb{C}}$ represents the homology class $\alpha$. In this sense, we may say $\alpha=\beta_{\mathbb{C}}$ is the doubling of $\beta$. Note that $\beta_{\mathbb{C}}=(\tilde{\beta})_{\mathbb{C}}$. Denote by $\mathbb{d}=d_{\mathbb{C}}$ the homology class in $H_{2}(X)$ which is the doubling of $d$, and by $\bar{\alpha}=\left(\alpha_{a i}\right), \bar{\gamma}=\left(\gamma_{j}\right), \bar{\eta}=\left(\eta_{j}\right)$. Then we define

$$
\begin{aligned}
& \mathcal{I}:= \mathcal{I}_{X, \phi, g, \mathbb{d}, \mathbf{k}, l}^{V, \mathbf{\alpha}}(\bar{\alpha}, \bar{\gamma}, \bar{\eta}) \\
&= \sum_{\forall \beta: \beta_{\mathbb{C}}=\mathbb{d}} \sum_{j}:\left[\gamma_{j}\right]=\operatorname{PD}\left(\xi_{j}\right), \eta_{\jmath}:\left[\eta_{\jmath}\right]=\operatorname{PD}\left(\lambda_{\jmath}\right), \\
& \forall(\vec{x}, \vec{\xi}, \vec{\lambda}) \in \mathcal{R}
\end{aligned}
$$


where $\mathcal{R}$ is the space of real configurations (see (7.4) for detailed definition),

$$
\bar{\beta}=\left(\beta, d_{1}, \cdots, d_{m}\right) \in H_{2}(X, L) \oplus H_{1}(L)^{\oplus m},
$$

$\mathrm{PD}(\cdot)$ is the Poincaré dual of the point class. Now, we state our main result.

Theorem 1.2 Fix a conformal structure $j$ on a bordered Riemann surface $\Sigma$. Assume that $L \cap V=\emptyset$, and that $\operatorname{dim} X \leq 6$, if $L$ is not orientable, we assume that $\operatorname{dim} X \leq 4$ and $k_{a} \cong w_{1}\left(d_{a}\right)+1 \bmod 2$. If $(X, L)$ is semi-positive or each moduli space $\overline{\mathcal{M}}_{\mathbf{k}, l, \mathbb{1}}^{V, \mathbb{s}}(\Sigma, X, L, \bar{\beta})$ generically contains no $\phi$-multiply covered pseudoholomorphic man 4 , then the integers $\mathcal{I}=\mathcal{I}_{X, \phi, g, \mathbb{\mathbb { d }}, \mathbf{k}, l}^{V \text { are independent of the }}$ generic choice of pair $(J, \nu) \in \mathbb{J}_{\phi}^{V}$, the choice of conformal structure $j$, or the choice of forms $\alpha_{a i} \in \Omega^{n}(L, \operatorname{det}(T L)), \gamma_{j} \in \Omega_{\phi}^{2 n}(X), \eta_{\jmath} \in \Omega_{\phi}^{2 n-2}(V)$. Therefore, $\mathcal{I}$ are invariants of the tuple $(X, \omega, V, \phi)$.

Remark. In particular, if $d=\tilde{d}, \mathscr{R} \mathscr{N}:=\mathscr{R} \mathscr{N}(V, \mathbf{d}, \mathbf{k}, l, \mathbb{1})$ are invariants of the tuple $(X, \omega, V, \phi) . \mathscr{R} \mathscr{N}$ can be regarded as an extension of Solomon's definition of open GW invariants $N_{\Sigma, \mathbf{d}, \mathbf{k}, l}$ to the relative case. After the article was posted on arxiv, the author was informed by Welschinger that a definition of relatively open invariants for some special cases, i.e. cotangent bundles of 2 -sphere and real projective plane, has appeared in [W4].

Remark. In the proof of Theorem 1.1 and 1.2, we use the assumption that (generically) there is no $(\phi$-)multiply covered map. Actually, we believe, by using the virtual cycle techniques to the case of open maps or the equivariant Kuranishi structure (see, for example, section 7 of [So]) or the expected polyfold techniques being developed by Hofer et al, that neither such assumption nor the semi-positivity assumption is necessary and the Theorem 1.1 and 1.2 holds for general case.

In Section 5, we deal with the problem of orientation and derive the conclusion of Theorem 1.1. Then in Section [6, we assign a sign to each $V$-stable map and study how the induced action by involution changes the sign. The last two sections devote to the proof of Theorem 1.2. The next Sections 2 is preparation for succeeding discussion, we show the definition of stable open $(J, \nu)$-holomorphic maps and describe the moduli space of such maps. For the convenience of the reader, in Appendix we review some definitions and important conclusions in $\mathrm{So}$ about the orientation of determinant of real linear Cauchy-Riemann operator.

Acknowledgments. The author thanks the Institute of Mathematical Science at Nanjing University, the Department of Mathematics at Princeton University, the IHESS and the BICMR for hospitality and support, part of this work was done when he was working at or visiting these institutions. He also thanks Gang Tian for inspiring conversations on relative invariants, An-Min Li, Thomas Parker,

\footnotetext{
${ }^{4} \mathrm{~A}$ nonconstant map $u:(\Sigma, \partial \Sigma) \rightarrow(X, L)$ is called $\phi$-multiply covered or not $\phi$-somewhere injective if there exists a regular point $z \in \Sigma$ such that either $u(z) \in u(\Sigma \backslash\{z\})$ or $u(z) \in \operatorname{Im}(\phi \circ u)$.
} 
Jack Solomon and Jean-Yves Welschinger for explaining to him some points in the definitions of relative GW invariants, open GW invariants and real enumerative invariants, respectively. Thanks also go to Bohui Chen, Xiaojun Chen, Huijun Fan, Jianxun Hu, Guangcun Lu and Shanzhong Sun for helpful conversations, and Scott Kenney for providing direct help for his working and living in Princeton. He is very grateful to Yiming Long, Jiangong You for their long-term encouragement, and Haihong Lin for special financial support in the spring of 2008 when he had no income. He wants to thank referees for their constructive comments and giving him many nice suggestions.

\section{Stable open $(J, \nu)$-holomorphic maps}

In this section, we recall the definition of stable open $(J, \nu)$-holomorphic maps, and describe the moduli space of such maps. The domains of such stable open maps are bordered Riemann surface i.e. compact Riemann surface with boundary, smooth or allowing nodal singularities. The boundary is mapped in a Lagrangian submanifold.

In the following, both marked points and double points (or singular points or nodes) are called special points. And we always fix a conformal structure on the open domain curve $\Sigma$, which is a bordered compact Riemann surface. We say such curve is of genus $g$ if the closed Riemann surface $\Sigma \cup_{\partial \Sigma} \bar{\Sigma}$, which is the complex double of $\Sigma$ and also denoted by $\Sigma_{\mathbb{C}}$ (see [AG]), is of genus $g$. Denote the genus of the closed surface $\Sigma / \partial \Sigma$ by $g_{0}$.

Assume that there are altogether $m$ boundary components, i.e. $\partial \Sigma=\bigcup_{a=1}^{m}(\partial \Sigma)_{a}$. We say $\Sigma$ is of topological type $\left(g_{0}, m\right)$.

Definition 2.1 An automorphism of a bordered Riemann surface $\Sigma$ is a diffeomorphism $\varphi: \Sigma \rightarrow \Sigma$ preserving the conformal structure and the ordering of the boundary components. The set of all automorphism of $\Sigma$ is denoted by $\operatorname{Aut}(\Sigma)$. We say $\Sigma$ is stable if $\operatorname{Aut}(\Sigma)$ is finite.

Denote by $\Sigma_{\mathbb{C}}$ the complex double of $\Sigma$, which is a closed Riemann surface with an antiholomorphic involution $\sigma$. If $\varphi: \Sigma \rightarrow \Sigma$ is an automorphisim, then its complex double $\varphi_{\mathbb{C}}: \Sigma_{\mathbb{C}} \rightarrow \Sigma_{\mathbb{C}}$ is an automorphism of $\left(\Sigma_{\mathbb{C}}, \sigma\right)$. This provides a natural inclusion $\operatorname{Aut}(\Sigma) \subset \operatorname{Aut}\left(\Sigma_{\mathbb{C}}, \sigma\right)$. The following statements are equivalent:

- $\Sigma$ is stable, i.e., $\operatorname{Aut}(\Sigma)$ is finite.

- $\Sigma_{\mathbb{C}}$ is stable.

- The genus $g=2 g_{0}+m-1$ of $\Sigma_{\mathbb{C}}$ is bigger than one.

- The Euler characteristic $\chi(\Sigma)=2-2 g_{0}-m$ of $\Sigma$ is negative.

Definition 2.2 A prestable bordered Riemann surface with fixed conformal structure is either a smooth bordered Riemann surface or the union of a smooth bordered Riemann surface with finite sphere and disc bubbles. 
In this paper, we only consider the prestable bordered Riemann surfaces with fixed conformal structure, and simply call them prestable bordered Riemann surfaces.

Definition 2.3 An automorphism of a prestable bordered Riemann surface $\Sigma$ of topological type $\left(g_{0}, m\right)$ with $(\mathbf{k}, l)$ marked points

$$
(\Sigma, \partial \Sigma, \vec{z}, \vec{w})=\left(\Sigma,\left\{(\partial \Sigma)_{a}\right\}_{a=1}^{m},\left\{z_{a 1}, \cdots, z_{a k_{a}}\right\}_{a=1}^{m}, w_{1}, \cdots, w_{l}\right)
$$

is an automorphism of $\Sigma$ preserving all marked points. The set of all automorphism is denoted by $\operatorname{Aut}\left(\Sigma_{\mathbf{k}, l}\right)$ (or simply by $\operatorname{Aut}(\Sigma)$ ). A prestable $(\mathbf{k}, l)$-marked bordered Riemann surface $\Sigma$ is stable if $\operatorname{Aut}\left(\Sigma_{\mathbf{k}, l}\right)$ is finite.

Definition 2.4 A $(\mathbf{k}, l)$-marked bordered bubble domain curve $\Sigma$ of type $\left(g_{0}, m\right)$, which is also called a prestable $(\mathbf{k}, l)$-marked bordered Riemann surface and where the vector $\mathbf{k}=\left(k_{1}, \cdots, k_{m}\right)$, is a finite connected union of smooth oriented compact surfaces $\Sigma_{i}$, at least one surface with boundary, joined at interior or boundary double points together with $k_{a}$ distinct marked points in the boundary $(\partial \Sigma)_{a}$, $a=1, \cdots, m$, and $l$ distinct interior marked points, none of which are double points. The $\Sigma_{i}$, with their special points, are of two types:

(1) stable components, and

(2) unstable components, which are unstable sphere bubbles or unstable disc bubbles.

And there must be at least one stable component.

There exists a natural stablization map

$$
\text { st }: \Sigma \rightarrow \widehat{\Sigma}
$$

that collapses the unstable components to points, thus we get a connected domain $\widehat{\Sigma}=\operatorname{st}(\Sigma)$ which is a stable genus $g$ open curve.

Bordered bubble domains can be constructed from a stable bordered Riemann surface $\Sigma_{0}$ by replacing points by finite chains of 2 -spheres or 2-discs or their combination. Alternatively, they can be obtained from a smooth Riemann surface $\Sigma_{0}$ by pinching a set of nonintersecting embedded circles in the interior of $\Sigma_{0}$ and (or) a set of half-circles in $\Sigma_{0}$ with centers in the boundary $\partial \Sigma_{0}$. The latter viewpoint can be formalized as follows. Assume that there are $b_{a}$ double points on each boundary component $(\partial \Sigma)_{a}, a=1, \cdots, m$, and there are $d$ interior double points. Denote by $\mathbf{b}=\left(b_{1}, \cdots, b_{m}\right)$.

Definition 2.5 A resolution of $\left(g_{0}, m\right)$-type $(\mathbf{k}, l)$-marked bordered bubble domain $\Sigma$ with $(\mathbf{b}, d)$-double points is a smooth bordered Riemann surface with genus $g, d$ embedded circles $\gamma_{c}$ in the interior part and $b_{a}$ embedded half-circles $\gamma_{a h}$ with centers in each $\left(\partial \Sigma_{0}\right)_{a}, a=1, \cdots, m$, (any two of distinct circles or half-circles are disjoint), and the $(\mathbf{k}, l)$-marked points are apart from $\gamma_{a h}$ and $\gamma_{c}$, together with a resolution map

$$
\mathscr{R}: \Sigma_{0} \rightarrow \Sigma
$$


which respects orientation and marked points, takes each $\gamma_{c}\left(\right.$ resp. $\left.\gamma_{a h}\right)$ to an interior (resp. boundary) double point of $\Sigma$, and restricts to a diffeomorphism from the complement of $\bigcup \bar{\gamma}_{a h} \cup \bar{\gamma}_{c}$ in $\Sigma$ to the complement of the double points, where $\bar{\gamma}_{a h}$ (resp. $\bar{\gamma}_{c}$ ) denotes the closure of half-disc (resp. disc) contained by $\gamma_{a h}$ $\left(\right.$ resp. $\left.\gamma_{c}\right)$.

We next define $(J, \nu)$-holomorphic maps from bubble domains. Such maps depend on the choice of an $\omega$-compatible (tamed) almost complex structure $J \in$ $\mathcal{J}_{\omega}$ and an inhomogeneous perturbation $\nu$ to the Cauchy-Riemann equation. Let $\mathcal{B}$ be a parameter space which will be specified later. Let $\pi_{i}, i=1,2$, denote the projection from $\Sigma \times X \times \mathcal{B}$ to the $i^{\text {th }}$ factor and let $\pi_{i}^{\prime}$ denote the restriction of $\pi_{i}$ to $\partial \Sigma \times L \times \mathcal{B}$. We define the inhomogeneous term to be the section

$$
\nu \in \Gamma\left(\Sigma \times X \times \mathcal{B}, \operatorname{Hom}\left(\pi_{1}^{*} T \Sigma, \pi_{2}^{*} T X\right)\right)
$$

such that

(1) $\nu$ is $\left(j_{\Sigma}, J\right)$-anti-linear: $\nu \circ j_{\Sigma}=-J \circ \nu$;

(2) $\left.\nu\right|_{\partial \Sigma \times L \times \mathcal{B}}$ carries a sub-bundle $\pi_{1}^{\prime *} T \partial \Sigma \subset \pi_{1}^{*} T \Sigma$ to the sub-bundle $\pi_{2}^{\prime *}(T L) \subset$ $\pi_{2}^{*} T X 5$

Denote by $\mathcal{P}$ the set of all such inhomogeneous terms. Let $\mathbb{J}$ denote the space of such pairs $(J, \nu)$. The parameter space $\mathcal{B}$ is often taken to be the ambient space of relevant pseudo-holomorphic maps. For instance, in this section, let $\mathcal{B}=B^{1, p}(\Sigma, L, \mathbf{d})$ be the Banach manifold of $W^{1, p}$ maps $u:(\Sigma, \partial \Sigma) \rightarrow(X, L)$ such that $u_{*}([\Sigma, \partial \Sigma])=d$ and $\left.u\right|_{(\partial \Sigma)_{a} *}\left(\left[(\partial \Sigma)_{a}\right]\right)=d_{a}$. In section 3 , we will take $\mathcal{B}$ as the space (3.2).

Definition 2.6 $A(J, \nu)$-holomorphic open map from a bordered bubble domain curve $(\Sigma, \partial \Sigma)$ with complex structure $j_{\Sigma}$ is a map

$$
u:(\Sigma, \partial \Sigma) \rightarrow(X, L)
$$

such that, on each component $\Sigma_{i}$ of $\Sigma, u$ is a solution of the inhomogeneous Cauchy-Riemann equation

$$
\bar{\partial}_{J} u=\frac{1}{2}\left(d u+J \circ d u \circ j_{\Sigma}\right)=\nu(\cdot, u(\cdot), \mathbf{u}),
$$

or equivalently,

$$
\bar{\partial}_{(J, \nu)} u=0
$$

where $\bar{\partial}_{(J, \nu)}$ denotes the perturbed nonlinear elliptic operator $\frac{1}{2}\left(d+J \circ d \circ j_{\Sigma}\right)-\nu$. In particular, $\bar{\partial}_{J} u=0$ on each unstable bubble.

Remark. It is not difficult to show that the operator $\bar{\partial}_{(J, \nu)}$ gives rise to an elliptic boundary value problem. We refer to the Lemma 4.1 in [So].

\footnotetext{
So.

${ }^{5}$ Note here our setting, i.e. the following equation (2.3), is a little different from the one in
} 
The symplectic area of the image is the number

$$
A(u)=\int_{u(\Sigma)} \omega=\int_{\Sigma} u^{*} \omega
$$

which depends only on the homology class of the curve modulo its boundary. And the energy of $u$ is

$$
E(u)=\frac{1}{2} \int_{\Sigma}|d u|_{J, \mu}^{2} d \mu
$$

where $|\cdot|_{J, \mu}$ is the norm defined by the metric on $X$ determined by $J$ and the metric $\mu$ on the domain. For $(J, 0)$-holomorphic maps, $E(u)=A(u)$.

We also define a modified energy $\mathbb{E}$ componentwise

$$
\mathbb{E}\left(u_{i}\right)= \begin{cases}1+\frac{1}{2} \int_{\Sigma_{i}}\left|d u_{i}\right|_{J, \mu}^{2} d \mu, & \Sigma_{i} \text { is stable and } u_{i} \text { is not a ghost; } \\ \frac{1}{2} \int_{\Sigma_{i}}\left|d u_{i}\right|_{J, \mu}^{2} d \mu, & \Sigma_{i} \text { is unstable. }\end{cases}
$$

And

$$
\mathbb{E}(u)=\sum_{i} \mathbb{E}\left(u_{i}\right)
$$

Now we come to the definition of stable map

Definition 2.7 $A(J, \nu)$-holomorphic map $u$ is stable if each of its component maps $u_{i}=\left.u\right|_{\Sigma_{i}}$ has positive modified energy i.e. $\mathbb{E}\left(u_{i}\right)>0$ for each $i$.

That is to say, either each component $\Sigma_{i}$ of the domain is stable, or else the image of $\left(\Sigma_{i}, \partial \Sigma_{i}\right)$ carries a nontrivial homology class. We have

Lemma 2.1 Let $(X, \omega)$ be a compact symplectic manifold and $L$ be a compact Lagrangian submanifold. Then

(1) every $(J, \nu)$-holomorphic map has $\mathbb{E}(u) \geq 1$.

(2) There exists a constant $\hbar>0$ such that for every component $u_{i}$ of every stable $(J, \nu)$-holomorphic map $u:(\Sigma, \partial \Sigma) \rightarrow(X, L)$ with Lagrangian boundary condition, we have

$$
\mathbb{E}\left(u_{i}\right)>\hbar
$$

Proof. (1) The conclusion is direct since at least one component is stable.

(2) On the stable components, we have $\mathbb{E}\left(u_{i}\right) \geq 1$. On the unstable components, since $u_{i}$ is a $J$-holomorphic map, the Proposition 4.1.4 of [McS] implies there exists $\hbar>0$ depending only on $(X, J)$ such that

$$
\mathbb{E}\left(u_{i}\right)=E\left(u_{i}\right)=\frac{1}{2} \int_{\Sigma_{i}}\left|d u_{i}\right|_{J, \mu}^{2} d \mu>\hbar,
$$

provided the images of $\left(\Sigma_{i}, \partial \Sigma_{i}\right)$ carries a nontrivial homology class. Otherwise, if $u_{i}$ represents a trivial homology class, then $\mathbb{E}\left(u_{i}\right)=E\left(u_{i}\right)=A\left(u_{i}\right)=\omega \cap$ $\left[u_{i}\left(\Sigma_{i}\right)\right]=0$, contrary to the definition of stable map.

The following Gromov Convergence Theorem, which is also called Compactness Theorem, is the important fact about $(J, \nu)$-holomorphic maps. It means 
that every sequence of $(J, \nu)$-holomorphic maps from a smooth (bordered) domain has a subsequence which converges modulo automorphism to a stable map. Various forms of such convergence theorem have been proved for closed curves (c.f. G], PW], RT1, [Y], etc.). For open curves, we refer to [FOOO, [L, McS]. Moreover, we assume $|\mathbf{k}|>\mathbf{0}$ to exclude some exceptional case 6 .

Theorem 2.1 (Bubble Convergence). Let $(X, \omega)$ be a compact symplectic manifold, $L \subset X$ be a Lagrangian submanifold. Given any sequence $\left\{u_{j}\right\}$ of $(\mathbf{k}, l)$ marked $\left(J_{j}, \nu_{j}\right)$-holomorphic maps from a bordered Riemann surface $\Sigma_{0}$ satisfying Lagrangian boundary conditions, with $\mathbb{E}\left(u_{j}\right)<E_{0}$ and $\left(J_{j}, \nu_{j}\right) \rightarrow(J, \nu)$ in $C^{k}$, $k \geq 0$, then we can obtain a subsequence and

(1) a $(\mathbf{k}, l)$-marked bordered bubble domain $\Sigma$ with resolution $\mathscr{R}: \Sigma_{0} \rightarrow \Sigma$, and

(2) automorphisms $\varphi_{j}$ of $\Sigma_{0}$ preserving the orientation and the marked points, such that the modified subsequence $\left\{u_{j} \circ \varphi_{j}\right\}$ converges to a limit

$$
\Sigma_{0} \stackrel{\mathscr{R}}{\longrightarrow} \Sigma \stackrel{u}{\longrightarrow} X
$$

where $u$ is a stable $(J, \nu)$-holomorphic map. This convergence is in $C^{0}$, and in $C^{k}$ on compact sets not intersecting the collapsing curves $\gamma_{a h}$ and $\gamma_{c}$ of the resolution $\mathscr{R}$, and the area (2.4) and energy (2.7) are preserved in the limit.

Given a bordered bubble domain curve with fixed complex structure $(\Sigma, \partial \Sigma)$. Denote the space of equivalence classes of stable $(\mathbf{k}, l)$-marked open pseudoholomorphic maps with Lagrangian boundary conditions representing the homology class $\mathbf{d}$ by $\overline{\mathcal{M}}_{\mathbf{k}, l}(\Sigma, X, L, \mathbf{d})$ which is just the Gromov compactification of $\mathcal{M}_{\mathbf{k}, l}(\Sigma, X, L, \mathbf{d})$.

Definition 2.8 We say a map $u:(\Sigma, \partial \Sigma) \rightarrow(X, L)$ is multiply covered if there does not exist a point $z \in \Sigma$ such that

$$
d u(z) \neq 0, \quad \text { and } \quad u(z) \notin u(\Sigma \backslash\{z\}) .
$$

A map is called irreducible or somewhere injective if it is not multiply covered. The moduli space $\mathcal{M}_{\mathbf{k}, l}(\Sigma, X, L, \mathbf{d})$ or $\overline{\mathcal{M}}_{\mathbf{k}, l}(\Sigma, X, L, \mathbf{d})$ is called irreducible if it contains no multiply covered pseudoholomorphic map.

Remark. As mentioned in the introduction, if there exists an anti-symplectic involution $\phi$ on the symplectic manifold $X$ such that $L=\operatorname{Fix}(\phi)$, there is a modified definition of the so-called $\phi$-multiply covered map, we refer to the footnote of the Theorem 1.2. The multiply covered maps are often singular points in the moduli space $\mathcal{M}_{\mathbf{k}, l}(\Sigma, X, L, \mathbf{d})$. For keeping the paper in suitable length, we will avoid dealing with these maps in the present paper.

Let $\overline{\mathcal{M}}_{\mathbf{k}, l}(\Sigma, X, L, \mathbf{d})^{*}$ be the space of irreducible stable open $(J, \nu)$-maps. The following theorem is direct from Theorem 2.1, the index theorem and the standard arguments of transversality theorem

\footnotetext{
${ }^{6}$ When $|\mathbf{k}|=\mathbf{0}$, it turns out that the standard stable map moduli space might be not compact. See sections 3.8 and 7.4 of [FOOO] for discussion of such case.
} 
Theorem 2.2 Fix a conformal structure $j$ on $\Sigma$ (forgetting $(\mathbf{k}, l)$ marked points), denote the group of automorphisms of $\Sigma$ by Aut $(\Sigma)$. We assume $|\mathbf{k}|>\mathbf{0}$, then

(1) $\overline{\mathcal{M}}_{\mathbf{k}, l}(\Sigma, X, L, \mathbf{d})$ is compact and Hausdorff, and there exists a continuous evaluation map

$$
\mathrm{ev}: \overline{\mathcal{M}}_{\mathbf{k}, l}(\Sigma, X, L, \mathbf{d}) \longrightarrow X^{l} \times L^{|\mathbf{k}|}
$$

(2) For generic $(J, \nu) \in \mathbb{J}, \overline{\mathcal{M}}_{\mathbf{k}, l}(\Sigma, X, L, \mathbf{d})^{*}$ is a manifold with boundary (corners) of dimension

$$
\operatorname{dim} \overline{\mathcal{M}}_{\mathbf{k}, l}(\Sigma, X, L, \mathbf{d})^{*}=\mu(d)+n(1-g)+k+2 l-\operatorname{dim} \operatorname{Aut}(\Sigma) .
$$

Remark. The conclusions of the theorem above did not appear exactly in the literature, while there exist related results in some different settings. In [FOOO] and $[\mathrm{L}$, they show that, for general symplectic manifold, the stable moduli space of open unperturbed $J$-maps is compact and Hausdorff and carries the smooth Kuranishi structure, from which they respectively constructed the virtual fundamental chain (see Theorem 2.1.29.-32. of [FOOO], or sections 5.3 and 7.1 of [L]). In [McS, they only deal with the genus 0 unperturbed $J$-maps (in fact, they claim that their arguments go through to higher genus case with fixed complex structure), and conclude that the irreducible part of the moduli space is generically a manifold. In [RT1] [RT2] and [LiT], they study the closed $(J, \nu)$-maps in semi-positive and general symplectic manifolds, respectively. Their arguments may apply to our open case with minor modifications, since here we just describe the stable moduli space instead of defining invariants. We will not give a proof of the theorem above here, and refer to literature mentioned above and a similar arguments, corresponding to Theorem 2.2 (2), in the proof of Lemma 4.1 .

\section{$3 \quad V$-compatible pair $(J, \nu)$}

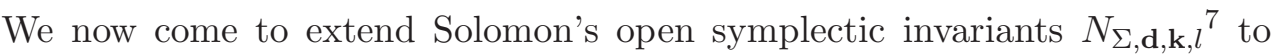
open invariants of $(X, \omega, \phi)$ relative to a codimension 2 symplectic submanifold $V$ such that $\left.\phi\right|_{V}$ is also an anti-symplectic involution on $\left(V,\left.\omega\right|_{V}\right)$. Recall the assumption that $L=\operatorname{Fix}(\phi) \neq \emptyset$ is a Lagrangian submanifold. Note that $V \cap L$ might be empty set. If $V \cap L=\operatorname{Fix}\left(\left.\phi\right|_{V}\right) \neq \emptyset$, then we denote by $L_{V}=V \cap L$ which is a Lagrangian submanifold of $\left(V,\left.\omega\right|_{V}\right)$. We consider open curves generically intersect $V$ in a finite collection of points. Such relative open invariants will count these open curves satisfying some constraints. In particular, if $L \cap V=\emptyset$, we will not encounter extra codimension 1 boundary of moduli space except the moduli space of pseudoholomorphic maps with a bubble disc. If $\operatorname{dim} L \leq 3$, under some assumptions, we will define relatively open invariants for domain curves of any genus with fixed conformal structures.

Let us reset our notations for new discussion. Suppose $\operatorname{dim} X=2 n$. We denote still by $(\Sigma, \partial \Sigma)$ the domain Riemann surface with $m$ boundary components and with fixed conformal structure $j_{\Sigma}$. That means we also don't deal with the

\footnotetext{
${ }^{7}$ Also one can define the Solomon-type open invariant for more general case, for one such generalization see $[\underline{\mathrm{H}}$.
} 
case that the degenerations of $\Sigma$ may occur. Denote by $g$ the genus of the closed Riemann surface $\Sigma \cup_{\partial \Sigma} \bar{\Sigma}$ obtained by doubling $\Sigma$. If $\Sigma$ is a closed Riemann surface then $g$ is just the genus of itself. Assume that there are $k_{a}$ distinct marked points $\left\{z_{a 1}, \cdots, z_{a k_{a}}\right\}$ on the boundary component $(\partial \Sigma)_{a}, a=1, \cdots, m$, and $l$ distinct interior marked points $\left\{w_{1}, \cdots, w_{l}\right\}$. Denote by $\mathbf{k}=\left(k_{1}, \cdots, k_{m}\right)$. Additionally, we assume that there are $\mathbb{k}_{a}$ marked points $\left\{p_{a 1}, \cdots, p_{a \mathbb{k}_{a}}\right\}$ on each boundary component $(\partial \Sigma)_{a}, a=1, \cdots, m$, which are different from $\left\{z_{a 1}, \cdots, z_{a k_{a}}\right\}$ and are mapped to the intersection points of $V$ and the image of our open curve $u:(\Sigma, \partial \Sigma) \rightarrow(X, L)$ if $V \cap L \neq \emptyset$. Denote by $\mathbb{k}_{\mathbf{k}}=\left(\mathbb{k}_{1}, \cdots, \mathbb{k}_{m}\right)$. Also, we assume there are $\mathbb{1}$ interior marked points $\left\{q_{1}, \cdots, q_{\mathbb{1}}\right\}$ which are different from $\left\{w_{1}, \cdots, w_{l}\right\}$ and are mapped to the intersection points of $V$ and the image of $u$. For given homology class $\mathbf{d}=\left(d, d_{1}, \cdots, d_{m}\right) \in H_{2}(X, L) \oplus H_{1}(L)^{\oplus m}$, and given a pair $(J, \nu)$, denoted by $\mathcal{M}_{\mathbf{k}, l, \mathbb{1}}(\Sigma, X, L, \mathbf{d})$ the moduli space of $(\mathbf{k}, l, \mathbb{1})$-marked $(J, \nu)$-pseudoholomorphic maps such that $u_{*}([\Sigma, \partial \Sigma])=d$ and $\left.u\right|_{(\partial \Sigma)_{a} *}\left(\left[(\partial \Sigma)_{a}\right]\right)=$ $d_{a}$, i.e. representing $\mathbf{d}$, which is the zero set of

$$
\Phi(u, J, \nu)=\bar{\partial}_{(J, \nu)} u=\bar{\partial}_{J} u-\nu=\frac{1}{2}\left(d u+J \circ d u \circ j_{\Sigma}\right)-\nu .
$$

We denote the ambient space, which is the Sobolev completion of $\mathcal{M}_{\mathbf{k}, l, \mathbb{1}}(\Sigma, X, L, \mathbf{d})$, by

$$
\mathcal{B}=B_{\mathbf{k}, l, \mathbb{1}}^{1, p}(\Sigma, X, L, \mathbf{d}):=B^{1, p}(\Sigma, X, L, \mathbf{d}) \times \prod_{a}(\partial \Sigma)_{a}^{\left(k_{a}+\mathbb{k}_{a}\right)} \times \Sigma^{(l+\mathbb{1})} \backslash \triangle .
$$

If we use vectors $\vec{z}=\left(z_{a i}\right), \vec{w}=\left(w_{j}\right), \vec{p}=\left(p_{a i}\right), \vec{q}=\left(q_{\jmath}\right)$ to denote marked points respectively, then we denote elements of $B_{\mathbf{k}, l, \mathbb{1}}^{1, p}(\Sigma, X, L, \mathbf{d})$ by $\mathbf{u}=(u, \vec{z}, \vec{w}, \vec{p}, \vec{q})$. We simply denote the restricted pullback bundle $\left(\left.u\right|_{\partial \Sigma}\right)^{*} T L$ by $u^{*} T L$. By straightforward computations we have the following proposition.

Proposition 3.1 The linearization of (3.1) at $\mathbf{u} \in \mathcal{M}_{\mathbf{k}, l, \mathbb{1}}(\Sigma, X, L, \mathbf{d})$ is

$$
\begin{aligned}
D_{\mathbf{u}}:=D_{\mathbf{u}} \bar{\partial}_{(J, \nu)}: & \Gamma\left[(\Sigma, \partial \Sigma),\left(u^{*} T X, u^{*} T L\right)\right] \rightarrow \Omega^{0,1}\left(\Sigma, u^{*} T X\right) \\
D_{\mathbf{u}}(\xi)= & \frac{1}{2}\left[\nabla \xi+J \circ \nabla \xi \circ j+\nabla_{\xi} J \circ d u \circ j\right] \\
& +\frac{1}{2}[T(\xi, d u)+J T(\xi, d u \circ j)]-\nabla_{\xi} \nu
\end{aligned}
$$

where $\nabla$ is the pullbacked connection on $u^{*} T X$ and $T(\zeta, \eta)=\nabla_{\zeta} \eta-\nabla_{\eta} \zeta-[\zeta, \eta]$ is the torsion of $\nabla$.

Denote by

$$
\mathcal{D}_{u}(\xi)=\frac{1}{2}\left[\nabla \xi+J \circ \nabla \xi \circ j+\nabla_{\xi} J \circ d u \circ j\right]-\nabla_{\xi} \nu .
$$

We can similarly define the Banach space bundle $\mathcal{E} \rightarrow B_{\mathbf{k}, l, \mathbb{1}}^{1, p}(\Sigma, X, L, \mathbf{d})$ fiberwise by

$$
\mathcal{E}_{\mathbf{u}}:=L^{p}\left(\Sigma, \Omega^{0,1}\left(u^{*} T X\right)\right)
$$


So we still think

$$
\bar{\partial}_{(J, \nu)}: B_{\mathbf{k}, l, \mathbb{1}}^{1, p}(\Sigma, X, L, \mathbf{d}) \rightarrow \mathcal{E}
$$

as a section of $\mathcal{E}$. And we also denote by

$$
D:=D \bar{\partial}_{(J, \nu)}: T B_{\mathbf{k}, l, \mathbb{1}}^{1, p}(X, L, \mathbf{d}) \rightarrow \mathcal{E}
$$

the vertical component of the linearization of $\bar{\partial}_{(J, \nu)}$. From the Proposition 6.14 in $\left[\mathrm{L}\right.$ we know that for each $\mathbf{u} \in B_{\mathbf{k}, l, \mathbb{1}}^{1, p}(\Sigma, X, L, \mathbf{d})$, both $D_{\mathbf{u}}$ and $\mathcal{D}_{u}$ are Fredholm operators with the same index $\operatorname{ind}\left(D_{\mathbf{u}}\right)=\operatorname{ind}\left(\mathcal{D}_{u}\right)=\mu+n(1-g)$, where $\mu=$ $\mu\left(u^{*} T X, u^{*} T L\right)$ is the total boundary Maslov index associated with the vector bundle pair $\left(u^{*} T X, u^{*} T L\right)$ defined in the Appendix (A.1). In particular, if $u$ is a $(J, \nu)$-holomorphic map with closed genus $g$ domain curve, then $\operatorname{ind}\left(D_{\mathbf{u}}\right)=$ $\operatorname{ind}\left(\mathcal{D}_{u}\right)=2 c_{1}+2 n(1-g)$, where $c_{1}$ is the first Chern number of the bundle $u^{*} T X \rightarrow \Sigma$.

Also we suppose $\Sigma$ is biholomorphic to its conjugation $\bar{\Sigma}$, i.e. there exists an anti-holomorphic involution $c: \Sigma \rightarrow \Sigma$. Now for each $J \in \mathcal{J}_{\omega, \phi}$, we denote by $\mathcal{P}_{\phi, c}^{J}$ the set of $\nu \in \mathcal{P}$ satisfying $d \phi \circ \nu \circ d c=\nu$. Recall

$$
\mathbb{J}:=\left\{(J, \nu) \mid J \in \mathcal{J}_{\omega}, \nu \in \mathcal{P}\right\},
$$

and

$$
\mathbb{J}_{\phi}:=\left\{(J, \nu) \mid J \in \mathcal{J}_{\omega, \phi}, \nu \in \mathcal{P}_{\phi, c}^{J}\right\} \subset \mathbb{J} .
$$

We still assume $L$ is relatively $\operatorname{Pin}^{ \pm}$and fix a relative $\operatorname{Pin}^{ \pm}$structure $\mathfrak{P}$ on L. If $L$ is orientable, we fix an orientation on $L$.

Denote the orthogonal projection onto the normal bundle $N_{V}$ by $\xi \mapsto \xi^{N}$. Since $L_{V}$, if nonempty, is a submanifold of $L$, we denote the normal bundle of $L_{V}$ in $L$ by $N_{L_{V}}$. Then for each $(J, \nu)$-holomorphic open map $u$ whose image lies in $\left(V, L_{V}\right)$, the operator

$$
\begin{gathered}
D_{u}^{N}: \Gamma\left[(\Sigma, \partial \Sigma),\left(u^{*} N_{V}, u^{*} N_{L_{V}}\right)\right] \rightarrow \Omega^{0,1}\left(\Sigma, u^{*} N_{V}\right), \\
D_{\mathbf{u}}^{N}(\xi)=\left[D_{\mathbf{u}}(\xi)\right]^{N},
\end{gathered}
$$

restricting the vertical linearization of $\bar{\partial}_{(J, \nu)}$ at $u$ to the normal bundle, is also a real linear Fredholm operator.

Similar to the construction of relative GW invariants, we will restrict attention to a subspace of $\mathbb{J}$ (or $\mathbb{J}_{\phi}$ involving the involution ) consisting of pairs $(J, \nu)$ that are compatible with $V$ in the following sense. Recall we let $\pi_{i}, i=1,2$, denote the projection from $\Sigma \times X$ to the $i^{\text {th }}$ factor and let $\pi_{i}^{\prime}$ denote the restriction of $\pi_{i}$ to $\partial \Sigma \times L$.

Definition 3.1 We say the pair $(J, \nu) \in \mathbb{J}$ or $\mathbb{J}_{\phi}$ is $V$-compatible if the following three conditions hold:

(1) $J$ preserves $T V,\left.\nu^{N}\right|_{V}=0$, and if $L \cap V \neq \emptyset$, then $\left.\nu\right|_{\partial \Sigma \times L_{V} \times \mathcal{B}}$ carries a sub-bundle $\pi_{1}^{\prime *} T \partial \Sigma \subset \pi_{1}^{*} T \Sigma$ to the sub-bundle $\pi_{2}^{\prime *}\left(T L_{V}\right) \subset \pi_{2}^{*} T X$; 
and for all $\xi \in\left(N_{V}, N_{L_{V}}\right), v \in T V$ and $\vartheta \in T \Sigma$

(2) $\left[\left(\nabla_{\xi} J+J \nabla_{J \xi} J\right)(v)\right]^{N}=\left[\left(J \nabla_{J v} J\right) \xi+\left(\nabla_{v} J\right) \xi\right]^{N}$;

(3) $\left[\left(J \nabla_{\nu(\vartheta)} J\right) \xi\right]^{N}=\left[\left(\nabla_{\xi} \nu+J \nabla_{J \xi} \nu\right)(\vartheta)\right]^{N}$.

We denote by $\mathbb{J}^{V}\left(\right.$ resp. $\mathbb{J}_{\phi}^{V}$ ) the set of all $V$-compatible pairs $(J, \nu) \in \mathbb{J}$ (resp. $\left.(J, \nu) \in \mathbb{J}_{\phi}\right)$.

Remark. The definitions above are modification of the closed version of the Definition 3.2 of [IP1. However, in our open case, the condition (1), compared with the condition (a) of the Definition 3.2 of [IP1], involves the Lagrangian boundary condition (see (2.2) for the definition of inhomogeneous perturbation $\nu$ ). It implies that $V$ is a $J$-holomorphic submanifold, and that $(J, \nu)$-holomorphic (open) curves in $V$ or $\left(V, L_{V}\right)$ are also $(J, \nu)$-holomorphic in $X$ or $(X, L)$, moreover, $\bar{\partial}_{(J, \nu)}$ gives rise to elliptic boundary value problems for both two kinds of open $(J, \nu)$-maps into $(X, L)$ and $\left(V, L_{V}\right)$. When $L \cap V=\emptyset$, conditions (2) and (3) are the same as the conditions (b) and (c) of the Definition 3.2 of [IP1] (note $\xi \in\left(N_{V}, N_{L_{V}}\right)$ means the section of normal bundle $N_{V}$ takes value in $N_{L_{V}}$ over $L_{V}$, if nonempty). In fact, they are mainly used to assure the following Lemma 3.1 holds and the same conclusion of Lemma 3.1 is important for the discussion in [IP1], since they desire the boundary strata of codimension at least 2 .

Conditions (2) and (3) ensure that for each $(J, \nu)$-holomorphic map with closed domain curve whose image lies in $V$

$$
u: \Sigma \rightarrow V
$$

the operator

$$
\begin{gathered}
\mathcal{D}_{u}^{N}: \Gamma\left[\left(\Sigma, u^{*} N_{V}\right) \rightarrow \Omega^{0,1}\left(\Sigma, u^{*} N_{V}\right),\right. \\
\mathcal{D}_{u}^{N}(\xi)=\left[\mathcal{D}_{u}(\xi)\right]^{N}
\end{gathered}
$$

by restricting the linearization of $\bar{\partial}_{(J, \nu)}$ at $u$ to the normal bundle, is a complex linear operator, i.e. we have the following lemma which is taken from [IP1].

Lemma 3.1 Choose $(J, \nu) \in \mathbb{J}^{V}$ (resp. $\left.\mathbb{J}_{\phi}^{V}\right)$. Then for each $(J, \nu)$-holomorphic map u from closed domain curve whose image lies in $V$, the operator $\mathcal{D}_{u}^{N}$ is a complex linear operator.

In the following we will not distinguish $D$ and $\mathcal{D}$ and denote always by $D$ the linearization operator. In the end of the section, we show a local normal form for open holomorphic maps near the points where they intersect $V$. Here the argument is similar to the Lemma 3.4 in IP1.

Take a pair $(J, \nu)$ satisfying the condition (1) in the Definition 3.1, Let $V$ be a codimension two $J$-holomorphic submanifold of $X$, and $u:(\Sigma, \partial \Sigma) \rightarrow(X, L)$ be a $(J, \nu)$-holomorphic map which intersects $V$. Suppose $L_{V}=V \cap L \neq \emptyset$. Take a boundary marked point $p_{a \imath} \in(\partial \Sigma)_{a}$ and an interior marked point $q_{\jmath}$ satisfying 
$\mathbb{p}=u\left(p_{a \imath}\right) \in L_{V}$ and $\mathbb{q}=u\left(q_{\jmath}\right) \in V$. Let $\mathbb{H}=\{z=x+\mathrm{i} y \mid y \geq 0\}$ be the upper half complex plane. Fix a local holomorphic coordinate $z \in \mathbb{H}$ on a half open disc $\mathfrak{D}$ in $\Sigma$ containing $p_{a \imath}$ or a local holomorphic coordinate $z^{\prime} \in \mathbb{C}$ on an open disc $\mathcal{O}$ in $\Sigma$ containing $q_{\text {J }}$. Also fix local coordinates $\left\{v^{i}\right\}=\left\{v^{i_{1}}, v^{i_{2}}\right\}\left(1 \leq i_{1} \leq n-1\right.$, $\left.n \leq i_{2} \leq 2 n-2\right)$ in an open set $\mathscr{O}_{V}$ in $V$ such that $\left\{v^{i_{1}}\right\}$ are the local coordinates in the open set $\mathscr{O}_{V} \cap L_{V}$ in $L_{V}$. And we extend $\left\{v^{i}\right\}$ to local coordinates $\left\{v^{i}, x\right\}$ for $X$ with $x \equiv 0$ along $V$ and so that $x=x^{1}+\mathrm{i} x^{2}$ with $J\left(\frac{\partial}{\partial x^{1}}\right)=\frac{\partial}{\partial x^{2}}$ and $J\left(\frac{\partial}{\partial x^{2}}\right)=-\frac{\partial}{\partial x^{1}}$, moreover, we require that $\left\{v^{i_{1}}, x^{1}\right\}$ are just local coordinates for $L$.

Lemma 3.2 (normal form). Suppose that $\Sigma$ is a smooth connected Riemann surface with boundary and $u$ is a $(J, \nu)$-holomorphic map which intersects $V$ at $\mathbb{p}$ or $\mathbb{q}$. Then either (1) $u(\Sigma) \subset V$, or (2) there exist an integer $K>0$ (depending on $\mathbb{P}$ or $\mathbb{q})$ and a nonzero $a_{0} \in \mathbb{C}$ such that near interior intersection point

$$
u\left(z^{\prime}, \overline{z^{\prime}}\right)=\left(q^{i}+O\left(\left|z^{\prime}\right|\right), a_{0} z^{K}+O\left(\left|z^{\prime}\right|{ }^{K+1}\right)\right),
$$

or near boundary intersection point

$$
u(z, \bar{z})=\left(\mathbb{p}^{i}+O(|z|), c_{0} z^{K}+O\left(|z|^{K+1}\right)\right),
$$

in the local coordinates $z^{\prime}$ or $z$ and $\left\{v^{i}, x\right\}$, respectively, where $O\left(|z|^{k}\right)$ denotes a function of $z$ and $\bar{z}$ that vanished to order $k$ at $z=0$. In particular, writing $z=r+\sqrt{-1} s$ and restricting (3.8) to the real part, we have

$$
\left.u(z, \bar{z})\right|_{\mathbb{R}}=u(r)=\left(\mathbb{p}^{i}+O(r), c_{0} r^{K}+O\left(r^{K+1}\right)\right),
$$

in the local coordinates $\left\{v^{i_{1}}, x^{1}\right\}$ in $L$, where $c_{0}$ is a nonzero real number.

Proof. When we consider an interior intersection point $\mathbb{q}$, the argument is the same as the one in Lemma 3.4 of [IP1]. When we consider a boundary intersection point $\mathbb{p}$, the argument can not apply directly, since the map $u$ is only continuous at the boundary. Fortunately, this is just a local problem, the above similar conclusion still holds. Indeed, under the local coordinates $\left(v^{i}, x\right)$ around $\mathbb{p}$ and $z \in A \subset \mathbb{H}$ around $p_{a \imath}$, every $v^{i}$ or $x$ is a function : $A \rightarrow B \subset \mathbb{C}$ which is only continuous on $A \cap\{\operatorname{Im} z=0\}$. Since the map $u$ is locally holomorphic, by definition, each $v^{i}$ (or $x$ ) can be extended to a holomorphic function $v_{\mathbb{C}}^{i}$ (or $x_{\mathbb{C}}$ ) $: U \rightarrow \mathbb{C}$, where $U$ is an open neighborhood of $A$ in $\mathbb{C}$. Then the argument in [IP1] can go through for $u_{\mathbb{C}}$ and obtain the formula (3.8) by restricting to $\mathbb{H}$.

\section{Moduli space of $V$-regular and $V$-stable maps}

In the rest of the paper, we only consider the special case that $L \cap V=\emptyset$. Also we assume that all moduli spaces are generically irreducible, that is they generically contain no multiply covered maps which, for instance, can be verified by the imposed semi-positivity conditions on $(X, L)$ and $V$. 


\subsection{V-regular open stable maps}

Definition 4.1 Given a codimension two symplectic submanifold $V$ of $(X, \omega)$. $A$ stable $(J, \nu)$-holomorphic map $u:(\Sigma, \partial \Sigma) \rightarrow(X, L)$ is called $V$-regular if no component of its domain is mapped entirely into $V$ and if neither any marked point nor any double point is mapped into $V$.

The set of $V$-regular open maps forms an open subset of the space of open stable maps, denote it by $\mathcal{M}^{V}(\Sigma, X, L, \mathbf{d})$. Denote by $\mathcal{M}_{\mathbf{k}, l, \mathbb{1}}^{V}(\Sigma, X, L, \mathbf{d})$ the space of $V$-regular maps with marked points.

We denote by $\Phi=\left(s_{1}, \cdots, s_{\mathbb{1}}\right)$ the list of multiplicities of interior intersection points, where $s_{j}$ are positive integer numbers. And we define the degree, length, and order of $\$$ by

$$
\operatorname{deg} \mathbb{\$}:=\sum_{j=1}^{\mathbb{1}} s_{j}, \quad \operatorname{length}(\mathbb{\$}):=\mathbb{1}, \quad \operatorname{ord}(\mathbb{\$}):=|\mathbb{\$}|=\prod_{j=1}^{\mathbb{1}} s_{j} .
$$

The vector $\$$ labels the component of $\mathcal{M}_{\mathbf{k}, l, \mathbb{1}}^{V}(\Sigma, X, L, \mathbf{d})$. We denote each $\$$-labeled component of $\mathcal{M}_{\mathbf{k}, l, \mathbb{1}}^{V}(\Sigma, X, L, \mathbf{d})$ by

$$
\mathcal{M}_{\mathbf{k}, l, \mathbb{1}}^{V, \mathbb{1}}(\Sigma, X, L, \mathbf{d}) \subset \overline{\mathcal{M}}_{\mathbf{k}, l+\mathbb{1}}(\Sigma, X, L, \mathbf{d}) .
$$

Forgetting the additional $\mathbb{1}$ marked points defines a projection

$$
\begin{gathered}
\mathcal{M}_{\mathbf{k}, l, \mathbb{1}}^{V, \mathbb{s}}(\Sigma, X, L, \mathbf{d}) \\
\downarrow \\
\mathcal{M}_{\mathbf{k}, l}^{V}(\Sigma, X, L, \mathbf{d})
\end{gathered}
$$

onto one component of $\mathcal{M}_{\mathbf{k}, l}^{V}(\Sigma, X, L, \mathbf{d})$, which is the disjoint union of such components. So for each fixed $\mathbf{S}$, (4.1) is a covering map to its image whose deck transformation group is the the group of renumberings of the $\mathbb{1}$ interior marked points.

For any integer $m \geq 1$, let $\mathbb{J}^{V, m}$ be the completion of the space $\mathbb{J}^{V}$ of all smooth pairs $(J, \nu)$ in the $C^{m}$-topology. Then $\mathbb{J}^{V, m}$ is a $C^{m}$-smooth Banach manifold. Denote by $B_{\mathbf{k}, l}^{*, V, 1, p}(\Sigma, X, L, \mathbf{d})$ the irreducible part of ambient Sobolev space of $V$ regular maps, which is also a Banach manifold. Let $\mathfrak{M}_{\mathbf{k}, l}^{V}=B_{\mathbf{k}, l}^{*, V, 1, p}(\Sigma, X, L, \mathbf{d}) \times$ $\mathbb{J}^{V, m}$, then we define the following universal moduli space

$\mathcal{U} \mathcal{M}_{\mathbf{k}, l}^{V}=\mathcal{U} \mathcal{M}_{\mathbf{k}, l}^{V}(\Sigma, X, L, \mathbf{d}):=\left\{(\mathbf{u},(J, \nu)) \mid(J, \nu) \in \mathbb{J}^{V, m}, \mathbf{u} \in \mathcal{M}_{\mathbf{k}, l}^{V}(\Sigma, X, L, \mathbf{d})\right\}$

and its irreducible part

$$
\mathcal{U} \mathcal{M}_{\mathbf{k}, l}^{* V}=\left\{(u, \vec{z}, \vec{w}, J, \nu) \in \mathfrak{M}_{\mathbf{k}, l}^{V} \mid \bar{\partial}_{J, \nu} u=0\right\} .
$$

The irreducible part of the universal moduli space involving the intersection data is denoted by $\mathcal{U} \mathcal{M}_{\mathbf{k}, l, \mathbb{1}}^{*}$. When $p>2$, by elliptic regularity, each element $u$ of $\mathcal{U} \mathcal{M}_{\mathbf{k}, l}^{* V}$ is smooth. 
Proposition 4.1 The irreducible universal moduli space $\mathcal{U} \mathcal{M}_{\mathbf{k}, l}^{* V}$ is a smooth $B a$ nach submanifold of $\mathfrak{M}_{\mathbf{k}, l}^{V}$.

Proof. There is an infinite dimensional vector bundle

$$
\mathcal{E} \rightarrow \mathfrak{M}_{\mathbf{k}, l}^{V},
$$

with each fiber $\mathcal{E}_{(\mathbf{u}, J, \nu)}:=L^{p}\left(\Sigma, \Omega^{0,1}\left(u^{*} T X\right)\right)$. The $(J, \nu)$-holomorphic equation defines a section of this bundle by

$$
\mathcal{S}: \mathfrak{M}_{\mathbf{k}, l}^{V} \rightarrow \mathcal{E}, \quad \mathcal{S}(\mathbf{u}, J, \nu)(x)=\bar{\partial}_{J} u(x)-\nu(u(x)) .
$$

Since $\mathcal{U} \mathcal{M}_{\mathbf{k}, l}^{* V}=\mathcal{S}^{-1}(0)$, one has to show that $\mathcal{S}$ is transverse to the zero section. Let $\mathcal{S}(\mathbf{u}, J, \nu)=0$. We have

$$
\begin{gathered}
T_{\mathbf{u}} B_{\mathbf{k}, l}^{*, V, 1, p}(\Sigma, X, L, \mathbf{d})=W^{V, 1, p}\left(\Omega^{0}\left(u^{*} T X, u^{*} T L\right)\right) \\
T_{(J, \nu)} \mathbb{J}^{V, m}=C^{m}(\operatorname{End}(T X, J)) \oplus C^{m}\left(\operatorname{Hom}_{J}(T \Sigma, T X)\right)
\end{gathered}
$$

where $\operatorname{End}(T X, J)=\{A \mid A: T X \rightarrow T X, A J+J A=0\}$. Recall that $\operatorname{Hom}_{J}(T \Sigma, T X)$ is the space of anti- $J$-linear homomorphism with respect to the complex structure. There is an identification

$$
\left.\operatorname{Hom}_{J}(T \Sigma, T X)\right|_{\Gamma_{u}}=\Omega_{J}^{0,1}\left(u^{*} T X\right),
$$

where $\Gamma_{u} \subset \Sigma \times X$ is the graph of $u$.

Now we consider the vertical differential

$$
\begin{array}{r}
D \mathcal{S}(\mathbf{u}, J, \nu): W^{V, 1, p}\left(\Omega^{0}\left(u^{*} T X, u^{*} T L\right)\right) \oplus C^{m}(\operatorname{End}(T X, J)) \\
\oplus C^{m}\left(\operatorname{Hom}_{J}(T \Sigma, T X)\right) \longrightarrow L^{p}\left(\Sigma, \Omega^{0,1}\left(u^{*} T X\right)\right) .
\end{array}
$$

Then we have

$$
D \mathcal{S}(\mathbf{u}, J, \nu)(\xi, A, \mu)=D_{u} \xi+\frac{1}{2} u^{*} A \circ d u \circ j_{\Sigma}-\left.\mu\right|_{\Gamma_{u}},
$$

where

$$
D_{\mathbf{u}}:=D_{\mathbf{u}} \bar{\partial}_{(J, \nu)}: W^{V, 1, p}\left(\Omega^{0}\left(u^{*} T X, u^{*} T L\right)\right) \rightarrow L^{p}\left(\Omega^{0,1}\left(u^{*} T X\right)\right) .
$$

By elliptic regularity theory, for any $p>2, u$ is in the space $C^{m}$ if $(J, \nu)$ is in $C^{m}$. Moreover, the cokernel of $D_{\mathbf{u}}$ is contained in $C^{m}\left(\Omega^{0,1}\left(u^{*} T X\right)\right)$. Since $D_{\mathbf{u}}$ is Fredholm, its cokernel is of finite dimension. On the other hand, considering the definition of $\nu$ (2.2) and the identification (4.3), the map

$$
\left.\mu\right|_{\Gamma_{u}}: C^{m}\left(\operatorname{Hom}_{J}(T \Sigma, T X)\right) \longrightarrow C^{m}\left(\Omega^{0,1}\left(u^{*} T X\right)\right)
$$

is surjective. Therfore, by (4.5), $D \mathcal{S}(\mathbf{u}, J, \nu)$ is surjective. Thus, the implicit function theorem implies that the universal moduli space $\mathcal{U} \mathcal{M}_{\mathbf{k}, l}^{* V}$ is a smooth Banach submanifold of $\mathfrak{M}_{\mathbf{k}, l}^{V} \cdot \square$ 
Lemma 4.1 Fix a conformal structure $j$ on $\Sigma$. For generic $V$-compatible pair $(J, \nu) \in \mathbb{J}^{V}$, the irreducible part of $\mathcal{M}_{\mathbf{k}, l, \mathbb{1}}^{V, \mathbb{s}}(\Sigma, X, L, \mathbf{d})$ is an orbifold with boundary of dimension

$$
\operatorname{dim} \mathcal{M}_{\mathbf{k}, l, \mathbb{1}}^{* V, \mathbb{s}}(\Sigma, X, L, \mathbf{d})=\mu(d)+n(1-g)+k+2(l+\mathbb{1}-\operatorname{deg} \$)-\operatorname{dim} A u t(\Sigma) .
$$

Proof. The argument will be a slight modification of the ones used in the Lemma 4.2 and Lemma 4.3 of [IP1], since no boundary points contact $V$. The main points are as follows.

Similar to the Proposition 17.1 in FOOO for special case $g=0$ and $l=0$, and the Theorem C.10 in [McS], it is easy to calculate the dimension of $\mathcal{M}_{\mathbf{k}, l+\mathbb{1}}^{*}$ which is

$$
\operatorname{dim} \mathcal{M}_{\mathbf{k}, l+\mathbb{1}}^{* V}(\Sigma, X, L, \mathbf{d})=\mu(d)+n(1-g)+k+2(l+\mathbb{1})-\operatorname{dim} A u t(\Sigma) .
$$

If we show that the irreducible part of universal moduli space $\mathcal{U} \mathcal{M}_{\mathbf{k}, l, \mathbb{1}}^{* V, \mathbb{s}}$ is an orbifold, then the Sard-Smale transversality theorem implies that for generic $(J, \nu) \in \mathbb{J}^{V, m}$ the irreducible part of the $V$-regular moduli space is an orbifold.

The Proposition 4.1 shows that $\mathcal{U M}_{\mathbf{k}, l}^{* V}$ is a smooth Banach orbifold. Then the point is to show that the contact condition corresponding to each sequence $\mathbb{S}$ is transverse. That will imply that the irreducible universal moduli space $\mathcal{U} \mathcal{M}_{\mathbf{k}, l, \mathbb{1}}^{*} V$ is an orbifold and the fomula (4.7) holds. From the proof of Lemma 4.2 and Lemma 4.3 of [IP1], we know that the transverality of contact condition arises from the local analysis around those intersecting points with $V$. Geometrically, that means the $V$-compatibility conditions assure that local variations of the inhomogeneous perturbation and the $V$-regular maps would realize other intersecting multiplicities with given degree. Since we assume that $L \cap V=\emptyset$ and use the similar $V$-compatibility conditions as ones in [IP1], the similar argument can go through.

\subsection{Limits of $V$-regular open maps}

We come to construct a compactification of each component of the moduli space of $V$-regular open maps. To compactify $\mathcal{M}_{\mathbf{k}, l, \mathbb{1}}^{V, \mathbf{s}}(\Sigma, X, L, \mathbf{d})$, we take its closure

$$
\mathcal{C} \mathcal{M}_{\mathbf{k}, l, \mathbb{1}}^{V, \mathbb{\Phi}}(\Sigma, X, L, \mathbf{d})
$$

in the stable moduli space $\overline{\mathcal{M}}_{\mathbf{k}, l+\mathbb{1}}(\Sigma, X, L, \mathbf{d})$. In fact, the closure lies in the subset of $\overline{\mathcal{M}}_{\mathbf{k}, l+\mathbb{1}}(\Sigma, X, L, \mathbf{d})$ consisting of open stable maps whose last $\mathbb{1}$ marked points are mapped into $V$, still with associated multiplicities $\mathbb{s}$, although the actual order of contact might be infinite.

We will show that the closure is an orbifold with boundary. That is to prove that the frontier $\mathcal{C} \mathcal{M}^{V} \backslash \mathcal{M}^{V}$ is a subset of codimension at least 1. Since such frontier is a subset of the space of stable maps, it is stratified according to the type of bubble structure of the domain. The following proposition is the main result in this section describing the structure of the closure $\mathcal{C M}^{V}$. 
Proposition 4.2 For generic pair $(J, \nu) \in \mathbb{J}^{V}$, each stratum of the irreducible part of

$$
\mathcal{C} \mathcal{M}_{\mathbf{k}, l, \mathbb{1}}^{V, \mathbb{s}}(\Sigma, X, L, \mathbf{d}) \backslash \mathcal{M}_{\mathbf{k}, l, \mathbb{1}}^{V, \mathbb{s}}(\Sigma, X, L, \mathbf{d})
$$

is an orbifold of dimension at least one less than the dimension (4.7) of $\mathcal{M}_{\mathbf{k}, l, \mathbb{1}}^{*} V(\Sigma, X, L, \mathbf{d})$.

We follow the way of [IP1, which studies moduli spaces of closed $(J, \nu)$-maps according to the different types of limits, to prove this proposition for two basic cases:

Case 1. stable maps with no components or special ((k,l)-marked and double) points lying entirely in $V$;

Case 2. stable maps with some components in $V$ and some off $V$.

The full proof of this proposition will finish in the Theorem 4.1

Case 1. Since we do not admit the degeneration of domain, each stratum of this type is labeled by the pair of positive integer numbers $(\mathfrak{b}, \dot{i})$ of boundary and interior double points of their nodal domain curve $\widehat{\Sigma}$. For such fixed $\widehat{\Sigma}$, the corresponding stratum is $\mathcal{M}_{\mathbf{k}, l, \mathbb{1}}^{V, \mathbb{1}}(\widehat{\Sigma}, X, L, \mathbf{d})$.

Lemma 4.2 In the 'Case 1', for generic pair $(J, \nu) \in \mathbb{J}^{V}$, the irreducible part of the stratum $\mathcal{M}_{\mathbf{k}, l, \mathbb{1}}^{V, \mathbb{s}}(\widehat{\Sigma}, X, L, \mathbf{d})$ of $\mathcal{C M}^{V}$ is an orbifold of dimension $\mathfrak{b}+2 \dot{\mathrm{i}}$ less than (4.7).

Proof. The argument is standard and is open version parallel to the ones in [IP1]. Note that the dimension argument is direct since the degree of freedom of $z_{i} \in \partial \Sigma$ is one.

Case 2. Now we consider the case that there is a sequence of $V$-regular open maps converges to a limit map $u \in \mathcal{C M}_{\mathbf{k}, l, \mathbb{1}}^{V, \mathbb{1}}(\Sigma, X, L, \mathbf{d})$ whose domain is the union $\Sigma=\Sigma_{1} \cup \Sigma_{2}$, where $\Sigma_{1}$ is either a bubble domain or a union of several unconnected bubble domains of (total) genus $g_{1}$ (for simplicity and without loss of generality, we only consider the one bubble domain case), $\Sigma_{2}$ is a bubble domains of genu $g_{2}, \partial \Sigma_{2}=\emptyset$, and $u$ restricts to a $V$-regular map $u_{1}: \Sigma_{1} \rightarrow X$ and a holomorphic map $u_{2}: \Sigma_{2} \rightarrow V$ into $V$. Limit maps of this type arise from sequences of $V$-regular maps in which either

(1) two of the $\mathbb{1}$-contact intersection points collide in the domain, or

(2) one of the original $l$-marked points whose image sinks into $V$, collides with a contact point.

In either case the collision produces a ghost bubble map $u_{2}: \Sigma_{2} \rightarrow V$ whose energy is at least $\hbar_{V}$ by Lemma 2.1 .

Then note that $u_{1}^{-1}(V)$ consists of the nodal points $\Sigma_{1} \cap \Sigma_{2}$ and some of the last $\mathbb{1}$ marked points $q_{j} \in \Sigma$. The nodes are defined by identifying points $x_{j} \in \Sigma_{1}$ and $y_{j} \in \Sigma_{2}$. Since $u_{1}$ is $V$-regular and $u_{1}\left(x_{j}\right) \in V$, then Lemma 3.2 associates a multiplicity $s_{j}^{\prime}$ to each $x_{j}$, the multiplicity vector is denoted by

$$
\mathbb{s}^{\prime}=\left(s_{1}^{\prime}, s_{2}^{\prime}, \cdots\right) .
$$


For convenience, images of these nodes $x_{j}$ and $y_{j}$ is denoted by vectors $\mathbb{1}^{\prime}$. Similarly, since $u$ arises as a limit of $V$-regular maps, the $q_{\jmath}$, which are limits of the contact points with $V$, also have associated multiplicities. We split the set of $q_{\text {J }}$ into the points $\left\{q_{j}^{1}\right\}$ on $\Sigma_{1}$ and $\left\{q_{j}^{2}\right\}$ on $\Sigma_{2}$, denote by

$$
\mathbb{s}^{1}=\left(s_{1}^{1}, s_{2}^{1}, \cdots\right) \quad \text { and } \quad \mathbb{s}^{2}=\left(s_{1}^{2}, s_{2}^{2}, \cdots\right)
$$

the associated multiplicity vectors. Hence we write $u$ as a pair

$$
\left(u_{1}, u_{2}\right) \in \mathcal{M}_{g_{1}, \mathbf{k}_{1}, l_{1}, \mathbb{1}_{1}+\mathbb{I}^{\prime}}^{V,,^{1}+\mathbb{s}^{\prime}}\left(\Sigma_{1}, X, L, \mathbf{d}_{1}\right) \times \overline{\mathcal{M}}_{g_{2}, \mathbf{k}_{2}, l+\mathbb{1}_{2}+\mathbb{I}^{\prime}}\left(\Sigma_{2}, V, L_{V}, \mathbf{d}_{2}\right)
$$

where $\mathbf{d}_{i}=\left[u_{i}\right]$ with $\mathbf{d}_{1}+\mathbf{d}_{2}=\mathbf{d}$ satisfying the matching conditions $u_{1}\left(x_{j}\right)=$ $u_{2}\left(y_{j}\right)$, and $\left(\mathbf{k}_{1}, l_{1}\right)+\left(\mathbf{k}_{2}, l_{2}\right)=(\mathbf{k}, l)$.

Lemma 4.3 In this case 2, the only elements (4.9) lying in $\mathcal{C} M^{V, s}$ are those for which there exists a (singular) section $\xi \in \Gamma\left((\Sigma, \partial \Sigma),\left(u_{2}^{*} N_{V}, u_{2}^{*} N_{L_{V}}\right)\right)$ nontrivial on at least one component of $\Sigma_{2}$ with zeros of order $s_{j}^{2}$ at $q_{j}^{2} \in \Sigma$, poles of order $s_{j}^{\prime}$ at $y_{j}$, and $D_{u_{2}}^{N} \xi=0$ where $D_{u_{2}}^{N} \xi$ is as in (3.5).

Proof. It is an open version parallel to the delicate renormalization argument used in proposition 6.6 of [IP1]. The renormalization will be done in a projective compactification $\mathbb{P}\left(\left(N_{V}, N_{L_{V}}\right) \oplus(\mathbb{C}, \mathbb{R})\right)$ of the normal bundle. By applying the renormalization argument we see that it can still work well in our open case. We will not repeat the discussion.

\subsection{The space of $V$-stable open maps}

From last subsection we see that the limit of a sequence of $V$-regular open maps is a stable map whose components are of the basic types described in Case 1 and 2. Actually, the components of the limit map are also partially ordered according to the rate at which they sink into $V$. In this section we will make this precise by extending the concept layer structure, which is originally introduced in IP1 for closed domain curves, to our domain of open Riemann surfaces. And then we construct a compactification of the space of $V$-regular maps. Let $\Sigma$ be a stable curve with boundary.

Definition 4.2 A layer structure on $\Sigma$ is the assignment of a non-negative integer $\lambda_{j}$ to each irreducible component $\Sigma_{j}$ to $\Sigma$, such that at least one component must have $\lambda_{j}=0$ or 1 .

The union of all the components with $\lambda_{j}=K$ is called the layer $K$ part of $\Sigma$, denoted by $\Lambda_{K}$. Note that $\Lambda_{K}$ might not be a connected.

Definition 4.3 A marked layer structure on a $(\mathbf{k}, l+\mathbb{1})$-marked bordered bubble surface $\Sigma_{\mathbf{k}, l+\mathbb{1}}$ is a layer structure on $\Sigma$ together with

(1) a vector $\$$ recording the multiplicities of the last $\mathbb{1}$ interior intersection marked points, and

(2) a pair of vectors $(\alpha, \beta)$ which assigns multiplicities respectively to each boundary double point of $\partial \Lambda_{K} \cap \partial \Lambda_{L}$, and to each interior double point of $\Lambda_{K} \cap \Lambda_{L}$, $K \neq L$. 
Note that double points within a layer are not assigned a multiplicity.

Each layer $\Lambda_{K}$ then has interior points $q_{K, \jmath}$ of the type (1) with multiplicity vector $\$_{K}=\left(s_{K, j}\right)$, and has double pints with multiplicities. The double points in $\Lambda_{K}$ that are assigned multiplicities are divided into two types.

Let $\mathbb{\alpha}_{K}^{+}$(resp. $\beta_{K}^{+}$) be the vector derived from $\propto$ (resp. $\beta$ ) that gives the multiplicities of the boundary (resp. interior) double points $y_{K, i}^{+}$where $\Lambda_{K}$ meets the higher layers at boundary (resp. interior part), i.e. the points $\partial \Lambda_{K} \cap \partial \Sigma_{j}$ (resp. $\Lambda_{K} \cap \Sigma_{j}$ ) with $\lambda_{j}>K$. Let $\mathbb{\alpha}_{K}^{-}$(resp. $\beta_{K}^{-}$) be the similar vector of multiplicities of boundary (resp. interior) double points $y_{K, i}^{-}$where $\Lambda_{K}$ meets the lower layers.

Then we associate operators $D_{K}^{N}$ similar to (3.5) defined on the layers $\Lambda_{K}$, $K \geq 1$, as follows. For the interior double points, the argument is the same as the one in [IP1, so we only consider the special case that we assume there are only boundary double points. For each choice of $\mathbb{\alpha}=\mathbb{\alpha}_{K}^{-}=\left\{\alpha_{K, i}^{-}\right\}$and $\rho$, fix smooth weighting function $W, \rho$ which has the form $\left|z_{i}\right|^{\rho+\alpha_{K, i}^{-}}$in some local coordinates $z_{i}$ in a small half-disc centered at $y_{K, i}^{-}$and has no other zeros. Then given a stable map $u: \Lambda_{K} \rightarrow V$, denote by $L_{\bar{K}, \delta}^{m},\left(u^{*} N_{V}, u^{*} N_{L_{V}}\right)$ the Hilbert space of all $L_{l o c}^{m}$ sections of $\left(u^{*} N_{V}, u^{*} N_{L_{V}}\right)$ over $\left(\Lambda_{K}, \partial \Lambda_{K}\right) \backslash\left\{y_{K, i}^{-}\right\}$which are finite in the norm

$$
\|\xi\|_{m, \delta}^{2}=\sum_{l=1}^{m} \int_{\Lambda_{K}}\left|W_{, l+\delta} \cdot \nabla^{l} \xi\right|^{2} .
$$

For large $m$ the elements $\xi$ in this space have poles with $|\xi| \leq c\left|z_{i}\right|^{-\alpha_{K, i}^{-}-\delta}$ at each $y_{K, i}^{-}$and have $m-1$ continuous derivatives elsewhere on $\Lambda_{K}$. For such $m$, denote by $L_{K, \delta}^{m}\left(u^{*} N_{V}, u^{*} N_{L_{V}}\right)$ the closed subspace of $L_{-}^{m}, \delta\left(u^{*} N_{V}, u^{*} N_{L_{V}}\right)$ consisting of all sections that vanish to order $r_{K, j}$ and $s_{K, j}$ at $p_{K, j}$ and $q_{K, j}$ and order $\alpha_{K, i}^{+}$ at $y_{K, i}^{+}$. Hence by elliptic theory for weighted norms, the operator $D^{N}$ defines a bounded operator

$$
D_{K}^{N}: L_{K, \delta}^{m}\left(\left(\Lambda_{K}, \partial \Lambda_{K}\right),\left(u^{*} N_{V}, u^{*} N_{L_{V}}\right)\right) \rightarrow L_{K, \delta+1}^{m-1}\left(T^{*} \Lambda_{K} \otimes u^{*} N_{V}\right) .
$$

For generic $0<\delta<1, D_{K}^{N}$ is Fredholm with

$$
\begin{aligned}
\text { index } D_{K}^{N}= & \mu_{N_{V}}\left(\left[u\left(\Lambda_{K}\right)\right]\right)+\chi\left(\Lambda_{K}\right)+\operatorname{deg} \mathbb{\alpha}_{K}^{-}-\operatorname{deg} \mathbb{\alpha}_{K}^{+} \\
& +2\left(\operatorname{deg} \beta_{K}^{-}-\operatorname{deg} \Phi_{K}-\operatorname{deg} \mathbb{\beta}_{K}^{+}\right) \\
= & \chi\left(\Lambda_{K}\right)
\end{aligned}
$$

where $\chi\left(\Lambda_{K}\right)$ is the Euler characteristic of $\Lambda_{K}$, and the index formula would be more general for cases admitting interior double points. The formula is derived from the fact that the Euler class of the pair of complex line bundle and totally real sub-bundle $\left(u^{*} N_{V}, u^{*} N_{L_{V}}\right)$ can be computed from the zeros and poles of a section.

Then under the assumption $L \cap V=\emptyset$, we give the following definition of the stable maps we want. 
Definition 4.4 AV-stable map is a stable map $u \in \overline{\mathcal{M}}_{\mathbf{k}, l+\mathbb{1}}(\Sigma, X, L, \mathbf{d})$ together with

(1) a marked layer structure on its domain $\Sigma$ with $\left.u\right|_{\Lambda_{0}}$ being $V$-regular, and

(2) for each $K \geq 1$, an element $\xi_{K} \in \operatorname{ker} D_{K}^{N}$ defined on the layer $\Lambda_{K}$ that is a section nontrivial on every irreducible component of $\Lambda_{K}$.

Denote by $\overline{\mathcal{M}}_{\mathbf{k}, l, \mathbb{1}}^{V, \mathbf{s}}(\Sigma, X, L, \mathbf{d})$ the $\$$-labeled component of the set of all $V$ stable open maps. This contains the set $\mathcal{M}_{\mathbf{k}, l, \mathbb{1}}^{V, \mathbf{s}}(\Sigma, X, L, \mathbf{d})$ of $V$-regular maps as the open subset, i.e. the $V$-stable maps whose entire domain lies in layer 0 . Forgetting the data $\xi_{K}$ defines a map

$$
\mathscr{F}: \overline{\mathcal{M}}_{\mathbf{k}, l, \mathbb{1}}^{V, \mathbb{s}}(\Sigma, X, L, \mathbf{d}) \rightarrow \overline{\mathcal{M}}_{\mathbf{k}, l+\mathbb{1}}(\Sigma, X, L, \mathbf{d})
$$

Note that the number of layers of a $V$-stable map must be finite. Assume there are altogether $r+1$ layers $\Lambda_{0}, \Lambda_{1}, \cdots, \Lambda_{r}$. Each $V$-stable map $\left(\mathbf{u}, \xi_{1}, \cdots, \xi_{r}\right)$ determines an element of the space 8

$$
\mathcal{H}_{(X, L)}^{V}=H_{2}(X, L) \times \bigsqcup_{\$} V_{\Phi}
$$

as follows, where $V_{\mathbb{\Phi}}$ is the space, diffeomorphic to $V^{\mathbb{1}(\$)}$, of all sets of contact intersection points with multiplicities.

For sufficiently small $\varepsilon$, we can push the components in $V$ off $V$ by composing $u$ with $\exp \left(\varepsilon^{K} \xi_{K}\right)$ and for each $K$, smoothing the domain at the nodes $\Lambda_{K} \cap$ $\left(\cup_{L>K} \Lambda_{L}\right)$ and smoothly joining the images where the zeros of $\varepsilon^{K} \xi_{K}$ on $\Lambda_{K}$ approximate the poles of $\varepsilon^{K+1} \xi_{K+1}$. The resulting map

$$
u_{\xi}=\left.u\right|_{\Lambda_{0}} \# \exp \left(\varepsilon \xi_{1}\right) \# \cdots \# \exp \left(\varepsilon^{r} \xi_{r}\right)
$$

is $V$-regular and represents an element or a class in $\mathcal{H}_{(X, L)}^{V}$. Note that this class is independent of the choice of the small $\varepsilon$, and depends on each $\xi_{K}$ up to a nonzero multiplier.

Thus, we can associate a well-defined map

$$
\mathscr{H}: \overline{\mathcal{M}}_{\mathbf{k}, l, \mathbb{1}}^{V, \mathbf{1}}(\Sigma, X, L, \mathbf{d}) \rightarrow \mathcal{H}_{(X, L), \mathbf{d}}^{V, \mathbb{s}} .
$$

The following proposition is an open version similar to the Proposition 7.3 in [IP1.

Proposition 4.3 There exists a topology on $\overline{\mathcal{M}}_{\mathbf{k}, l, \mathbb{1}}^{V, \mathbb{s}}(\Sigma, X, L, \mathbf{d})$ such that this space of $V$-stable maps is compact and the maps $\mathscr{F}$ of 4.12) and $\mathscr{H}$ of 4.13) are continuous and differential on each stratum.

\footnotetext{
${ }^{8}$ Here, the space $\mathcal{H}_{(X, L)}^{V}$ is not really a parallel generalization of the space $\mathcal{H}_{X}^{V}$ appeared in (5.8) of [P1]. Our space $\mathcal{H}_{(X, L)}^{V}$ is less delicate than the space used in IP1. However, if $L \cap V=\emptyset$, we can also define the space of homology-intersection data as a covering map of $H_{2}(X, L) \times \bigsqcup_{\mathbb{s}} V_{\mathbf{s}}$, that will just be the case we consider to define relatively open invariants in this article.
} 
Proof. The argument is parallel to the one in Proposition 7.3 of [IP1. The idea is to analyse a sequence of $V$-regular maps and a more general sequence of $V$-stable maps to define the topology on $\overline{\mathcal{M}}_{\mathbf{k}, l, \mathbb{1}}^{V, \mathbb{s}}(\Sigma, X, L, \mathbf{d})$. The modifications are that we would use the bubble convergence Theorem 2.1 to our open case, and that the argument of open version renormalization which is simply described in the proof of lemma 4.3 will define the structure of a $V$-stable map on the limit map.

The next theorem is the key result needed to define the relative invariants, which implies the Proposition 4.2 ,

Theorem 4.1 The space of $V$-stable maps is compact and there exists a continuous map

$$
\varepsilon_{V}: \overline{\mathcal{M}}_{\mathbf{k}, l, \mathbb{1}}^{V, \mathbf{s}}(\Sigma, X, L, \mathbf{d}) \stackrel{\text { ev } \times \mathscr{H}}{\longrightarrow} L^{|\mathbf{k}|} \times X^{l} \times \mathcal{H}_{(X, L), \mathbf{d}}^{V, \mathbb{s}} .
$$

Moreover, for generic pair $(J, \nu) \in \mathbb{J}^{V}$, the complement of $\mathcal{M}_{\mathbf{k}, l, \mathbb{1}}^{V, \mathbf{s}}(\Sigma, X, L, \mathbf{d})$ in the irreducible part of $\overline{\mathcal{M}}_{\mathbf{k}, l, \mathbb{1}}^{V, \mathbf{s}}(\Sigma, X, L, \mathbf{d})$ has codimension at least two.

Proof. Since we only consider the case without boundary intersection point, it is parallel to the proof of Theorem 7.4 of [IP1].

In fact, the following lemma is useful for the proof of the theorem above and the discussion below. Since it is a version parallel to the lemma 7.6 of [P1], we will not repeat the proof. We use $\mathcal{S}=\cup \Lambda_{K}$ (with a marked layer structure but no specified complex structure) to label the strata of the space of $V$-stable maps, and denote these strata by $\overline{\mathcal{M}}_{\mathbf{k}, l, \mathbb{1}}^{V, \mathbb{s}}(\mathcal{S})$

Lemma 4.4 Each irreducible stratum $\overline{\mathcal{M}}_{\mathbf{k}, l, \mathbb{1}}^{V, \mathbb{S}}(\mathcal{S})$ is an orbifold whose dimension is

$$
2 r+\mathcal{L}_{0}^{b}+2 \sum_{K} \mathcal{L}_{K}^{i}
$$

less than that in (4.7), where $r$ is the total number of nontrivial layers without boundary and $\mathcal{L}_{K}^{b}$ (resp. $\mathcal{L}_{K}^{i}$ ) is the number of boundary (resp. interior) double points, whether or not intersecting other layers, in layer $\Lambda_{K}, K \geq 0$.

\subsection{Gluing construction}

We can refer to Chapter 7 of [FOOO] for the construction of Kuranishi structures and especially for gluing construction of local charts of interior and codimension part of moduli space of open stable maps. Since we essentially only consider semi-positive $(X, L)$ and $V$, we do not need to construct the Kuranishi structure, the situation is much simpler, we just give a rough description.

Note from Theorem 4.1 and Lemma 4.4 that, for our special case $L \cap V=$ $\emptyset$, the $V$-stable maps with some components sinking in $V$ belong to strata of codimension at least 4 . The codimension 1 stratum consists of $V$-regular maps with only one boundary node, denoted by $\mathcal{M}_{\mathbf{k}, l, \mathbb{1}}^{V, \mathbb{s}}(\widehat{\Sigma}, X, L, \mathbf{d})^{1}$, where $\widehat{\Sigma}=\Sigma \cup$ 
$D^{2} / z_{0}^{\prime} \sim z_{0}^{\prime \prime}, z_{0}^{\prime}$ is the extra marked point on the $b^{\text {th }}$ boundary component $(\partial \Sigma)_{b}$, $z_{0}^{\prime \prime}$ is the extra marked point on $\partial D^{2}$. The top stratum of it is the (irreducible) moduli space of $V$-regular maps with smooth domain surface. Higher codimension strata consist of both $V$-regular maps with more than 1 disc or sphere bubbles on domain and $V$-stable maps with some components sinking in $V$.

We just consider the gluing process for boundary 1-nodal strata. That is, we will glue an open $V$-regular map with smooth domain with a $(J, \nu)$-holomorphic disc to obtain an open $V$-regular map with a smooth domain.

For simplicity, we only consider the special case that $\mathbf{k}=\mathbf{0}, l=0$, i.e. without marked points. Denote the moduli space by $\mathcal{M}_{\mathbb{1}}^{V, \mathbb{s}}(\Sigma, X, L, \mathbf{d})$. Let $S$ be a stratum data of the codimension 1 stratum, which records the graph structure of the domain. We admit different complex structures on $\widehat{\Sigma}$, denote the space of complex structures on $\widehat{\Sigma}$ by $M_{\widehat{\Sigma}}$. As for the closed case, one can define an orbifold half-line bundle

$$
L_{\widehat{\Sigma}} \rightarrow M_{\widehat{\Sigma}}
$$

which is the normal bundle of $M_{\widehat{\Sigma}}$ in the stable moduli space of open Riemann surfaces, denoted by $\bar{M}_{\Sigma}$. The corresponding codimension 1 stratum is denoted by $\mathcal{M}_{\mathbb{1}}^{V, \Phi}\left(\widehat{\Sigma}_{S}, X, L, \mathbf{d}\right)^{1}$, which is the space of pairs $(u, j)$, where $\widehat{\Sigma}_{S}$ denotes the 1-nodal surfaces admitting various complex structures.

Then the forgetting map

$$
\begin{gathered}
\mathcal{F}: \mathcal{M}_{\mathbb{1}}^{V, \mathbb{\Phi}}\left(\widehat{\Sigma}_{S}, X, L, \mathbf{d}\right)^{1} \rightarrow M_{\widehat{\Sigma}}, \\
\mathcal{F}(u, j)=j
\end{gathered}
$$

induces an orbifold half-line bundle

$$
\mathcal{F}^{*} L_{\widehat{\Sigma}} \rightarrow \mathcal{M}_{\mathbb{I}}^{V, \mathbb{s}}\left(\widehat{\Sigma}_{S}, X, L, \mathbf{d}\right)^{1} .
$$

Given a point $\mu=(u, j, \rho) \in \mathcal{F}^{*} L_{\widehat{\Sigma}}$, we want to construct a holomorphic map $G l(\mu) \in \mathcal{M}_{\mathbb{I}}^{V, \mathbb{s}}(\Sigma, X, L, \mathbf{d})$. We first do the process of pre-gluing that gives an approximation map $\operatorname{pgl}(\mu)$.

(1) Pre-gluing. Firstly, the gluing domain surface $\Sigma_{z_{0}, \rho}$ can be obtained as follows. Let $2 T=\log (1+|\rho|)$. We use the holomorphic strip-like coordinates on $\Sigma$ and $D^{2}$ near $z_{0}^{\prime}$ and $z_{0}^{\prime \prime}$, that is

$$
\begin{aligned}
& \Sigma \backslash\left\{z_{0}^{\prime}\right\}=\Sigma_{o} \cup\{[-2 T,+\infty) \times[0,1]\}, \\
& D^{2} \backslash\left\{z_{0}^{\prime \prime}\right\}=D_{o}^{2} \cup\{(-\infty, 2 T] \times[0,1]\},
\end{aligned}
$$

where $\Sigma_{o}$ and $D_{o}^{2}$ are the remaining parts of $\Sigma$ and $D^{2}$ taking away a small neighborhood of boundary nodes $z_{0}^{\prime}$ and $z_{0}^{\prime \prime}$, respectively.

Then we cut off the part $[0, \infty) \times[0,1]$ of $\Sigma$ and $(-\infty, 0] \times[0,1]$ of $D^{2}$ with strip-like coordinates and glue the remainders by identifying $\{0\} \times[0,1]$. The derived surface is also denoted by $\Sigma_{z_{0}, T}$. 
For $u=\left(u_{1}, u_{2}\right) \in \mathcal{M}_{\mathbb{1}}^{V, \mathbb{s}}\left(\widehat{\Sigma}_{S}, X, L, \mathbf{d}\right)^{1}$, the pre-gluing map $\operatorname{pgl}\left(u, j_{o}, T\right)$ should be a map on surface $\Sigma_{z_{0}, T}$ with the same tangents with $V$. We simply denote this map by $\psi=\operatorname{pgl}\left(u, j_{o}, T\right): \Sigma_{z_{0}, T} \rightarrow(X, L)$, and define it partially by

$$
\psi(x)= \begin{cases}u_{1}(x) & x \in \Sigma_{o} \\ p=u_{1}\left(z_{0}^{\prime}\right)=u_{2}\left(z_{0}^{\prime \prime}\right) & x \in[-T, T] \times[0,1], \\ u_{2}(x) & x \in D_{o}^{2} .\end{cases}
$$

On the rest part the map can be defined by using a fixed smooth cutoff function. We do not explicitly write the form of the map on this part and just claim that it is a minor modification of the construction for the closed domain case, and parallel estimates can show that the $L^{p}$-norm of the differential $\bar{\partial}_{j_{o T}, J, \nu} \psi$ can be bounded from above by $C T^{1 / 2 p}$.

(2) Right inverses. With the pre-gluing map $\psi$, one can consider its linearized map $D_{\psi, j_{o \rho}}$ and then construct the right inverse $R_{\psi, j_{o T}}$ to $D_{\psi, j_{o T}}$. The construction is standard, we will refer to [FOOO] and not repeat the argument.

(3) Gluing maps. With the pre-gluing map $\psi$ and the right inverse $R_{\psi, j_{o T}}$, we can construct a holomorphic curve by perturbing the pre-gluing map. The construction is still standard. Roughly, when the $L^{p}$-norm of the differential $\bar{\partial}_{j_{o T}, J, \nu} \psi$ is sufficiently small, there exists a unique map $g\left(u, j_{o}, T\right)$ in $L^{p}\left(\Lambda_{j_{o T}}^{0,1} \psi^{*} T M\right)$ such that the map $\exp _{\psi} R_{\psi, j_{o T}} g\left(u, j_{o}, T\right)$ is holomorphic. Thus the gluing map is defined to be

$$
\begin{gathered}
G l:\left.\mathcal{F}^{*} L_{\widehat{\Sigma}}\right|_{U} \longrightarrow \mathcal{M}_{\mathbb{1}}^{V, \Phi}(\Sigma, X, L, \mathbf{d}) \\
G l(u, j, T)=\operatorname{pgl}(u, j, T)+g(u, j, T) .
\end{gathered}
$$

where $U \subset \mathcal{M}_{\mathbb{1}}^{V, \mathbb{\Phi}}\left(\widehat{\Sigma}_{S}, X, L, \mathbf{d}\right)^{1}$ is any proper open subset.

In general, we can glue maps with marked points. We consider the case that there is only one node $z_{0}$ which is different from any marked point, one domain surface is $\Sigma$ with an extra point $z_{0}^{\prime} \in(\partial \Sigma)_{b}$, the other is a disc $D^{2}$ with an extra point $z_{0}^{\prime \prime} \in \partial D^{2}$. Then by the same method, the gluing map can be constructed as

$$
\begin{aligned}
G l: \mathcal{M}_{\mathbf{k}_{1}+e_{b}, l_{1}, \mathbb{1}_{1}}^{V, \mathbb{s}^{1}}\left(\Sigma, X, L, \mathbf{d}_{1}\right)_{e v b_{z_{0}^{\prime}}} & \times_{e v b_{z_{0}^{\prime \prime}}} \mathcal{M}_{k_{2}+1, l_{2}, \mathbb{1}_{2}}^{V, \mathbb{s}^{2}}\left(D^{2}, X, L, d_{2}\right) \times(0,+\infty) \\
\longrightarrow & \mathcal{M}_{\mathbf{k}, l, \mathbb{1}}^{V, \mathbb{1}}(\Sigma, X, L, \mathbf{d}),
\end{aligned}
$$

where $|\mathbf{k}|=\left|\mathbf{k}_{\mathbf{1}}\right|+k_{2}, l=l_{1}+l_{2}, \mathbb{1}=\mathbb{1}_{1}+\mathbb{1}_{2}, \mathbf{d}=\mathbf{d}_{1}+d_{2}, e_{b}=(0, \cdots, 1, \cdots, 0)$ is a vector with only the $b^{\text {th }}$ component is 1 , others are zero. This induces local coordinate charts for the moduli space. 


\section{Orietation}

\subsection{Orienting the determinant line bundle over moduli space}

Let $\mathrm{V} \rightarrow B$ be a vector bundle. Denote by $w_{i}(\mathrm{~V})$ the $i^{\text {th }}$ Stiefel-Whitney class of $\mathrm{V}$. Define two characteristic classes $p^{ \pm}(\mathrm{V}) \in H^{2}(B, \mathbb{Z} / 2 \mathbb{Z})$ by

$$
p^{+}(\mathrm{V})=w_{2}(\mathrm{~V}), \quad p^{-}(\mathrm{V})=w_{2}(\mathrm{~V})+w_{1}(\mathrm{~V})^{2} .
$$

From [KT] we know that $p^{ \pm}(\mathrm{V})$ is the obstruction to the existence of a $\operatorname{Pin}^{ \pm}$ structure on $\mathrm{V}$.

Definition 5.1 Given a symplectic Lagrangian pair $(X, L)$, we say $L$ is relatively $\operatorname{Pin}^{ \pm}$if

$$
p^{ \pm}(T L) \in \operatorname{Im}\left(i^{*}: H^{2}(X, \mathbb{Z} / 2 \mathbb{Z}) \rightarrow H^{2}(L, \mathbb{Z} / 2 \mathbb{Z})\right) .
$$

and is $\operatorname{Pin}^{ \pm}$if $p^{ \pm}(T L)=0$. If $L$ is Pin $^{ \pm}$, we define a Pin ${ }^{ \pm}$structure for $L$ to be a Pin ${ }^{ \pm}$structure for $T L$. If $L$ is relatively $\operatorname{Pin}^{ \pm}$, we define a relative $\operatorname{Pin}^{ \pm}$ structure for $(X, L)$ consists of the choices of

1. a triangulation for the pair $(X, L)$,

2. an oriented vector bundle $\mathbb{V}$ over the three skeleton of $X$ such that $w_{2}(\mathbb{V})=$ $p^{ \pm}(T L)$,

3. a Pin ${ }^{ \pm}$structure on $\left.\left.T L\right|_{L^{(3)}} \oplus \mathbb{V}\right|_{L^{(3)}}$.

Let us first introduce some notations. Recall that

$$
\mathcal{M}_{\mathbf{k}, l, \mathbb{1}}^{V, \mathbb{s}}(\Sigma, X, L, \mathbf{d})
$$

is the moduli space of $(J, \nu)$-holomorphic $V$-regular open maps $u:(\Sigma, \partial \Sigma) \rightarrow$ $(X, L)$ with $k_{a}$ marked points $z_{a 1}, \cdots, z_{a k_{a}}$ on each boundary component $(\partial \Sigma)_{a}$ and $l$ marked points $w_{1}, \cdots, w_{l}$ and additional $\mathbb{1}$ interior intersecting marked points $q_{1}, \cdots, q_{\mathbb{1}}$ on $\Sigma$ such that $u_{*}([\Sigma, \partial \Sigma])=d$ and $\left.u\right|_{(\partial \Sigma)_{a} *}\left(\left[(\partial \Sigma)_{a}\right]\right)=d_{a}$, and $\mathbb{1}>0$. The compactification $\overline{\mathcal{M}}_{\mathbf{k}, l, \mathbb{1}}^{V, \mathbf{s}}(\Sigma, X, L, \mathbf{d})$ is the space of $V$-stable open maps. We can define the evaluation maps at the $(\mathbf{k}, l)$-marked points and intersection points

$$
\begin{gathered}
e v b_{a i}: \overline{\mathcal{M}}_{\mathbf{k}, l, \mathbb{1}}^{V, \mathbb{s}}(\Sigma, X, L, \mathbf{d}) \rightarrow L, \quad i=1, \cdots, k_{a}, a=1, \cdots, m, \\
e v i_{j}: \overline{\mathcal{M}}_{\mathbf{k}, l, \mathbb{1}}^{V, \mathbb{s}}(\Sigma, X, L, \mathbf{d}) \rightarrow X, \quad j=1, \cdots, l \\
e v i_{\jmath}^{I}: \overline{\mathcal{M}}_{\mathbf{k}, l, \mathbb{1}}^{V, \mathbb{1}}(\Sigma, X, L, \mathbf{d}) \rightarrow V, \quad \jmath=1, \cdots, \mathbb{1} .
\end{gathered}
$$

In fact, the moduli space above can be considered as the zero locus of a Fredholm section of a Banach bundle. We denote by $B^{1, p}(\Sigma, X, L, \mathbf{d})$ the Banach manifold of $W^{1, p}$ maps $u:(\Sigma, \partial \Sigma) \rightarrow(X, L)$ such that $u_{*}([\Sigma, \partial \Sigma])=d$ and $\left.u\right|_{(\partial \Sigma)_{a *}}\left(\left[(\partial \Sigma)_{a}\right]\right)=d_{a}$. And define

$$
B_{\mathbf{k}, l+\mathbb{1}}^{1, p}(\Sigma, X, L, \mathbf{d}):=B^{1, p}(\Sigma, X, L, \mathbf{d}) \times \prod_{a}(\partial \Sigma)_{a}^{k_{a}} \times \Sigma^{l+\mathbb{1}} \backslash \triangle,
$$


where $\triangle$ denotes the subset of the product in which two marked points coincide. Elements of $B_{\mathbf{k}, l+\mathbb{1}}^{1, p}(\Sigma, X, L, \mathbf{d})$ are denoted by $\mathbf{u}=(u, \vec{z}, \vec{w}, \vec{q})$, where $\vec{z}=\left(z_{a i}\right)$, $\vec{w}=\left(w_{j}\right), \vec{q}=\left(q_{\jmath}\right)$.

Then the Banach space bundle $\mathcal{E} \rightarrow B_{\mathbf{k}, l+\mathbb{1}}^{1, p}(\Sigma, X, L, \mathbf{d})$ is defined fiberwise with

$$
\mathcal{E}_{\mathbf{u}}:=L^{p}\left(\Sigma, \Omega^{0,1}\left(u^{*} T X\right)\right) .
$$

We define the section of this bundle as

$$
\begin{gathered}
\bar{\partial}_{(J, \nu)}: B_{\mathbf{k}, l+\mathbb{1}}^{1, p}(\Sigma, X, L, \mathbf{d}) \rightarrow \mathcal{E} \\
\bar{\partial}_{(J, \nu)} u=d u \circ j_{\Sigma}+J \circ d u-\nu(\cdot, u(\cdot), \mathbf{u}),
\end{gathered}
$$

which is the $\nu$-perturbed Cauchy-Riemann operator. We require the inhomogeneous term

$$
\nu \in \Gamma\left(\Sigma \times X \times B_{\mathbf{k}, l+\mathbb{1}}^{1, p}(\Sigma, X, L, \mathbf{d}), \operatorname{Hom}\left(\pi_{1}^{*} T \Sigma, \pi_{2}^{*} T X\right)\right),
$$

such that

(1) $\nu$ is $\left(j_{\Sigma}, J\right)$-anti-linear, i.e. $\nu \circ j_{\Sigma}=-J \circ \nu$;

(2) $\left.\nu\right|_{\partial \Sigma \times X \times B_{\mathbf{k}, l+1}^{1, p}(\Sigma, X, L, \mathbf{d})}$ carries the sub-bundle $\pi_{1}^{\prime *} T \partial \Sigma \subset \pi_{1}^{\prime *} T \Sigma$ to the subbundle $\pi_{2}^{\prime *}(J T L) \subset \pi_{2}^{\prime *} T X$, where $\pi_{i}, i=1,2$, is the projection from $\Sigma \times X \times$ $B_{\mathbf{k}, l+\mathbb{1}}^{1, p}(\Sigma, X, L, \mathbf{d})$ to the $i^{\text {th }}$ factor.

The vertical component of the linearization of this section is denoted by

$$
D:=D \bar{\partial}_{(J, \nu)}: T B_{\mathbf{k}, l+\mathbb{1}}^{1, p}(\Sigma, X, L, \mathbf{d}) \rightarrow \mathcal{E} .
$$

Definition $5.2 A W^{1, p}$-map $u \in B_{\mathbf{k}, l+\mathbb{1}}^{1, p}(\Sigma, X, L, \mathbf{d})$ is called $V$-regular if no component of its domain is mapped entirely into $V$ and if neither any of the $|k|+l$ marked points nor any double points are mapped into $V$.

Thus the parameterized $V$-regular moduli space can be regarded as the zero locus of the section $\bar{\partial}_{(J, \nu)}$ which is denoted by

$$
\widetilde{\mathcal{M}}_{\mathbf{k}, l, \mathbb{1}}^{V, \mathbb{s}}(\Sigma, X, L, \mathbf{d}):=\bar{\partial}_{J, \nu}^{-1}(0) \cap B_{\mathbf{k}, l+\mathbb{1}}^{1, p, V}(\Sigma, X, L, \mathbf{d}) .
$$

Alternatively, similar to [So], we can also define our moduli space $\mathcal{M}_{\mathbf{k}, l, \mathbb{1}}^{V, \mathbf{s}}(\Sigma, X, L, \mathbf{d})$ as an appropriate section (or say slice) of the reparameterization group action, we refer the reader to the section 4 in $[\mathrm{So}$ for details of construction.

We simply denote $B^{V}:=B_{\mathbf{k}, l+\mathbb{1}}^{1, p, V}(\Sigma, X, L, \mathbf{d})$. The evaluation maps can also be similarly defined on this larger space

$$
\begin{gathered}
e v b_{a i}: B^{V} \rightarrow L, \quad i=1, \cdots, k_{a}, a=1, \cdots, m, \\
e v i_{j}: B^{V} \rightarrow X, \quad j=1, \cdots, l, \\
e v i_{\jmath}^{I}: B^{V} \rightarrow V, \quad \jmath=1, \cdots, \mathbb{1}, \quad \text { if } \mathbb{1} \geq 1 .
\end{gathered}
$$


such that

$$
e v b_{a i}(\mathbf{u})=u\left(z_{a i}\right), \quad e v i_{j}(\mathbf{u})=u\left(w_{j}\right), \quad e v i_{\jmath}^{I}(\mathbf{u})=u\left(q_{\jmath}\right) .
$$

The total evaluation map is denoted by

$$
\begin{gathered}
\mathbf{e v}:=\prod_{a, i} e v b_{a i} \times \prod_{j} e v i_{j} \times \prod_{\mathbb{1} \geq 1, \jmath} e v i_{\jmath}^{I} \\
\mathbf{e v}: B_{\mathbf{k}, l+\mathbb{1}}^{1, p, V}(\Sigma, X, L, \mathbf{d}) \rightarrow L^{|\mathbf{k}|} \times X^{l} \times V^{\mathbb{1}} .
\end{gathered}
$$

Then let

$$
\mathcal{L}:=\operatorname{det}(D) \rightarrow B_{\mathbf{k}, l+\mathbb{1}}^{1, p, V}(\Sigma, X, L, \mathbf{d})
$$

be the determinant line bundle of the family of Fredholm operators $D$ restricted to $B^{V}$. And let

$$
\mathcal{L}^{\prime}=\mathcal{L} \otimes \bigotimes_{a, i} e v b_{a i}^{*} \operatorname{det}(T L)^{*}
$$

The following two propositions are the fundamental results of orienting the moduli space. We distinguish the following two situations

(1) $L$ is orientable and provided with an orientation. In this situation no restriction is required;

(2) $L$ is nonorientable, then we suppose the number of marked points on each boundary component satisfies

$$
k_{a} \cong w_{1}\left(d_{a}\right)+1, \quad(\bmod 2) ;
$$

Denote by $M^{(i)}$ the $i$-skeleton of a manifold $M$. Then we define a subspace

$$
\mathfrak{B}:=\left\{(u, \vec{z}, \vec{w}, \vec{q}) \in B_{\mathbf{k}, l+\mathbb{1}}^{1, p, V}(\Sigma, X, L, \mathbf{d}) \mid u:(\Sigma, \partial \Sigma) \rightarrow\left(X^{(3)}, L^{(3)}\right)\right\} .
$$

We first prove a lemma

Lemma 5.1 Let $(X, L)$ be a symplectic Lagrangian pair and $L$ be relatively $\operatorname{Pin}^{ \pm}$. The combination of the relative Pin ${ }^{ \pm}$structure of $(X, L)$ and the orientations of $L$ if it is orientable determines a canonical orientation of $\left.\mathcal{L}^{\prime}\right|_{\mathfrak{B}}$.

Proof. We just need canonically orient each individual line $\mathcal{L}_{\mathbf{u}}^{\prime}$ for each $\mathbf{u} \in \mathfrak{B}$ in a way that varies continuously in families. Recall that the relative $\operatorname{Pin}^{ \pm}$structure of $(X, L)$ gives a vector bundle $\mathbb{V} \rightarrow X^{(3)}$ and a $\operatorname{Pin}^{ \pm}$structure on $\left.\left.\mathbb{V}\right|_{L^{(3)}} \oplus T L\right|_{L^{(3)}}$. Denote simply by

$$
\mathbb{V}_{\mathbb{R}}:=\left.\mathbb{V}\right|_{L^{(3)}}, \quad \mathbb{V}_{\mathbb{C}}:=\mathbb{V} \otimes \mathbb{C} .
$$

Choosing an arbitrary Cauchy-Riemann operator $D_{0}$ on $u^{*} \mathbb{V}_{\mathbb{C}}$, we consider the operator $D_{\mathbf{u}} \oplus D_{0}$,

$$
D_{\mathbf{u}} \oplus D_{0}: T B_{\mathbf{u}} \oplus W^{1, p}\left(u^{*} \mathbb{V}_{\mathbb{C}}, u^{*} \mathbb{V}_{\mathbb{R}}\right) \longrightarrow \mathcal{E} \oplus L^{p}\left(\Omega^{0,1}\left(u^{*} \mathbb{V}_{\mathbb{C}}\right)\right)
$$


That is

$$
W_{\mathbb{S}}^{1, p}\left(u^{*}\left(T X \oplus \mathbb{V}_{\mathbb{C}}\right),\left.u\right|_{\partial \Sigma} ^{*}\left(T L \oplus \mathbb{V}_{\mathbb{R}}\right)\right) \oplus \mathbb{R}^{|\mathbf{k}|} \oplus \mathbb{C}^{l+\mathbb{1}} \rightarrow L^{p}\left(\Omega^{0,1}\left(u^{*}\left(T X \oplus \mathbb{V}_{\mathbb{C}}\right)\right)\right)
$$

We remark that the choice of $D_{0}$ is irrelevant since the space of real linear CauchyRiemann operators on $u^{*} \mathbb{V}_{\mathbb{C}}$ is contractible.

Thus, we have a short exact sequence of Fredholm operators

$$
0 \rightarrow D_{\mathbf{u}} \rightarrow D_{\mathbf{u}} \oplus D_{0} \rightarrow D_{0} \rightarrow 0
$$

By Lemma A.1 there exists an isomorphism

$$
\operatorname{det}\left(D_{\mathbf{u}}\right) \simeq \operatorname{det}\left(D_{\mathbf{u}} \oplus D_{0}\right) \otimes \operatorname{det}\left(D_{0}\right)^{*} .
$$

Tensor by $\bigotimes_{a, i}\left(e v b_{a i}^{*} \operatorname{det}(T L)^{*}\right)_{\mathbf{u}}$ on both sides,

$$
\begin{aligned}
\mathcal{L}_{\mathbf{u}}^{\prime} & =\operatorname{det}\left(D_{\mathbf{u}}\right) \otimes \bigotimes_{a, i}\left(e v b_{a i}^{*} \operatorname{det}(T L)^{*}\right)_{\mathbf{u}} \\
& \simeq \operatorname{det}\left(D_{\mathbf{u}} \oplus D_{0}\right) \otimes \operatorname{det}\left(D_{0}\right)^{*} \otimes \bigotimes_{a, i}\left(e v b_{a i}^{*} \operatorname{det}(T L)^{*}\right)_{\mathbf{u}}
\end{aligned}
$$

Actually, we only need to orient

$$
\mathcal{L}_{\mathbf{u}}^{\prime} \otimes \operatorname{det}\left(D_{0}\right) \simeq \operatorname{det}\left(D_{\mathbf{u}} \oplus D_{0}\right) \otimes \bigotimes_{a, i}\left(e v b_{a i}^{*} \operatorname{det}(T L)^{*}\right)_{\mathbf{u}},
$$

since the Cauchy-Riemann Pin boundary value problem

$$
\underline{D}_{0}=\left(\Sigma, u^{*} \mathbb{V}_{\mathbb{C}}, u^{*} \mathbb{V}_{\mathbb{R}}, \mathfrak{P}_{0}, D_{0}\right)
$$

determines a canonical orientation on $\operatorname{det}\left(D_{0}\right)$. Indeed, note that any real vector bundle over a Riemann surface $\Sigma$ with nonempty boundary admits a Pin structure because the second cohomology $H^{2}(\Sigma)$ is trivial. So we can choose a Pin structure $\widetilde{\mathfrak{P}}_{0}$ on $u^{*} \mathbb{V} \rightarrow \Sigma$ and define $\mathfrak{P}_{0}$ to be its restriction to $u^{*} \mathbb{V}_{\mathbb{R}} \rightarrow \partial \Sigma$. By the Lemma 2.11 in $\left[\mathrm{So}\right.$, the canonical orientation on $\operatorname{det}\left(D_{0}\right)$ does not depend on the choice of $\widetilde{\mathfrak{P}}_{0}$.

Recall that the relative Pin structure on $(X, L)$ gives a Pin structure on $\left.\left.T L\right|_{L^{(3)}} \oplus \mathbb{V}\right|_{L^{(3)}}$, and so gives a Pin structure on $\left.u\right|_{\partial \Sigma} ^{*}\left(T L \oplus \mathbb{V}_{\mathbb{R}}\right)$. The notation is simplified since $u \in \mathfrak{B}$.

If $L$ is orientable and given an orientation, we have induced orientations on $\left.u\right|_{\partial \Sigma} ^{*}\left(T L \oplus \mathbb{V}_{\mathbb{R}}\right)$. Thus we consider the restricted Pin boundary value problem $\underline{D_{\mathbf{u}} \oplus D_{0}}$, the Proposition A.1 and the Remark after it ensures there exists a canonical orientation on $\operatorname{det}\left(D_{\mathbf{u}} \oplus D_{0}\right)$. Then the orientation of $L$ gives the orientation of $\operatorname{det}(T L)$. Therefore, we provide a canonical orientation on the right-hand side of (5.9).

If $L$ is non-orientable, on each boundary component $(\partial \Sigma)_{a}$ such that $k_{a} \neq 0$, choose arbitrarily an orientation on $\left(e v b_{a 1}^{*} T L\right)_{\mathbf{u}}$. Still note that each boundary component $(\partial \Sigma)_{a}$ has an orientation induced from the natural orientation on 
$\Sigma$. For each $i \in\left[2, k_{a}\right]$ if $k_{a} \neq 0$, we can obtain an orientation on $\left(e v b_{a i}^{*} T L\right)_{\mathbf{u}}$ by trivializing $\left.u\right|_{\partial \Sigma} ^{*} T L$ along the oriented line segment in $(\partial \Sigma)_{a}$ from $z_{a 1}$ to $z_{a i}$. If for some $a,\left.u\right|_{(\partial \Sigma)_{a}} ^{*} T L$ is orientable, and if $k_{a} \neq 0$, then the orientation on $\left(e v b_{a 1}^{*} T L\right)_{\mathbf{u}}$ induces an orientation on $\left.u\right|_{(\partial \Sigma)_{a}} ^{*} T L$; otherwise, if $k_{a}=0$, then we arbitrarily choose an orientation on $\left.u\right|_{(\partial \Sigma)_{a}} ^{*} T L$. Thus for such $a,\left.u\right|_{(\partial \Sigma)_{a}} ^{*}(T L \oplus$ $\mathbb{V}_{\mathbb{R}}$ ) also has an orientation by choosing an orientation on $\mathbb{V}_{\mathbb{R}}$. Similar to the above argument $\left.u\right|_{\partial \Sigma} ^{*}\left(T L \oplus \mathbb{V}_{\mathbb{R}}\right)$ admits a Pin structure given by the relative Pin structure on $(X, L)$. Then the Proposition A.1 and the Remark after it still apply to $\underline{D_{\mathbf{u}} \oplus D_{0}}$, and $\operatorname{det}\left(D_{\mathbf{u}} \oplus D_{0}\right)$ has a canonical orientation. Hence we also provide a canonical orientation on the right-hand side of (5.9). Note that under the additional assumption (5.6), the choice of orientation on $\left(e v b_{a 1}^{*} T L\right)_{\mathbf{u}}$ is not important. Because changing the orientation on $\left(e v b_{a 1}^{*} T L\right)_{\mathbf{u}}$ will change all the orientations on $\left.u\right|_{(\partial \Sigma)_{a}} ^{*}\left(T L \oplus \mathbb{V}_{\mathbb{R}}\right)$ and on $\left(e v b_{a i}^{*} T L\right)_{\mathbf{u}}, i \in\left[2, k_{a}\right]$ it induces, by Proposition $A .1$ and Remark after it, the orientation on $\operatorname{det}\left(D_{\mathbf{u}} \oplus D_{0}\right)$ will also change. Then the assumption (5.6) ensures that the number of orientation changes is even. So the orientation on the right-hand side of (5.9) is invariant.

Note that the construction above varies continuously in a one-parameter family, we thus canonically oriented $\left.\mathcal{L}^{\prime}\right|_{\mathfrak{B}}$.

Proposition 5.1 Under the assumptions in Lemma 5.1, the combination of the orientations of $L$ if it is orientable and the choice of relative Pin ${ }^{ \pm}$structure $\mathfrak{P}$ on $L$ provides a canonical orientation on $\mathcal{L}^{\prime}$, that is to say, there exists a canonical isomorphism of line bundles

$$
\mathcal{L} \simeq \bigotimes_{a, i} e v b_{a i}^{*} \operatorname{det}(T L)
$$

Proof. It suffices to provide a canonical orientation for the fiber $\mathcal{L}_{\mathbf{u}}^{\prime}$ over each $\mathbf{u} \in B^{V}=B_{\mathbf{k}, l+\mathbb{1}}^{1, p, V}(\Sigma, X, L, \mathbf{d})$ such that it varies continuously with $\mathbf{u}$.

By definition the relative $\operatorname{Pin}^{ \pm}$structure gives a triangulation of the pair $(X, L)$. The map $u:(\Sigma, \partial \Sigma) \rightarrow(X, L)$ is homotopic to a map $\hat{u}:(\Sigma, \partial \Sigma) \rightarrow$ $\left(X^{(2)}, L^{(2)}\right)$ by using simplicial approximation. The homotopy map is denoted by

$$
\Phi:[0,1] \times(\Sigma, \partial \Sigma) \rightarrow(X, L) .
$$

We will show that the choice of map $\Phi$ is unique up to homotopy. Indeed, let $\Phi^{\prime}$ be another such map. We can get a new map by concatenating $\Phi$ and $\Phi^{\prime}$

$$
\Phi \# \Phi^{\prime}:[-1,1] \times(\Sigma, \partial \Sigma) \rightarrow(X, L) .
$$

We can use simplicial approximation again to homotope $\Phi \# \Phi^{\prime}$ to a map into the three skeleton $\left(X^{(3)}, L^{(3)}\right)$. By retaking suitable parameters, we get a homotopy map from $\Phi$ to $\Phi^{\prime}$, denote it by

$$
\Psi:[0,1]^{2} \times(\Sigma, \partial \Sigma) \rightarrow(X, L),
$$

satisfying

$$
\Psi(0, t)=\Phi(t), \quad \Psi(1, t)=\Phi^{\prime}(t), \quad \Psi(s, 0)=u
$$


That is to say $\Phi$ is unique up to homotopy.

On the other hand, the two maps $\Phi$ and $\Psi$ can be considered as maps from $[0,1]$ and $[0,1]^{2}$ to $B^{V}$, respectively. Note that $\hat{u} \in \mathfrak{B}$, by the Lemma 5.1, the relative $\operatorname{Pin}^{ \pm}$structure of $(X, L)$ determines a canonical orientation of $\left.\mathcal{L}^{\prime}\right|_{\mathfrak{B}}$. Then the orientation on $\left.\mathcal{L}^{\prime}\right|_{\hat{\mathbf{u}}}$ induces an orientation of $\left.\mathcal{L}^{\prime}\right|_{\mathbf{u}}$ by trivializing $\Phi^{*} \mathcal{L}^{\prime}$. Such orientation is the same as the one induced by any other homotopy $\Phi^{\prime}$ since we can trivialize $\Psi^{*} \mathcal{L}^{\prime}$. It is easy to see such induced orientation on $\left.\mathcal{L}^{\prime}\right|_{\mathbf{u}}$ varies continuously with $\mathbf{u}$. Therefore, $\mathcal{L}^{\prime}$ admits a canonical orientation.

The isomorphism (5.10) doesn't involve the effect of the ordering of the marked points on boundary components. In fact, $B_{\mathbf{k}, l+\mathbb{1}}^{1, p, V}(\Sigma, X, L, \mathbf{d})$ consists many connected components, each one corresponds to each ordering of boundary marked points. Denote

$$
\varpi=\left(\varpi_{1}, \cdots, \varpi_{m}\right)
$$

where each $\varpi_{a}$ is a permutation of the integers $1, \cdots, k_{a}$. We define the sign of $\varpi$

$$
\operatorname{sign}(\varpi):=\sum_{a} \operatorname{sign}\left(\varpi_{a}\right) .
$$

Denote by $B_{\varpi}^{V}$ the component of $B_{\mathbf{k}, l+\mathbb{1}}^{1, p, V}(\Sigma, X, L, \mathbf{d})$ in which $\vec{z}$ are ordered in $\partial \Sigma$ by $\varpi$. Now we modify the canonical isomorphism in Proposition 5.1 by

Definition 5.3 When $\operatorname{dim} L \simeq 0(\bmod 2)$ we define the canonical isomorphism to be the isomorphism constructed in the Proposition 5.1 twisted by $(-1)^{\operatorname{sign}(\varpi)}$ over the component $B_{\mathbf{k}, l, \varpi}^{1, p}(\Sigma, L, \mathbf{d})$. Otherwise, we define the isomorphism to be exactly the isomorphism constructed in the Proposition 5.1.

Now we consider the orientation of moduli space of $V$-stable maps. We will restrict attention to the special case: $V$-stable map of two components, one of which is from the original Riemann surface $\Sigma$, and the other of which is a disc bubble, no component is mapped into $V$;

Recall that the domain is equipped with a marked layer structure (see Definition 4.3). Similar to (4.10), we can define associated operators $D_{K}^{N}$ on the layers $\Lambda_{K}, K \geq 1$.

Definition 5.4 AV -stable $W^{1, p}{ }_{-m a p}$ is a map $u \in B_{\mathbf{k}, l+\mathbb{1}}^{1, p, V}(\Sigma, X, L, \mathbf{d})$ together with

(i) a marked layer structure on its (nodal) domain $\Sigma$ with $\left.u\right|_{\Lambda_{0}}$ being $V$-regular, and

(ii) for each $K \geq 1$, an element $\xi_{K} \in \operatorname{ker} D_{K}^{N}$ defined on the layer $\Lambda_{K}$ that is a section nontrivial on every irreducible component of $\Lambda_{K}$.

In particular, denote the space of $V$-stable $W^{1, p}$-maps with only the layer $K=0$ components by $B_{\mathbf{k}, l, \mathbb{1}}^{1, p, V}(\Sigma, X, L, \mathbf{d}, 0)$, and denote the space of $V$-stable 
$W^{1, p}$-maps with only the layer $K=1$ components by $B_{\mathbf{k}, l, \mathbb{1}}^{1, p, V}(\Sigma, X, L, \mathbf{d}, 1)$.

We will give a description in more detail. Suppose that only one disc bubbles off the boundary component $(\partial \Sigma)_{b}$ along with $k^{\prime \prime}$ of the marked points on $(\partial \Sigma)_{b}$ and $l^{\prime \prime}$ of the interior marked points. The domain is a nodal surface

$$
\widehat{\Sigma}=\Sigma \cup D^{2} / z_{0}^{\prime} \sim z_{0}^{\prime \prime}
$$

with $\left|\mathbf{k}^{\prime}\right|+1$ boundary marked points and $l^{\prime}+\mathbb{1}$ interior marked points on $\Sigma$, and $k^{\prime \prime}+1$ boundary marked points and $l^{\prime \prime}$ interior marked points on $D^{2}$ such that

$$
\mathbf{k}^{\prime}=\left(k_{1}, \cdots, k^{\prime}, \cdots, k_{m}\right), \quad k^{\prime}=k_{b}-k^{\prime \prime}, \quad l=l^{\prime}+l^{\prime \prime} .
$$

We denote by $z_{0}^{\prime}$ (resp. $z_{0}^{\prime \prime}$ ) the extra marked point on $\Sigma$ (resp. $\left.D^{2}\right)$ which is different from any $z_{b i}$. Denote by

$$
\begin{aligned}
B^{V \#} & =B_{\mathbf{k}, \sigma, l, \rho, \mathbb{1}, \varrho}^{1, \varrho}\left(\widehat{\Sigma}, X, L, \mathbf{d}^{\prime}, d^{\prime \prime}, 0\right) \\
& :=B_{\mathbf{k}^{\prime}+e_{b}, l^{\prime}, \mathbb{P}^{\prime}}^{1, p, V}\left(\Sigma, X, L, \mathbf{d}^{\prime}, 0\right)_{e v b_{z_{0}^{\prime}}} \times_{e v b_{z_{0}^{\prime \prime}}} B_{k^{\prime \prime}+1, l^{\prime \prime}, \mathbb{1}^{\prime \prime}}^{1, p, V}\left(D^{2}, X, L, d^{\prime \prime}, 0\right)
\end{aligned}
$$

the space of $V$-regular $W^{1, p}$ stable maps with only one disc bubbling off and no component is mapped into $V$ as above. The disc bubble represents the class $d^{\prime \prime} \in H_{2}(X, L)$, denote

$$
\mathbf{d}^{\prime}=\left(d^{\prime}, d_{1}, \cdots, d_{b}^{\prime}, \cdots, d_{m}\right) \in H_{2}(X, L) \oplus H_{1}(L)^{\oplus m},
$$

satisfying $d^{\prime}+d^{\prime \prime}=d, d_{b}^{\prime}+\partial d^{\prime \prime}=d_{b}$, and $e_{b}=(0, \cdots, 1, \cdots, 0)$ is the vector with only the $b^{\text {th }}$ element equal to 1 , others are zeros.

In the notation of the $W^{1, p}$ space above, $\sigma \subset\left[1, k_{b}\right]$ and $\rho \subset[1, l]$ denote the subsets of boundary and interior bubbling-off marked points, respectively. And $\varrho \subset[1, \mathbb{1}]$ denote the subsets of $V$-intersecting interior bubbling-off marked points.

We write the element $\mathbf{u}=\left(\mathbf{u}^{\prime}, \mathbf{u}^{\prime \prime}\right) \in B_{\mathbf{k}, \sigma, l, \rho, \mathbb{1}, \varrho}^{1, p, V}\left(\hat{\Sigma}, X, L, \mathbf{d}^{\prime}, d^{\prime \prime}, 0\right)$, where

$$
\begin{aligned}
\mathbf{u}^{\prime} \in B^{\prime} & :=B_{\mathbf{k}^{\prime}+e_{b}, l^{\prime}, \mathbb{1}^{\prime}}^{1, p, V}\left(\Sigma, X, L, \mathbf{d}^{\prime}, 0\right), \\
\mathbf{u}^{\prime \prime} \in B^{\prime \prime} & :=B_{k^{\prime \prime}+1, l^{\prime \prime}, \mathbb{1}^{\prime \prime}}^{1, p}\left(D^{2}, X, L, d^{\prime \prime}, 0\right)
\end{aligned}
$$

such that

$$
e v b_{z_{0}^{\prime}}\left(\mathbf{u}^{\prime}\right)=e v b_{z_{0}^{\prime \prime}}\left(\mathbf{u}^{\prime \prime}\right),
$$

where $e v b_{z_{0}^{\prime}}\left(\right.$ resp. $\left.e v b_{z_{0}^{\prime \prime}}\right)$ is the evaluation map at $z_{0}^{\prime}$ (resp. $\left.z_{0}^{\prime \prime}\right)$. For each such $\mathbf{u} \in B^{V \#}$, we denote by

$$
\widehat{\Sigma}_{\mathbf{u}}:=\Sigma \cup D^{2} / z_{0}^{\prime} \sim z_{0}^{\prime \prime} .
$$

the associated domain curve. The stable map is $u:(\widehat{\Sigma}, \partial \widehat{\Sigma}) \rightarrow(X, L)$, and the node of $\widehat{\Sigma}$ is denoted by $z_{0}$.

If we denote the two natural projections by

$$
p_{1}: B^{V \#} \rightarrow B^{\prime}, \quad p_{2}: B^{V \#} \rightarrow B^{\prime \prime},
$$


then we can define the Banach bundle $\mathcal{E}^{\#} \rightarrow B^{V \#}$ by

$$
\mathcal{E}^{\#}:=p_{1}^{*} \mathcal{E}^{\prime} \oplus p_{2}^{*} \mathcal{E}^{\prime \prime}
$$

with fiber

$$
\mathcal{E}_{\mathbf{u}}^{\#}:=L^{p}\left(\widehat{\Sigma}_{\mathbf{u}}, \Omega^{0,1}\left(u^{\prime *} T X\right) \oplus \Omega^{0,1}\left(u^{\prime \prime *} T X\right)\right) .
$$

For $(J, \nu) \in \mathbb{J}^{V}$, we denote by

$$
\bar{\partial}_{(J, \nu)}^{\#}: B^{V \#} \rightarrow \mathcal{E}^{\#}
$$

the section given by the $\nu$-perturbed Cauchy-Riemann operator. The vanishing set of this section is the parameterized one-disc-bubble moduli space, denoted by

$$
\left(\bar{\partial}_{(J, \nu)}^{\#}\right)^{-1}(0)=\widetilde{\mathcal{M}}_{\mathbf{k}, \sigma, l, \rho, \mathbb{1}, \varrho}^{V, \mathbb{S}}\left(\widehat{\Sigma}, X, L, \mathbf{d}^{\prime}, d^{\prime \prime}, 0\right) .
$$

Its vertical linearization is

$$
D^{\#}:=D \bar{\partial}_{(J, \nu)}^{\#}: T B^{V \#} \rightarrow \mathcal{E}^{\#} .
$$

Similarly, we have two operators

$$
D^{\prime}: T B^{V \#} \rightarrow p_{1}^{*} \mathcal{E}^{\prime}, \quad D^{\prime \prime}: T B^{V \#} \rightarrow p_{2}^{*} \mathcal{E}^{\prime \prime} .
$$

Denote the determinant line bundle of family of Fredholm operators $D^{\#}$ by

$$
\mathcal{L}^{\#}:=\operatorname{det}\left(D^{\#}\right) \rightarrow B^{V \#} .
$$

Denote

$$
\mathcal{L}^{\#^{\prime}}=\mathcal{L}^{\#} \otimes \bigotimes_{a, i}\left(e v b_{a i}^{*} \operatorname{det}(T L)^{*}\right.
$$

We define a subspace

$$
\mathfrak{B}^{\#}:=\left\{(u, \vec{z}, \vec{w}, \vec{q}) \in B^{V \#} \mid u:(\widehat{\Sigma}, \partial \widehat{\Sigma}) \rightarrow\left(X^{(3)}, L^{(3)}\right)\right\} .
$$

We can obtain a lemma similar to the Lemma 5.1

Lemma 5.2 Let $(X, L)$ be a symplectic Lagrangian pair and $L$ be relatively $\operatorname{Pin}^{ \pm}$. The combination of the relative Pin ${ }^{ \pm}$structure of $(X, L)$ and the orientation of

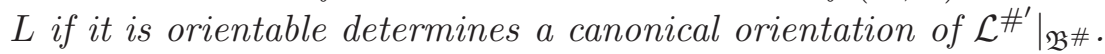

Proof. It is similar to the proof of Lemma 5.1 and the arguments in Proposition 3.3 of $[\mathrm{So}$, the modifications take place when we apply the Proposition A.1 and the Remark after it to the two restricted Pin boundary value problems

$$
\underline{D_{\mathbf{u}}^{\prime} \oplus D_{0}^{\prime}} \quad \text { and } \quad \underline{D_{\mathbf{u}}^{\prime \prime} \oplus D_{0}^{\prime \prime}} .
$$

And we have an isomorphism

$$
\operatorname{det}\left(D_{\mathbf{u}}^{\#} \oplus D_{0}^{\#}\right) \simeq \operatorname{det}\left(D_{\mathbf{u}}^{\prime} \oplus D_{0}^{\prime}\right) \otimes \operatorname{det}\left(D_{\mathbf{u}}^{\prime \prime} \oplus D_{0}^{\prime \prime}\right) \otimes e v b_{0}^{*} \operatorname{det}\left(T L \oplus V_{\mathbb{R}}\right)_{\mathbf{u}}^{*}
$$

Using the same argument of homotopy uniqueness in the proof of Proposition 5.1. we can similarly obtain the following 
Proposition 5.2 The combination of the orientations of $L$ if it is orientable and the choice of relative Pin ${ }^{ \pm}$structure $\mathfrak{P}$ on $L$ provide a canonical orientation on $\mathcal{L}^{\#^{\prime}}$, that is to say, there exists a canonical isomorphism of line bundles

$$
\mathcal{L}^{\#} \longrightarrow \bigotimes_{a, i} e v b_{a i}^{*} \operatorname{det}(T L) .
$$

In order to involve the effect of the ordering of the marked points, we also need modify the isomorphism in the Proposition 5.2. Recall from the proof above that the marked points on each $(\partial \widehat{\Sigma})_{a}$ can be canonically ordered. So we can divide the space $B_{\mathbf{k}, l}^{1, p}(\Sigma, L, \mathbf{d})$ into components $B_{\mathbf{k}, l, \varpi}^{1, p}(\Sigma, L, \mathbf{d})$. To be consistent with the isomorphism defined in Definition 5.3, we can modify the canonical isomorphism (5.16) and have the following definition.

Definition 5.5 When $\operatorname{dim} L \simeq 0(\bmod 2)$ we define the canonical isomorphism to be the isomorphism constructed in the Proposition 5.2 twisted by $(-1)^{\operatorname{sign}(\varpi)}$ over the component $B_{\mathbf{k}, l, \varpi}^{1, p}(\Sigma, L, \mathbf{d})$. Otherwise, we define the isomorphism to be exactly the isomorphism constructed in the Proposition 5.2.

\subsection{Compatiblility with gluing}

We study the compatibility of the orientation constructed above with the gluing process described in subsection 4.4. The argument is a minor modification of the one in section 8.3 of [FOOO]. For $u=\left(u_{1}, u_{2}\right)$, we denote the evaluation maps at the boundary marked points for two components and node by

$$
\begin{gathered}
e v b_{a_{1} i_{1}}^{1}: \widetilde{\mathcal{M}}_{\mathbf{k}, \sigma, l, \rho, \mathbb{1}, \varrho}^{V, \mathbb{S}}\left(\widehat{\Sigma}, X, L, \mathbf{d}^{\prime}, d^{\prime \prime}, 0\right) \rightarrow L, \\
e v b_{a_{2} i_{2}}^{2}: \widetilde{\mathcal{M}}_{\mathbf{k}, \sigma, l, \rho, \mathbb{1}, \varrho}^{V, \mathbb{\Sigma}}\left(\widehat{\Sigma}, X, L, \mathbf{d}^{\prime}, d^{\prime \prime}, 0\right) \rightarrow L, \\
e v b_{0}: \widetilde{\mathcal{M}}_{\mathbf{k}, \sigma, l, \rho, \mathbb{1}, \varrho}^{V, \mathbb{\Sigma}}\left(\widehat{\Sigma}, X, L, \mathbf{d}^{\prime}, d^{\prime \prime}, 0\right) \rightarrow L .
\end{gathered}
$$

For $u_{1}$ and $u_{2}$, we denote the evaluation maps at the extra boundary marked points $z_{0}^{\prime}$ and $z_{0}^{\prime \prime}$ by

$$
\begin{gathered}
e v b_{z_{0}^{\prime}}: \widetilde{\mathcal{M}}_{\mathbf{k}_{1}, l_{1}, \mathbb{I}_{1}}^{V, \mathbb{s}^{1}}\left(\Sigma, X, L, \mathbf{d}^{\prime}, 0\right) \rightarrow L, \\
e v b_{z_{0}^{\prime \prime}}: \widetilde{\mathcal{M}}_{k_{2}, l_{2}, \mathbb{I}_{2}}^{V, \mathbb{1}^{2}}\left(D^{2}, X, L, d^{\prime \prime}, 0\right) \rightarrow L .
\end{gathered}
$$

Recall we have defined operators $D^{\#}, D^{\prime}$ and $D^{\prime \prime}$ in (5.13) and (5.14). Let

$$
\begin{gathered}
\mathcal{T}=\left.\mathcal{L}^{\#^{\prime}}\right|_{\widetilde{\mathcal{M}}_{\mathbf{k}, \sigma, l, \rho, \mathbb{1}, \varrho}^{V, \mathbb{s}}\left(\widehat{\Sigma}, X, L, \mathbf{d}^{\prime}, d^{\prime \prime}, 0\right)}=\operatorname{det}\left(D^{\#}\right) \otimes \bigotimes_{a, i} e v b_{a i}^{*} \operatorname{det}(T L), \\
\mathcal{T}_{1}=\operatorname{det}\left(D^{\prime}\right) \otimes \bigotimes_{a_{1}, i_{1}}\left(e v b_{a_{1} i_{1}}^{1}\right)^{*} \operatorname{det}(T L), \\
\mathcal{T}_{2}=\operatorname{det}\left(D^{\prime \prime}\right) \otimes \bigotimes_{a_{2}, i_{2}}\left(e v b_{a_{2} i_{2}}^{2}\right)^{*} \operatorname{det}(T L)
\end{gathered}
$$

be the corresponding modified line bundles over moduli spaces $\widetilde{\mathcal{M}}_{\mathbf{k}, \sigma, l, \rho, \mathbb{1}, \varrho}^{V, \mathbb{s}}\left(\widehat{\Sigma}, X, L, \mathbf{d}^{\prime}, d^{\prime \prime}, 0\right)$. 
Lemma 5.3 For any $u=\left(u_{1}, u_{2}\right) \in \widetilde{\mathcal{M}}_{\mathbf{k}, \sigma, l, \rho, 1, \varrho}^{V, \mathbb{S}}\left(\widehat{\Sigma}, X, L, \mathbf{d}^{\prime}, d^{\prime \prime}, 0\right)$, there exists an isomorphism of modified determinant lines

$$
\left.\left.\left.\mathcal{T}\right|_{u} \cong(-1)^{\left(\left|\mathbf{k}_{1}\right|-1\right)\left(k_{2}-1\right)} \mathcal{T}_{1}\right|_{u} \otimes \mathcal{T}_{2}\right|_{u} \otimes e v b_{0}^{*} \operatorname{det}(T L)_{u}^{*} .
$$

which is orientation preserving.

Proof. Recall that we have two natural projections

$$
\begin{aligned}
& p_{1}: \widetilde{\mathcal{M}}_{\mathbf{k}, \sigma, l, \rho, \mathbb{1}, \varrho}^{V, \mathbb{s}}\left(\widehat{\Sigma}, X, L, \mathbf{d}^{\prime}, d^{\prime \prime}, 0\right) \rightarrow \widetilde{\mathcal{M}}_{\mathbf{k}_{1}, l_{1}, \mathbb{1}_{1}}^{V, \mathbb{1}^{1}}\left(\Sigma, X, L, \mathbf{d}^{\prime}, 0\right), \\
& p_{2}: \widetilde{\mathcal{M}}_{\mathbf{k}, \sigma, l, \rho, \mathbb{1}, \varrho}^{V, \mathbb{\Sigma}}\left(\widehat{\Sigma}, X, L, \mathbf{d}^{\prime}, d^{\prime \prime}, 0\right) \rightarrow \widetilde{\mathcal{M}}_{k_{2}, l_{2}, \mathbb{1}_{2}}^{V, \mathbb{s}^{2}}\left(D^{2}, X, L, d^{\prime \prime}, 0\right),
\end{aligned}
$$

and the bundle

$$
\mathcal{E}^{\#}:=p_{1}^{*} \mathcal{E}^{\prime} \oplus p_{2}^{*} \mathcal{E}^{\prime \prime}
$$

We define an operator

$$
\delta: p_{1}^{*} T \widetilde{\mathcal{M}}_{\mathbf{k}_{1}, l_{1}, \mathbb{1}_{1}}^{V,^{1}}\left(\Sigma, X, L, \mathbf{d}^{\prime}, 0\right) \oplus p_{2}^{*} T \widetilde{\mathcal{M}}_{k_{2}, l_{2}, \mathbb{1}_{2}}^{V, \mathbb{s}^{2}}\left(D^{2}, X, L, d^{\prime \prime}, 0\right) \rightarrow e v b_{0}^{*}(T L)
$$

by

$$
\xi^{\prime} \oplus \xi^{\prime \prime} \mapsto d\left(e v b_{0}^{\prime}\right)\left(\xi^{\prime}\right)-d\left(e v b_{0}^{\prime \prime}\right)\left(\xi^{\prime \prime}\right) .
$$

So it implies

$$
T \widetilde{\mathcal{M}}_{\mathbf{k}, \sigma, l, \rho, \mathbb{1}, \varrho}^{V, \mathbb{s}}\left(\widehat{\Sigma}, X, L, \mathbf{d}^{\prime}, d^{\prime \prime}, 0\right)=\operatorname{ker}(\delta) .
$$

We thus have a diagram of exact sequence

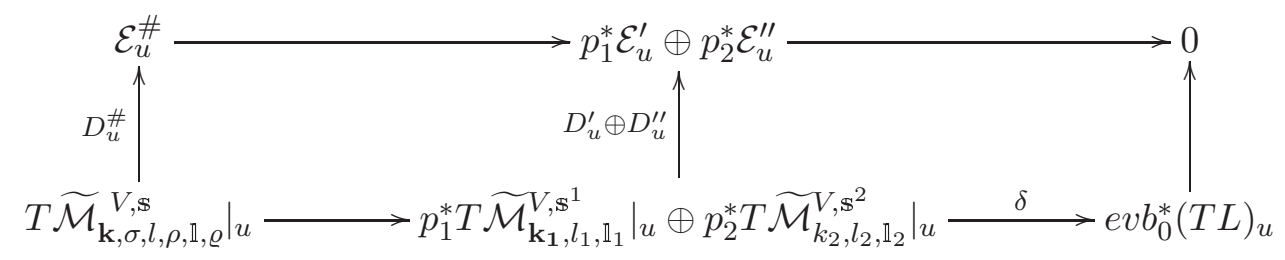

which implies an isomorphism

$$
\operatorname{det} D_{u}^{\#} \cong \operatorname{det} D_{u}^{\prime} \otimes \operatorname{det} D_{u}^{\prime \prime} \otimes e v b_{0}^{*} \operatorname{det}(T L)_{u}^{*} .
$$

Then (5.17) holds and the coefficient $(-1)^{\left(\left|\mathbf{k}_{1}\right|-1\right)\left(k_{2}-1\right)}$ records the change of cyclic order of boundary marked points.

Lemma 5.4 The gluing map

$$
\begin{gathered}
G l: \widetilde{\mathcal{M}}_{\mathbf{k}_{1}, l_{1}, \mathbb{1}_{1}}^{V, \mathbb{1}^{1}}\left(\Sigma, X, L, \mathbf{d}^{\prime}, 0\right)_{e v b_{z_{0}^{\prime}}} \times_{e v b_{z_{0}^{\prime \prime}}} \widetilde{\mathcal{M}}_{k_{2}, l_{2}, \mathbb{1}_{2}}^{V, \mathbb{s}^{2}}\left(D^{2}, X, L, d^{\prime \prime}, 0\right) \\
\longrightarrow \widetilde{\mathcal{M}}_{\mathbf{k}, l, \mathbb{1}}^{V, \mathbb{s}}\left(\Sigma, X, L, \mathbf{d}^{\prime}+d^{\prime \prime}, 0\right)
\end{gathered}
$$

is orientation preserving in the sense of the following isomorphism,

$$
\left.\left.\left.G l^{*} \mathcal{L}^{\prime}\right|_{u} \cong(-1)^{\left(\left|\mathbf{k}_{1}\right|-1\right)\left(k_{2}-1\right)} \mathcal{T}_{1}\right|_{u} \otimes \mathcal{T}_{2}\right|_{u},
$$

where $|\mathbf{k}|=\left|\mathbf{k}_{\mathbf{1}}\right|+k_{2}, l=l_{1}+l_{2}, \mathcal{L}^{\prime}$ is the bundle defined in (5.5). 
Proof. We claim that the argument is a combination of the conclusion of the lemma above and modification of ones in the lemma 8.3.5 and lemma 8.3.10 of FOOO which study the case of gluing of discs. The modification is direct, we will omit the detail. Note in our case we only assume relative $\operatorname{Pin}^{ \pm}$structure on $L$, one has to use the modified determinant lines to study the preserving of orientation.

Proposition 5.3 The gluing map (5.18) induces an isomorphism

$$
\begin{aligned}
& \partial \mathcal{M}_{\mathbf{k}, l, \mathbb{1}}^{V, \mathbb{s}}(\Sigma, X, L, \mathbf{d}, 0) \\
& \cong \bigcup(-1)^{*} \mathcal{M}_{\mathbf{k}_{1}+e_{b}, l_{1}, \mathbb{1}_{1}}^{V, \mathbb{1}^{1}}\left(\Sigma, X, L, \mathbf{d}^{\prime}, 0\right)_{e v b_{z_{0}^{\prime}}} \times_{e v b_{z_{0}^{\prime \prime}}} \mathcal{M}_{k_{2}+1, l_{2}, \mathbb{1}_{2}}^{V, \mathbb{s}^{2}}\left(D^{2}, X, L, d^{\prime \prime}, 0\right)
\end{aligned}
$$

compatible with orientations in the sense of the Lemma above, where the union is taken over all $b=1, \cdots, m, \mathbf{k}$ such that $|\mathbf{k}|=\left|\mathbf{k}_{1}\right|+k_{2}, l=l_{1}+l_{2}, \mathbb{1}=\mathbb{1}_{1}+\mathbb{1}_{2}$ and $\mathbf{d}=\mathbf{d}^{\prime}+d^{\prime \prime}, *=\left(\left|\mathbf{k}_{1}\right|-1\right)\left(k_{2}-1\right)+\left(n+\left|\mathbf{k}_{1}\right|\right)$.

Proof. The argument is parallel to the one in the Proposition 8.3.3 of [FOOO]. From definition we know that

$$
\begin{aligned}
& \mathcal{M}_{\mathbf{k}, l, \mathbb{1}}^{V, \mathbb{s}}(\Sigma, X, L, \mathbf{d}, 0) \cong \widetilde{\mathcal{M}}_{\mathbf{k}, l, \mathbb{1}}^{V, \mathbb{s}}(\Sigma, X, L, \mathbf{d}, 0) / \operatorname{Aut}\left(\Sigma_{\mathbf{d}}\right), \\
& \mathcal{M}_{\mathbf{k}_{1}+e_{b}, l_{1}, \mathbb{I}_{1}}^{V,,_{1}^{1}}\left(\Sigma, X, L, \mathbf{d}^{\prime}, 0\right) \cong \widetilde{\mathcal{M}}_{\mathbf{k}_{1}+e_{b}, l_{1}, \mathbb{1}_{1}}^{V, \mathbb{s}^{1}}\left(\Sigma, X, L, \mathbf{d}^{\prime}, 0\right) / \operatorname{Aut}\left(\Sigma_{\mathbf{d}^{\prime}}\right), \\
& \mathcal{M}_{k_{2}+1, l_{2}, \mathbb{1}_{2}}^{V, \mathbb{s}^{2}}\left(D^{2}, X, L, d^{\prime \prime}, 0\right) \cong \widetilde{\mathcal{M}}_{k_{2}+1, l_{2}, \mathbb{1}_{2}}^{V, \mathbf{s}^{2}}\left(D^{2}, X, L, d^{\prime \prime}, 0\right) / \operatorname{Aut}\left(D_{d^{\prime \prime}}^{2}\right) \text {. }
\end{aligned}
$$

We can take two boundary marked points $x_{0}$ and $x_{1}$ on the gluing component $(\partial \Sigma)_{b}$, and $y_{0}, y_{1}$ on $\partial D^{2}$. Denote by $\operatorname{Aut}\left(\Sigma ; x_{0}, x_{1}\right)\left(\operatorname{resp} . \operatorname{Aut}\left(D^{2} ; y_{0}, y_{1}\right)\right)$ the bi-holomorphic automorphisms group of $\Sigma$ fixing $x_{0}$ and $x_{1}$ (resp. $y_{0}$ and $y_{1}$ ). Then we have

$$
\mathbb{R}_{p} \times \operatorname{Aut}\left(\Sigma_{\mathbf{d}^{\prime}+d^{\prime \prime}} ; x_{0}, y_{1}\right) \cong \operatorname{Aut}\left(\Sigma_{\mathbf{d}^{\prime}} ; x_{0}, x_{1}\right) \times \operatorname{Aut}\left(D_{d^{\prime \prime}}^{2} ; y_{0}, y_{1}\right),
$$

where $\mathbb{R}_{p}$ is regarded as the space of gluing parameters. Note that

$$
\begin{aligned}
\widetilde{\mathcal{M}}_{\mathbf{k}-2 e_{b}, l, \mathbb{1}}^{V, \mathbb{1}}\left(\Sigma, X, L, \mathbf{d}^{\prime}+d^{\prime \prime}, 0\right) & \cong \mathcal{M}_{\mathbf{k}, l, \mathbb{1}}^{V, \mathbb{s}}\left(\Sigma, X, L, \mathbf{d}^{\prime}+d^{\prime \prime}, 0\right) \times \operatorname{Aut}\left(\Sigma_{\mathbf{d}^{\prime}+d^{\prime \prime}} ; x_{0}, y_{1}\right), \\
\widetilde{\mathcal{M}}_{\mathbf{k}_{1}-e_{b}, l_{1}, \mathbb{1}_{1}}^{V, \mathbb{s}^{1}}\left(\Sigma, X, L, \mathbf{d}^{\prime}, 0\right) & \cong \mathcal{M}_{\mathbf{k}_{1}+e_{b}, l_{1}, \mathbb{1}_{1}}^{V, \mathbb{s}^{1}}\left(\Sigma, X, L, \mathbf{d}^{\prime}, 0\right) \times \operatorname{Aut}\left(\Sigma_{\mathbf{d}^{\prime}} ; x_{0}, x_{1}\right), \\
\widetilde{\mathcal{M}}_{k_{2}-1, l_{2}, \mathbb{1}_{2}}^{V, \mathbb{s}^{2}}\left(D^{2}, X, L, d^{\prime \prime}, 0\right) & \cong \mathcal{M}_{k_{2}+1, l_{2}, \mathbb{1}_{2}}^{V, \mathbb{s}^{2}}\left(D^{2}, X, L, d^{\prime \prime}, 0\right) \times \operatorname{Aut}\left(D_{d^{\prime \prime}}^{2} ; y_{0}, y_{1}\right) .
\end{aligned}
$$

From (5.18) we have

$$
\begin{aligned}
& \widetilde{\mathcal{M}}_{\mathbf{k}-2 e_{b}, l, \mathbb{1}}^{V, \mathbb{s}}\left(\Sigma, X, L, \mathbf{d}^{\prime}+d^{\prime \prime}, 0\right) \\
& \cong \widetilde{\mathcal{M}}_{\mathbf{k}_{1}-e_{b}, l_{1}, \mathbb{1}_{1}}^{V, \mathbb{1}^{1}}\left(\Sigma, X, L, \mathbf{d}^{\prime}, 0\right)_{e v b_{z_{0}^{\prime}}} \times_{e v b_{z_{0}^{\prime \prime}}} \widetilde{\mathcal{M}}_{k_{2}-1, l_{2}, \mathbb{I}_{2}}^{V, \mathbb{s}^{2}}\left(D^{2}, X, L, d^{\prime \prime}, 0\right) .
\end{aligned}
$$


Then

$$
\begin{aligned}
& \mathcal{M}_{\mathbf{k}, l, \mathbb{1}}^{V, \mathbb{1}}\left(\Sigma, X, L, \mathbf{d}^{\prime}+d^{\prime \prime}, 0\right) \times \operatorname{Aut}\left(\Sigma_{\mathbf{d}^{\prime}+d^{\prime \prime}} ; x_{0}, y_{1}\right) \\
& \cong\left[\mathcal{M}_{\mathbf{k}_{1}+e_{b}, l_{1}, \mathbb{1}_{1}}^{V, \mathbb{s}^{1}}\left(\Sigma, X, L, \mathbf{d}^{\prime}, 0\right) \times \operatorname{Aut}\left(\Sigma_{\mathbf{d}^{\prime}} ; x_{0}, x_{1}\right)\right]_{e v b_{z_{0}^{\prime}}} \\
& \times_{e v b_{z_{0}^{\prime \prime}}}\left[\mathcal{M}_{k_{2}+1, l_{2}, \mathbb{1}_{2}}^{V, \mathbb{s}^{2}}\left(D^{2}, X, L, d^{\prime \prime}, 0\right) \times \operatorname{Aut}\left(D_{d^{\prime \prime}}^{2} ; y_{0}, y_{1}\right)\right] \\
& \cong(-1)^{n+\left|\mathbf{k}_{1}\right|} \operatorname{Aut}\left(\Sigma_{\mathbf{d}^{\prime}} ; x_{0}, x_{1}\right) \times\left[\mathcal{M}_{\mathbf{k}_{1}+e_{b}, l_{1}, \mathbb{I}_{1}}^{V, \mathbb{s}^{1}}\left(\Sigma, X, L, \mathbf{d}^{\prime}, 0\right)_{e v b_{z_{0}^{\prime}}}\right. \\
& \left.\times_{e v b_{z_{0}^{\prime \prime}}} \mathcal{M}_{k_{2}+1, l_{2}, \mathbb{1}_{2}}^{V, \mathbb{s}^{2}}\left(D^{2}, X, L, d^{\prime \prime}, 0\right)\right] \times \operatorname{Aut}\left(D_{d^{\prime \prime}}^{2} ; y_{0}, y_{1}\right)
\end{aligned}
$$

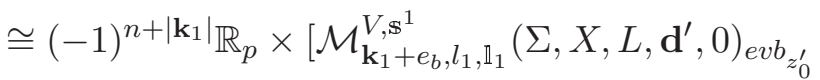

$$
\begin{aligned}
& \left.\times_{e v b_{z_{0}^{\prime \prime}}} \mathcal{M}_{k_{2}+1, l_{2}, \mathbb{1}_{2}}^{V, \mathbb{s}^{2}}\left(D^{2}, X, L, d^{\prime \prime}, 0\right)\right] \times \operatorname{Aut}\left(\Sigma_{\mathbf{d}^{\prime}+d^{\prime \prime}} ; x_{0}, y_{1}\right), \quad \text { by (5.21). }
\end{aligned}
$$

Therfore, the boundary components of $\mathcal{M}_{\mathbf{k}, l, \mathbb{1}}^{V, \mathbf{s}}(\Sigma, X, L, \mathbf{d}, 0)$ consist of

$$
\mathcal{M}_{\mathbf{k}_{1}+e_{b}, l_{1}, \mathbb{I}_{1}}^{V, \mathbb{s}^{1}}\left(\Sigma, X, L, \mathbf{d}^{\prime}, 0\right)_{e v b_{z_{0}^{\prime}}} \times_{e v b_{z_{0}^{\prime \prime}}} \mathcal{M}_{k_{2}+1, l_{2}, \mathbb{I}_{2}}^{V, \mathbf{s}^{2}}\left(D^{2}, X, L, d^{\prime \prime}, 0\right) .
$$

From the orientation defined above, the conclusion holds. $\square$

\section{Involution and sign}

Here the argument is different from the discussion by Ionel-Parker [IP1 for closed relative GW-invariant, we must deal with the codimension 1 frontier of the compactification of the moduli space of the open $V$-regular maps. We will modify the original method in [So]. Note in the sequel we still assume $L \cap V=\emptyset$.

Let us then suppose there exists an anti-symplectic involution $\phi$ such that $L=\operatorname{Fix}(\phi)$. And suppose $\Sigma$ is biholomorphic to its conjugation $\bar{\Sigma}$, i.e. there exists an anti-holomorphic involution $c: \Sigma \rightarrow \Sigma$. Fix $(J, \nu) \in \mathbb{J}_{\phi}^{V}$. Thus, from the $V$-regular $(J, \nu)$-holomorphic (resp. $\left.W^{1, p}\right)$ map $u:(\Sigma, \partial \Sigma) \mapsto(X, L)$ we can define its conjugate $(J, \nu)$-holomorphic (resp. $\left.W^{1, p}\right)$ map $\tilde{u}=\phi \circ u \circ c$ representing the homology class $\tilde{d}=[\tilde{u}]$. So we have an induced map

$$
\phi^{\prime}: B_{\mathbf{k}, l+\mathbb{1}}^{1, p, V}(X, L, \mathbf{d}) \rightarrow B_{\mathbf{k}, l+\mathbb{1}}^{1, p, V}(X, L, \tilde{\mathbf{d}})
$$

given by

$$
\mathbf{u}=(u, \vec{z}, \vec{w}, \vec{q}) \mapsto \tilde{\mathbf{u}}=\left(\tilde{u},\left(\left.c\right|_{\partial \Sigma}\right)^{|\mathbf{k}|}(\vec{z}), c^{l}(\vec{w}), c^{\mathbb{1}}(\vec{q})\right) .
$$

We denote the relevant Banach space bundle by $\widetilde{\mathcal{E}} \rightarrow B_{\mathbf{k}, l+\mathbb{1}}^{1, p, V}(X, L, \tilde{\mathbf{d}})$ with fiber

$$
\widetilde{\mathcal{E}}_{\tilde{\mathbf{u}}}:=L^{p}\left(\Sigma, \Omega^{0,1}\left(\tilde{u}^{*} T X\right)\right) .
$$

And we can similarly get a determinant line bundle of a family of Fredholm operators $\widetilde{D}: T B_{\mathbf{k}, l+\mathbb{1}}^{1, p, V}(X, L, \tilde{\mathbf{d}}) \rightarrow \widetilde{\mathcal{E}}$ as

$$
\widetilde{\mathcal{L}}:=\operatorname{det}(\widetilde{D}) \rightarrow B_{\mathbf{k}, l+\mathbb{1}}^{1, p, V}(X, L, \tilde{\mathbf{d}}) .
$$


The evaluation maps can also be similarly defined

$$
\begin{gathered}
\widetilde{e v} b_{a i}: B_{\mathbf{k}, l+\mathbb{1}}^{1, p, V}(X, L, \tilde{\mathbf{d}}) \rightarrow L, \quad i=1, \cdots, k_{a}, a=1, \cdots, m, \\
\widetilde{e v} i_{j}: B_{\mathbf{k}, l+\mathbb{1}}^{1, p, V}(X, L, \tilde{\mathbf{d}}) \rightarrow X, \quad j=1, \cdots, l, \\
\widetilde{e v} i_{\jmath}^{I}: B_{\mathbf{k}, l+\mathbb{1}}^{1, p, V}(X, L, \tilde{\mathbf{d}}) \rightarrow V, \quad \jmath=1, \cdots, \mathbb{1},
\end{gathered}
$$

by

$$
\widetilde{e v} b_{a i}(\tilde{\mathbf{u}})=\tilde{u}\left(c\left(z_{a i}\right)\right), \quad \widetilde{e v} i_{j}(\tilde{\mathbf{u}})=\tilde{u}\left(c\left(w_{j}\right)\right), \quad \widetilde{e v} i_{\jmath}^{I}(\tilde{\mathbf{u}})=\tilde{u}\left(c\left(q_{\jmath}\right)\right) .
$$

The total evaluation map is denoted by

$$
\begin{gathered}
\widetilde{\mathbf{e v}}:=\prod_{a, i} \widetilde{e v} b_{a i} \times \prod_{j} \widetilde{e v} i_{j} \times \prod_{\jmath} \widetilde{e v} i_{\jmath}^{I} \\
\widetilde{\mathbf{e v}}: B_{\mathbf{k}, l+l \mathbb{1}}^{1, p, V}(X, L, \tilde{\mathbf{d}}) \rightarrow L^{|\mathbf{k}|} \times X^{l} \times V^{\mathbb{1}} .
\end{gathered}
$$

Moreover, we can define a map

$$
\begin{gathered}
\Phi: \mathcal{E} \rightarrow \widetilde{\mathcal{E}} \\
\xi \mapsto d \phi \circ \xi \circ d c
\end{gathered}
$$

covering $\phi^{\prime}$. Also $\Phi$ induces a map $\Psi: \mathcal{L} \rightarrow \widetilde{\mathcal{L}}$ covering $\phi^{\prime}$. Denote by

$$
\widetilde{\mathcal{L}}^{\prime}:=\widetilde{\mathcal{L}} \otimes \bigotimes_{a, i} \widetilde{e v} b_{a i}^{*} \operatorname{det}(T L)^{*}
$$

Thus, $\Psi$ also induces a map

$$
\Psi^{\prime}: \mathcal{L}^{\prime} \rightarrow \widetilde{\mathcal{L}^{\prime}}
$$

covering $\phi^{\prime}$.

From the Proposition 5.1 and Definition 5.3 we know that both $\mathcal{L}^{\prime}$ and $\widetilde{\mathcal{L}^{\prime}}$ have canonical orientation. So the map $\Psi^{\prime}$ may either preserve the orientation component or reverse the orientation of some connected components. We say the sign of $\Psi^{\prime}$ is 0 if $\Psi^{\prime}$ preserves the orientation of $\mathcal{L}^{\prime}$ to that of $\widetilde{\mathcal{L}}^{\prime}$, otherwise, we say the sign of $\Psi^{\prime}$ is 1 . The following proposition shows an expression of the sign of $\Psi^{\prime}$. Recall $g_{0}$ denotes the genus of $\Sigma / \partial \Sigma$, and $\operatorname{dim} L=n$.

Proposition 6.1 If $L$ is relative Pin ${ }^{-}$, then the map $\Psi^{\prime}$ has sign

$$
\begin{aligned}
\mathfrak{s}^{-} \cong & \frac{\mu(d)(\mu(d)+1)}{2}+\left(1-g_{0}\right) n+n m+\operatorname{deg} \mathbb{\Phi}+|\mathbf{k}|+l+\mathbb{1} \\
& +w_{2}(\mathbb{V})(\psi(d))+w_{1}\left(u^{*} T L\right)(\partial d)+\sum_{a<b} w_{1}\left(u^{*} T L\right)\left(d_{a}\right) w_{1}\left(u^{*} T L\right)\left(d_{b}\right) \\
& +\sum_{a} w_{1}\left(u^{*} T L\right)\left(d_{a}\right)\left(k_{a}-1\right)+(n+1) \sum_{a} \frac{\left(k_{a}-1\right)\left(k_{a}-2\right)}{2} \quad \bmod 2 .
\end{aligned}
$$


If $L$ is relative Pin $^{+}$, then the map $\Psi^{\prime}$ has sign

$$
\begin{aligned}
\mathfrak{s}^{-} \cong & \frac{\mu(d)(\mu(d)+1)}{2}+\left(1-g_{0}\right) n+n m+\operatorname{deg} \mathbb{s}+|\mathbf{k}|+l+\mathbb{1} \\
& +w_{2}(\mathbb{V})(\psi(d))+\sum_{a<b} w_{1}\left(u^{*} T L\right)\left(d_{a}\right) w_{1}\left(u^{*} T L\right)\left(d_{b}\right) \\
& +\sum_{a} w_{1}\left(u^{*} T L\right)\left(d_{a}\right)\left(k_{a}-1\right)+(n+1) \sum_{a} \frac{\left(k_{a}-1\right)\left(k_{a}-2\right)}{2} \quad \bmod 2 .
\end{aligned}
$$

Where $\psi: H_{*}(X, L ; \mathbb{Z} / 2 \mathbb{Z}) \rightarrow H_{*}(X ; \mathbb{Z} / 2 \mathbb{Z})$ is a degree 0 homomorphism defined in [SO].

Since the proof of the preceding proposition is similar to the proof of the next one and we will not actually use it, we do not show the proof here.

Sometimes, for simplicity, we would denote $\mu=\mu(d), w_{2}=w_{2}(\mathbb{V})$ and $w_{1}=$ $w_{1}\left(u^{*} T L\right)$ if no danger of confusion.

Corollary 1 If $\operatorname{dim} L \leq 3$, then we always have

$$
\begin{aligned}
\mathfrak{s}^{-} \cong & \frac{\mu(\mu+1)}{2}+\left(1-g_{0}\right) n+n m+\operatorname{deg} \mathbb{s}+|\mathbf{k}|+l+\mathbb{1} \\
& +\sum_{a<b} w_{1}\left(d_{a}\right) w_{1}\left(d_{b}\right)+\sum_{a} w_{1}\left(d_{a}\right)\left(k_{a}-1\right) \\
& +(n+1) \sum_{a} \frac{\left(k_{a}-1\right)\left(k_{a}-2\right)}{2} \bmod 2 .
\end{aligned}
$$

In particular, if $\Sigma=D^{2}$, we have

$$
\begin{aligned}
\mathfrak{s}^{-} \cong & \frac{\mu(\mu-1)}{2}+\operatorname{deg} \mathbb{\Phi}+|\mathbf{k}|+l+\mathbb{1}+\mu(k-1) \\
& +(n+1) \frac{(k-1)(k-2)}{2} \bmod 2 .
\end{aligned}
$$

Proof. When $\operatorname{dim} L \leq 3$, the Wu relations imply that $L$ is $\operatorname{Pin}^{-}$, we can take the standard Pin $^{-}$structure. Note that $w_{2}(\mathbb{V})=0$ and $w_{1}(\partial d) \cong \mu(d) \bmod 2$, thus the formula is simplified.

Then we define and calculate the sign of a map related to the boundary of moduli space. Recall that

$$
\begin{aligned}
B^{V \#} & =B_{\mathbf{k}, \sigma, l, \rho, \mathbb{1}, \varrho}^{1, p, V}\left(\hat{\Sigma}, X, L, \mathbf{d}^{\prime}, d^{\prime \prime}, 0\right) \\
& :=B_{\mathbf{k}^{\prime}+e_{b}, l^{\prime}, \mathbb{1}^{\prime}}^{1, p}\left(\Sigma, X, L, \mathbf{d}^{\prime}, 0\right)_{e v b_{0}^{\prime}} \times_{e v b_{0}^{\prime \prime}} B_{k^{\prime \prime}+1, l^{\prime \prime}, \mathbb{1}^{\prime \prime}}^{1, p, V}\left(D^{2}, X, L, d^{\prime \prime}, 0\right) .
\end{aligned}
$$

Note that the standard conjugation $c: D^{2} \rightarrow D^{2}$ gives a biholomorphic isomorphism $D^{2} \simeq \bar{D}^{2}$. Then from the involution $\phi$, we just have an induced map

$$
\phi_{B^{\prime \prime}}: B_{k^{\prime \prime}+1, l^{\prime \prime}, 1^{\prime \prime}}^{1, p, V}\left(D^{2}, X, L, d^{\prime \prime}, 0\right) \rightarrow B_{k^{\prime \prime}+1, l^{\prime \prime}, 1^{\prime \prime}}^{1, p, V}\left(D^{2}, X, L, \widetilde{d^{\prime \prime}}, 0\right)
$$


of the second factor of the fiber product. Then since $L=\operatorname{Fix}(\phi), \phi_{B^{\prime \prime}}$ induces an map of the whole fiber product

$$
\begin{gathered}
\phi_{B^{\#}}: B^{V \#} \rightarrow \widetilde{B}^{V \#} \\
B_{\mathbf{k}, \sigma, l, \rho, \mathbb{1}, \varrho}^{1, p, V}\left(\hat{\Sigma}, X, L, \mathbf{d}^{\prime}, d^{\prime \prime}, 0\right) \rightarrow B_{\mathbf{k}, \sigma, l, \rho, \mathbb{1}, \varrho}^{1, p, V}\left(\hat{\Sigma}, X, L, \mathbf{d}^{\prime}, \widetilde{d^{\prime \prime}}, 0\right) .
\end{gathered}
$$

We denote the relevant Banach space bundle by

$$
\mathcal{E}^{\#} \rightarrow B^{V \#}, \quad \widetilde{\mathcal{E}}^{\#} \rightarrow \widetilde{B}^{V \#} .
$$

And we can similarly get a determinant line bundle of a family of Fredholm operators $\widetilde{D}: T B^{V \#} \rightarrow \widetilde{\mathcal{E}}^{\#}$ as

$$
\widetilde{\mathcal{L}}^{\#}:=\operatorname{det}(\widetilde{D}) \rightarrow B_{\mathbf{k}, \sigma, l, \rho, 1, \varrho}^{1, p, V}\left(\hat{\Sigma}, X, L, \mathbf{d}^{\prime}, \widetilde{d}^{\prime \prime}, 0\right) .
$$

The obvious evaluation maps are denoted by $\widetilde{e v} i_{j}, \widetilde{e v} b_{a i}, \widetilde{e v} i_{\jmath}^{I}$. Denote by

$$
\widetilde{\mathcal{L}}^{\# \prime}:=\widetilde{\mathcal{L}}^{\#} \otimes \bigotimes_{a, i} \widetilde{e v} b_{a i}^{*} \operatorname{det}(T L)^{*}
$$

Similarly, we have induced map $\Phi^{\#}: \mathcal{E}^{\#} \rightarrow \widetilde{\mathcal{E}}^{\#}$ covering $\phi_{B^{\#}}$. Recall that the inhomogeneous term $\nu$ vanishes on bubble components, so it is $\phi$-invariant. Therefore, $\left.\bar{\partial}_{(J, \nu)}^{\#}\right|_{\mathcal{E} \#}$ and $\left.\bar{\partial}_{(J, \nu)}^{\#}\right|_{\widetilde{\mathcal{E}} \#}$ are two $\phi_{B}^{\#}-\Phi^{\#}$ equivariant sections. Consequently, $\phi_{B}$ and $\Phi^{\#}$ induce a map of determinant line bundles $\Psi^{\#}: \mathcal{L}^{\#} \rightarrow \widetilde{\mathcal{L}}^{\#}$ covering $\phi_{B}$. Thus, $\Psi^{\#}$ also induces a map

$$
\Psi^{\#^{\prime}}: \mathcal{L}^{\#^{\prime}} \rightarrow \widetilde{\mathcal{L}}^{\#^{\prime}}
$$

covering $\phi_{B^{\#}}$. If this map preserves orientation the sign of it is 0 , otherwise the sign is 1 .

Remark. If the homology class $d$ represented by $u$ is $\phi$-anti-invariant, that is, the stable map $u$ is real, then all the maps above are involutions of their respective objects. In particular, we only define and calculate the sign of $\Psi^{\#^{\prime}}: \mathcal{L}^{\#} \rightarrow \mathcal{L}^{\#}$.

For stating the formulae for the sign of $\Psi^{\#^{\prime}}$, we introduce some new notation. Let

$$
\begin{gathered}
\Upsilon^{(1)}\left(d^{\prime \prime}, k^{\prime \prime}\right): \cong\left(d^{\prime \prime}\right) k^{\prime \prime} \cong w_{1}\left(\partial d^{\prime \prime}\right) k^{\prime \prime}, \\
\Upsilon^{(2)}\left(d_{b}^{\prime}, d^{\prime \prime}, k^{\prime}, k^{\prime \prime}\right): \begin{cases}0, & w_{1}\left(d_{b}^{\prime}\right)=w_{1}\left(\partial d^{\prime \prime}\right)=0, \\
k^{\prime}, & w_{1}\left(d_{b}^{\prime}\right)=w_{1}\left(\partial d^{\prime \prime}\right)=1, \\
k^{\prime \prime}-1, & w_{1}\left(d_{b}^{\prime}\right)=1, w_{1}\left(\partial d^{\prime \prime}\right)=0, \\
k^{\prime}+k^{\prime \prime}-1, & w_{1}\left(d_{b}^{\prime}\right)=0, w_{1}\left(\partial d^{\prime \prime}\right)=1 .\end{cases}
\end{gathered}
$$


Proposition 6.2 (1) Suppose the marked point $z_{b 1}$ does not bubble off, i.e. $1 \notin \sigma$. Then the map $\Psi^{\#^{\prime}}$ has sign

$$
\begin{aligned}
\mathfrak{s}_{ \pm}^{\#(1)} \cong & \frac{\mu\left(d^{\prime \prime}\right)\left(\mu\left(d^{\prime \prime}\right) \pm 1\right)}{2}+\operatorname{deg} \mathbb{s}^{\prime \prime}+k^{\prime \prime}+1+l^{\prime \prime}+\mathbb{1}^{\prime \prime} \\
& +w_{2}(\mathbb{V})\left(\psi\left(d^{\prime \prime}\right)\right)+\Upsilon^{(1)}\left(d^{\prime \prime}, k^{\prime \prime}\right) \\
& +(n+1) \frac{k^{\prime \prime}\left(k^{\prime \prime}-1\right)}{2} \bmod 2
\end{aligned}
$$

with + in the Pin $^{+}$and - in the Pin ${ }^{-}$case.

(2) Suppose the marked point $z_{b 1}$ bubbles off, i.e. $1 \in \sigma$. Then the map $\Psi^{\#^{\prime}}$ has sign

$$
\begin{aligned}
\mathfrak{s}_{ \pm}^{\#(2) \cong} & \frac{\mu\left(d^{\prime \prime}\right)\left(\mu\left(d^{\prime \prime}\right) \pm 1\right)}{2}+\operatorname{deg} \Phi^{\prime \prime}+k^{\prime \prime}+1+l^{\prime \prime}+\mathbb{1}^{\prime \prime} \\
& +w_{2}(\mathbb{V})\left(\psi\left(d^{\prime \prime}\right)\right)+\Upsilon^{(2)}\left(d_{b}^{\prime}, d^{\prime \prime}, k^{\prime}, k^{\prime \prime}\right)+w_{1}\left(d_{b}^{\prime}\right) w_{1}\left(\partial d^{\prime \prime}\right) \\
& +(n+1)\left[\frac{\left(k^{\prime \prime}-1\right)\left(k^{\prime \prime}-2\right)}{2}+k_{b}\left(k^{\prime \prime}+1\right)\right] \quad \bmod 2,
\end{aligned}
$$

with + in the Pin ${ }^{+}$and - in the Pin ${ }^{-}$case.

Remark. If $L$ is orientable and $\operatorname{dim} L=n$ is odd, note the fact that if $L$ is orientable then $\mu\left(d^{\prime \prime}\right)$ is even, so $w_{1}\left(d_{b}^{\prime}\right)=w_{1}\left(\partial d^{\prime \prime}\right)=0$, then we have

$$
\begin{aligned}
\mathfrak{s}^{\#(1)} & =\mathfrak{s}^{\#(2)} \\
& \cong \frac{\mu\left(d^{\prime \prime}\right)}{2}+\operatorname{deg} \mathbb{s}^{\prime \prime}+k^{\prime \prime}+1+l^{\prime \prime}+\mathbb{1}^{\prime \prime}+w_{2}(\mathbb{V})\left(\psi\left(d^{\prime \prime}\right)\right) \quad \bmod 2 .
\end{aligned}
$$

Proof of Proposition 6.2. The first two terms in $\mathfrak{s}_{ \pm}^{\#(i)}$ come from the formula (A.7). It is the sign of the conjugation of the determinant line associated with the restricted Pin boundary problem $D_{\mathbf{u}}^{\prime \prime} \oplus D_{0}^{\prime \prime}$ appearing in (5.15), induced from the conjugation on the moduli space of unmarked discs. The terms $k^{\prime \prime}+1+l^{\prime \prime}+\mathbb{1}^{\prime \prime}$ account for conjugation on the configuration space of marked points, satisfying an extra incidence condition. The term $w_{2}(V)\left(\psi\left(d^{\prime \prime}\right)\right)$ accounts for the change of orientation of the determinant line $\operatorname{det}\left(D_{\mathbf{u}}^{\prime \prime} \oplus D_{0}^{\prime \prime}\right)$ arising from the change of Pin structure induced by the involution $\phi$. Recall that the unique oriented path from $z \neq z_{0}$ to $z^{\prime}$ in the boundary of $\partial \widehat{\Sigma}$, induced by the complex structure of $\widehat{\Sigma}$, is very important for determining the canonical orientation of $\mathcal{L}^{\#^{\prime}}$. This path will reverse under conjugation. The terms $\Upsilon^{(1)}\left(\right.$ resp. $\left.\Upsilon^{(2)}+w_{1}\left(d_{b}^{\prime}\right) w_{1}\left(\partial d^{\prime \prime}\right)\right)$ in $\mathfrak{s}^{\#(1)}$ (resp. $\left.\mathfrak{s}^{\#(2)}\right)$ reflect this dependence. Note that reordering of the boundary marked points will affect the isomorphism in Definition 5.5 when the dimension of $L$ is even. The last terms account for this dependence.

\section{Equivalent definition of relatively open invariants}

From the discussion before, we see that we have two moduli spaces of $V$ regular maps $\mathcal{M}_{\mathbf{k}, l, \mathbb{1}}^{V, \mathbb{s}}(\Sigma, X, L, \mathbf{d})$ and, corresponding to the anti-symplectic involution $\phi, \mathcal{M}_{\mathbf{k}, l, \mathbb{1}}^{V, \mathbb{s}}(\Sigma, X, L, \tilde{\mathbf{d}})$. And we can restrict the two total evaluation maps 
(5.3) and (6.1) to have

$$
\begin{aligned}
& \text { ev }: \mathcal{M}_{\mathbf{k}, l, \mathbb{1}}^{V, \mathbb{s}}(\Sigma, X, L, \mathbf{d}) \rightarrow L^{|\mathbf{k}|} \times X^{l} \times V^{\mathbb{I}}, \\
& \widetilde{\mathbf{e v}}: \mathcal{M}_{\mathbf{k}, l, \mathbb{1}}^{V, \mathbb{\mathbb { N }}}(\Sigma, X, L, \tilde{\mathbf{d}}) \rightarrow L^{|\mathbf{k}|} \times X^{l} \times V^{\mathbb{1}} .
\end{aligned}
$$

For generic choice of points $\vec{x}=\left(x_{a i}\right), x_{a i} \in L$, and pairs of points $\vec{y}_{+}=\left(y_{j}^{+}\right)$, $\vec{y}_{-}=\left(y_{j}^{-}\right)$such that $y_{j}^{+}=\phi\left(y_{j}^{-}\right), j=1, \cdots, l$, and $\overrightarrow{\mathbb{q}}_{+}=\left(\mathbb{q}_{j}^{+}\right), \overrightarrow{\mathbb{q}}_{-}=\left(\mathbb{q}_{j}^{-}\right)$such that $\mathbb{q}_{j}^{+}=\phi\left(\mathbb{q}_{\jmath}^{-}\right), \mathbb{q}_{j}^{ \pm} \in V, \jmath=1, \cdots, \mathbb{l}$, the two total evaluation maps will be transverse to

$$
\prod_{a, i} x_{a i} \times \prod_{j} y_{j}^{+} \times \prod_{j} \mathbb{q}_{j}^{+} \text {and } \prod_{a, i} x_{a i} \times \prod_{j} y_{j}^{-} \times \prod_{j} \mathbb{q}_{\jmath}^{-}
$$

in $L^{|\mathbf{k}|} \times X^{l} \times V^{\mathbb{1}}$, respectively. For defining invariants, by index theorem, the following dimension condition would be satisfied

$$
(n-1)(|\mathbf{k}|+2 l)+(n-2) \cdot 2 \mathbb{l}=n(1-g)+\mu(d)-2 \operatorname{deg} \Phi-\operatorname{dim} \operatorname{Aut}(\Sigma),
$$

where $\mu: H_{2}(X, L) \rightarrow \mathbb{Z}$ denote the Maslov index, $g$ denote the genus of the closed Riemann surface $\Sigma \cup_{\partial \Sigma} \bar{\Sigma}$ obtained by doubling $\Sigma$. Note that $\mu(d)=\mu(\tilde{d})$, we can define a number as

$$
M(V, \mathbf{d}, \phi, \mathbf{k}, l, \mathbb{1})=\# \mathbf{e v}^{-1}\left(\vec{x}, \vec{y}_{+}, \overrightarrow{\mathbb{q}}_{+}\right)+\# \widetilde{\mathbf{e v}}^{-1}\left(\vec{x}, \vec{y}_{-}, \overrightarrow{\mathbb{q}}_{-}\right),
$$

where \# denotes the signed count with the sign of a given point, for example $\mathbf{u} \in \mathbf{e v}^{-1}\left(\vec{x}, \vec{y}_{+}, \overrightarrow{\mathbb{q}}_{+}\right)$, depending on whether or not the isomorphism

$$
\operatorname{dev}_{\mathbf{u}}: \operatorname{det}\left(T \mathcal{M}_{\mathbf{k}, l, \mathbb{1}}^{V, \mathbf{s}}(\Sigma, X, L, \mathbf{d})\right)_{\mathbf{u}} \simeq \mathbf{e v}^{*} \operatorname{det}\left(T\left(L^{|\mathbf{k}|} \times X^{l} \times V^{\mathbb{1}}\right)\right)_{\mathbf{u}}
$$

agrees with the underlying canonical isomorphism appearing in the Theorem 1.1

$$
\operatorname{det}\left(T \mathcal{M}_{\mathbf{k}, l, \mathbb{1}}^{V, \mathbf{s}}(\Sigma, X, L, \mathbf{d})\right) \underset{\longrightarrow}{\longrightarrow} \bigotimes_{a, i} e v b_{a i}^{*} \operatorname{det}(T L),
$$

up to the action of $\mathbb{R}^{+}$.

In particular, if $d=\tilde{d}$ we just simply define the number

$$
\mathscr{R} \mathscr{N}:=\mathscr{R} \mathscr{N}(V, \mathbf{d}, \mathbf{k}, l, \mathbb{I})=M(V, \mathbf{d}, \phi, \mathbf{k}, l, \mathbb{1})=\# \mathbf{e v}^{-1}(\vec{x}, \vec{y}, \overrightarrow{\mathbb{q}}),
$$

where $(\vec{x}, \vec{y}, \overrightarrow{\mathbb{q}})$ is a real configuration, i.e. $l=2 c, \vec{y}=\left\{y_{1}^{+}, \cdots, y_{c}^{+}, y_{1}^{-}, \cdots, y_{c}^{-}\right\}$ satisfying $y_{j^{\prime}}^{+}=\phi\left(y_{j^{\prime}}^{-}\right), j^{\prime}=1, \cdots, c ;$ and $\mathbb{1}=2 \mathbb{C}, \overrightarrow{\mathbb{q}}=\left\{\mathbb{q}_{1}^{+}, \cdots, \mathbb{q}_{\mathbb{C}}^{+}, \mathbb{q}_{1}^{-}, \cdots, \mathbb{q}_{\mathbb{C}}^{-}\right\}$ satisfying $\mathbb{q}_{\jmath^{\prime}}^{+}=\phi\left(\mathbb{q}_{\jmath^{\prime}}^{-}\right), \jmath^{\prime}=1, \cdots, \mathbb{C}$.

Remark. For such special $d \in H_{2}(X, L)$, we can prove that $\mathscr{R} \mathscr{N}$ is an invariant if $L$ is orientable and $\operatorname{dim} L \leq 3$ or if $L$ might not be orientable and $\operatorname{dim} L=2$. However, for general homology class $d$, we might not expect that $M(V, \mathbf{d}, \phi, \mathbf{k}, l, \mathbb{1})$ is invariant. In the sequel, we will construct invariants for general homology class $d$, the proof of invariance of $\mathscr{R} \mathscr{N}(V, \mathbf{d}, \mathbf{k}, l, \mathbb{1})$ can be considered as a special case 
of the proof below of the invariance of more general invariants.

In order to define relatively open invariants for general homology class $d \in$ $H_{2}(X, L)$, let us introduce more necessary notations. We denote by $\mathbb{d}=d_{\mathbb{C}}$ the doubling of $d$. For any homology class $\beta \in H_{2}(X, L)$, denote

$$
\bar{\beta}=\left(\beta, \beta_{1}, \cdots, \beta_{m}\right) \in H_{2}(X, L) \oplus H_{1}(L)^{\oplus m},
$$

and we denote the set of $(\mathbf{k}, l+\mathbb{1})$-real configurations by $\mathcal{R}:=\mathcal{R}(\vec{x}, \vec{y}, \overrightarrow{\mathbb{q}})$ which is

$$
\left\{\begin{array}{c}
\vec{r}=(\vec{x}, \vec{\xi}, \vec{\lambda})=\left(x_{11}, \cdots, x_{m k_{m}}, \xi_{1}, \cdots, \xi_{l}, \lambda_{1}, \cdots, \lambda_{\mathbb{1}}\right) \\
\mid \xi_{j}=y_{j}^{+} \text {or } \xi_{j}=y_{j}^{-}, j=1, \cdots, l ; \lambda_{\jmath}=\mathbb{q}_{\jmath}^{+} \text {or } \lambda_{\jmath}=\mathbb{q}_{\jmath}^{-}, \jmath=1, \cdots, \mathbb{1} .
\end{array}\right\} .
$$

Moreover, denote by $\mathbf{e v}_{(\beta, \vec{r})}$ the total evaluation map

$$
\begin{gathered}
\operatorname{ev}_{(\beta, \vec{r})}: \mathcal{M}_{\mathbf{k}, l, \mathbb{1}}^{V, \mathbb{s}}(X, L, \bar{\beta}) \rightarrow L^{|\mathbf{k}|} \times X^{l} \times V^{\mathbb{1}}, \\
(u, \vec{z}, \vec{w}, \vec{q}) \mapsto(\vec{x}=u(\vec{z}), \vec{\xi}=u(\vec{w}), \vec{\lambda}=u(\vec{q})) .
\end{gathered}
$$

Now, we can rewrite the number of (1.4) as

$$
\mathcal{I}:=\mathcal{I}_{X, \phi, g, \mathbb{d}, \mathbf{k}, l}^{V, \mathbb{x}}(\vec{x}, \vec{y}, \overrightarrow{\mathbb{q}})=\sum_{\forall \beta: \beta_{\mathbb{C}}=\mathbb{d} ; \forall \vec{r} \in \mathcal{R}} \# \mathbf{e v}_{(\beta, \vec{r})}^{-1}(\vec{x}, \vec{\xi}, \vec{\lambda})
$$

To show that the definition (7.5) is independent of the choices of $\vec{x}$ and pairs $\left(\vec{y}_{+}, \vec{y}_{-}\right),\left(\overrightarrow{\mathfrak{q}}_{+}, \overrightarrow{\mathbb{q}}_{-}\right)$is equivalent to proving the expression (1.4) is independent of the choices of $\operatorname{det}(T L)$-valued $n$-forms $\alpha_{a i}$, pairs of $2 n$-forms $\left(\gamma_{j}^{+}, \gamma_{j}^{-}\right)$and pairs of $(2 n-2)$-forms $\left(\eta_{j}^{+}, \eta_{j}^{-}\right)$, where $\gamma_{j}^{ \pm}$represent the Poincaré dual of $y_{j}^{ \pm}$for $j=1, \cdots, l$, and $\eta_{j}^{ \pm}$represent the Poincaré dual of $\mathbb{q}_{j}^{ \pm}$for $\jmath=1, \cdots, \mathbb{1}$.

\section{Proof of invariance}

In the following, we will show that the numbers $\mathcal{I}$ (in particular, $\mathscr{R} \mathscr{N}$ ) are invariants, provided $L$ is orientable and $\operatorname{dim} L \leq 3$, if $L$ is nonorientable, we suppose that $\operatorname{dim} L=2$ and the number of boundary marked points satisfy some additional conditions.

Suppose that we are given different points of real configuration $\left(\overrightarrow{x^{\prime}}, \overrightarrow{y^{\prime}} \pm, \overrightarrow{\mathbb{q}^{\prime}} \pm\right.$ satisfying the same generic conditions.

Let us denote

$$
\begin{gathered}
\mathbf{x}:[0,1] \rightarrow L^{|\mathbf{k}|}, \quad \mathbf{x}(0)=\vec{x}, \quad \mathbf{x}(1)=\overrightarrow{x^{\prime}} \\
\mathbf{y}^{ \pm}:[0,1] \rightarrow X^{l}, \quad \mathbf{y}^{+}(t)=\phi\left(\mathbf{y}^{-}(t)\right), \\
\mathbf{y}^{ \pm}(0)=\vec{y}_{ \pm}, \quad \mathbf{y}^{ \pm}(1)={\overrightarrow{y^{\prime}}}_{ \pm} \\
\mathbf{q}^{ \pm}:[0,1] \rightarrow V^{\mathbb{1}}, \quad \mathbf{q}^{+}(t)=\phi\left(\mathbf{q}^{-}(t)\right), \\
\mathbf{q}^{ \pm}(0)=\overrightarrow{\mathbb{q}}, \quad \mathbf{q}^{ \pm}(1)=\overrightarrow{\mathbb{q}^{\prime}}{ }_{ \pm}
\end{gathered}
$$




$$
\begin{array}{lll}
\Xi:[0,1] \rightarrow X^{l}, & \Xi(0)=\vec{\xi}, & \Xi(1)=\overrightarrow{\xi^{\prime}}, \\
\Lambda:[0,1] \rightarrow V^{\mathbb{1}}, & \Lambda(0)=\vec{\lambda}, & \Lambda(1)=\overrightarrow{\lambda^{\prime}},
\end{array}
$$

moreover, we require that $\xi_{j}=y_{j}^{+}$(resp. $y_{j}^{-}$) if and only if $\xi_{j}^{\prime}=y_{j}^{\prime+}$ (resp. $y_{j}^{\prime-}$ ), $\lambda_{\jmath}=\mathbb{q}_{\jmath}^{+}\left(\right.$resp. $\left.\mathbb{q}_{\jmath}^{-}\right)$if and only if $\lambda_{\jmath}^{\prime}=\mathbb{q}_{\jmath}^{\prime+}\left(\right.$ resp. $\left.\mathfrak{q}_{\jmath}^{\prime-}\right)$. Denote the set of all paths by

$$
\mathbf{R}:=\mathbf{R}\left(\mathbf{x}, \mathbf{y}^{ \pm}, \mathbf{q}^{ \pm}\right)=\{(\mathbf{x}, \Xi, \Lambda)\}
$$

And denote

$$
\begin{gathered}
\mathcal{W}(\mathbf{x}, \Xi, \Lambda, \bar{\beta}):=\mathcal{M}_{\mathbf{k}, l, \mathbb{1}}^{V, \mathbf{s}}(X, L, \bar{\beta})_{\mathbf{e v}_{(\beta, \bar{r})}} \times{ }_{(\mathbf{x} \times \Xi \times \Lambda) \circ \triangle}([0,1]), \\
\mathcal{W}=\mathcal{W}\left(\mathbf{x}, \mathbf{y}^{ \pm}, \mathbf{q}^{ \pm}, \mathbb{d}\right):=\underbrace{}_{\substack{\beta: \beta_{\mathbb{C}}=\mathbb{d},(\mathbf{x}, \Xi, \Lambda) \in \mathbf{R}}} \mathcal{W}(\mathbf{x}, \Xi, \Lambda, \bar{\beta}) .
\end{gathered}
$$

Note that $\mathcal{W}$ gives a smooth oriented cobordism between

$$
\bigcup_{\forall \beta: \beta_{\mathbb{C}}=\mathbb{d} ; \forall \vec{r} \in \mathcal{R}(\vec{x}, \vec{y}, \overrightarrow{\mathbb{q}})} \operatorname{ev}_{(\beta, \vec{r})}^{-1}(\vec{x}, \vec{\xi}, \vec{\lambda})
$$

and

$$
\bigcup_{\forall \beta: \beta_{\mathbb{C}}=\mathbb{d} ; \forall \overrightarrow{r^{\prime}} \in \mathcal{R}\left(\overrightarrow{x^{\prime}}, \overrightarrow{y^{\prime}}, \overrightarrow{\mathbb{q}^{\prime}}\right)} \mathbf{e v}_{\left(\beta, \overrightarrow{r^{\prime}}\right)}^{-1}\left(\overrightarrow{x^{\prime}}, \overrightarrow{\xi^{\prime}}, \overrightarrow{\lambda^{\prime}}\right) .
$$

Since in general $\mathcal{W}$ is noncompact, in order to prove the invariance of $\mathcal{I}:=$

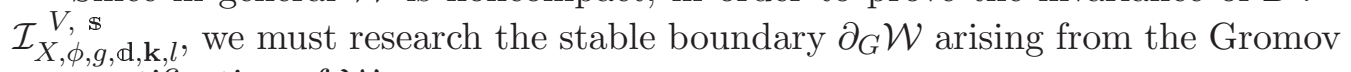
compactification of $\mathcal{W}$.

Note that for any tuple $\bar{\beta}=\left(\beta, \beta_{1}, \cdots, \beta_{m}\right) \in H_{2}(X, L) \oplus H_{1}(L)^{\oplus m}$ the boundary of $V$-stable compactification is

$$
\partial \overline{\mathcal{M}}_{\mathbf{k}, l, \mathbb{1}}^{V, \mathbb{s}}(\Sigma, X, L, \bar{\beta}):=\mathcal{M}_{\mathbf{k}, \sigma, l, \rho, \mathbb{1}, \varrho}^{V, \mathbb{s}}\left(\hat{\Sigma}, X, L, \bar{\beta}^{\prime}, \beta^{\prime \prime}, 0\right),
$$

where $\bar{\beta}^{\prime}=\left(\beta^{\prime}, \beta_{1}, \cdots, \beta_{b}^{\prime}, \cdots, \beta_{m}\right), \beta^{\prime}=\left[u_{1}(\Sigma)\right], \beta^{\prime \prime}=\left[u_{2}\left(D^{2}\right)\right] \in H_{2}(X, L)$, such that $\beta=\beta^{\prime}+\beta^{\prime \prime}, \partial \beta^{\prime \prime}+\beta_{b}^{\prime}=\beta_{b}$.

For simplicity, denote

$$
\begin{gathered}
\mathcal{M}(\Sigma)=\mathcal{M}_{\mathbf{k}^{\prime}+e_{b}, l^{\prime}, \mathbb{1}^{\prime}}^{V, \mathbb{s}}\left(\Sigma, X, L, \bar{\beta}^{\prime}, 0\right), \\
\mathcal{M}\left(D^{2}\right)=\mathcal{M}_{k^{\prime \prime}+1, l^{\prime \prime}, \mathbb{1}^{\prime \prime}}^{V, \mathbb{s}}\left(D^{2}, X, L, \beta^{\prime \prime}, 0\right) .
\end{gathered}
$$

Then

$$
\begin{aligned}
\left.\partial \overline{\mathcal{M}}_{\mathbf{k}, l, \mathbb{1}}^{V, \mathbb{s}}(\Sigma, X, L, \bar{\beta})\right) & =\mathcal{M}_{\mathbf{k}, \sigma, l, \rho, 1, \varrho}^{V, \mathbb{s}}\left(\hat{\Sigma}, X, L, \bar{\beta}^{\prime}, \beta^{\prime \prime}, 0\right) \\
& =\mathcal{M}(\Sigma)_{e v b_{z_{0}^{\prime}}} \times_{e v b_{z_{0}^{\prime \prime}}} \mathcal{M}\left(D^{2}\right) .
\end{aligned}
$$

We can generically choose $\left(\mathbf{x}, \mathbf{y}^{ \pm}, \mathbf{q}^{ \pm}\right)$such that $(\mathbf{x}, \Xi, \Lambda)=\left[\mathbf{x},\left(\Xi^{\prime}, \Xi^{\prime \prime}\right),\left(\Lambda^{\prime}, \Lambda^{\prime \prime}\right)\right] \in$ $\mathbf{R}$ is transverse to the total evaluation map

$$
\mathbf{e v}_{\left(\beta^{\prime}+\beta^{\prime \prime}, \vec{r}\right)}: \mathcal{M}_{\mathbf{k}, \sigma, l, \rho, \mathbb{1}, \varrho}^{V, \mathbb{s}}\left(\hat{\Sigma}, X, L, \bar{\beta}^{\prime}, \beta^{\prime \prime}, 0\right) \rightarrow L^{|\mathbf{k}|} \times X^{l} \times V^{\mathbb{1}} .
$$


We denote by

$$
\begin{aligned}
& \partial_{G} \mathcal{W}_{\sigma, \rho, \varrho, \beta=\beta^{\prime}+\beta^{\prime \prime}}^{b}\left(\Xi^{\prime \prime}, \Lambda^{\prime \prime}\right) \\
& \quad:=\mathcal{M}_{\mathbf{k}, \sigma, l, \rho, \mathbb{1}, \varrho}^{V, \mathbb{\Sigma}}\left(\hat{\Sigma}, X, L, \bar{\beta}^{\prime}, \beta^{\prime \prime}, 0\right)_{\mathbf{e v}_{\left(\beta^{\prime}+\beta^{\prime \prime}, \vec{r}\right)}} \times_{\left[\mathbf{x} \times\left(\Xi^{\prime}, \Xi^{\prime \prime}\right) \times\left(\Lambda^{\prime}, \Lambda^{\prime \prime}\right)\right] \circ \Delta}[0,1]
\end{aligned}
$$

the boundary stratum of the cobordism $\mathcal{W}$ arising from Gromov compactification. Denote

$$
\begin{aligned}
& \partial_{G} \mathcal{W}_{\sigma, \rho, \varrho, \beta^{\prime}, \beta^{\prime \prime}}^{b} \\
& \quad:=\partial_{G} \mathcal{W}_{\sigma, \rho, \varrho, \beta^{\prime}+\beta^{\prime \prime}}^{b}\left(\Xi^{\prime \prime}, \Lambda^{\prime \prime}\right) \bigcup \partial_{G} \mathcal{W}_{\sigma, \rho, \varrho, \beta^{\prime}+\widetilde{\beta^{\prime \prime}}}^{b}\left(\phi\left(\Xi^{\prime \prime}\right), \phi\left(\Lambda^{\prime \prime}\right)\right),
\end{aligned}
$$

and

$$
\partial_{G} \mathcal{W}_{\sigma, \rho, \varrho}^{b}=\bigcup_{\beta^{\prime}, \beta^{\prime \prime}:\left(\beta^{\prime}+\beta^{\prime \prime}\right)_{\mathbb{C}}=\mathbb{d}} \partial_{G} \mathcal{W}_{\sigma, \rho, \varrho, \beta^{\prime}, \beta^{\prime \prime}}^{b}
$$

Thus the total Gromov compactified boundary of $\mathcal{W}$ is

$$
\partial_{G} \mathcal{W}=\bigcup_{\substack{b \in[1, m], \sigma \subset\left[1, k_{b}\right], \rho \subset[1, l], \varrho \subset[1, \mathbb{1}]}}^{\bigcup} \partial_{G} \mathcal{W}_{\sigma, \rho, \varrho}^{b} .
$$

Then we have an induced involution map on the boundary of cobordism

$$
\begin{gathered}
\phi_{\partial}: \partial_{G} \mathcal{W} \rightarrow \partial_{G} \mathcal{W}, \\
\partial_{G} \mathcal{W}_{\sigma, \rho, \varrho, \beta^{\prime}+\beta^{\prime \prime}}^{b}\left(\Xi^{\prime \prime}, \Lambda^{\prime \prime}\right) \rightarrow \partial_{G} \mathcal{W}_{\sigma, \rho, \varrho, \beta^{\prime}+\widetilde{\beta^{\prime \prime}}}^{b}\left(\phi\left(\Xi^{\prime \prime}\right), \phi\left(\Lambda^{\prime \prime}\right)\right) .
\end{gathered}
$$

We claim that this map is fixed point free. Indeed, a fixed point of $\phi_{\partial}$ can only be in the strata satisfying $\beta^{\prime \prime}=\widetilde{\beta^{\prime \prime}}$. And we assume that there is no nonconstant $\phi$-multiply covered pseudoholomorphic disc. Since a $\phi$-somewhere injective disc can not be a fixed point of $\phi_{\partial}$, the fixed point of $\phi_{\partial}$ could only be the map that a zero energy disc bubbled off. The process of such bubbling-off corresponds to an interior marked point moving to the boundary. However, from the definition of moduli space (alternatively, we can also define the moduli space as a section of the reparameterization group action, see lemma 4.4 of $[$ So.) we know that the marked point constrained away from $L$ can not move to the boundary. So the contradiction implies that $\phi_{\partial}$ has no fixed point.

We below will show that $\phi_{\partial}$ is orientation reversing if

(i) $L$ is orientable, $\operatorname{dim} L=3$; or

(ii) $L$ might not be orientable, $\operatorname{dim} L=2$ and if $L$ is nonorientable we require the condition (5.6) is satisfied.

- Case (i). $L$ is orientable and $\operatorname{dim} L=3$ 
Since $\operatorname{dim} L=3$, by $\mathrm{Wu}$ relations, $L$ is $\operatorname{Pin}^{-}$, so $w_{2}(\mathbb{V})=0$. Since $L$ is orientable, $\mu\left(d^{\prime \prime}\right)$ is even. Note the assumption $L \cap V=\emptyset$. Therefore, by the formula (6.8), the sign of $\Psi^{\#^{\prime}}$ can be simplified as

$$
\mathfrak{s}^{\#}=\frac{\mu\left(\beta^{\prime \prime}\right)}{2}+\operatorname{deg} \mathbb{s}^{\prime \prime}+k^{\prime \prime}+1+l^{\prime \prime}+\mathbb{1}^{\prime \prime} \quad \bmod 2,
$$

Moreover, from the definition of the moduli space above we can see that the map

$$
\phi^{\#}: \mathcal{M}_{\mathbf{k}, \sigma, l, \rho, \mathbb{1}, \varrho}^{V, \mathbb{\Sigma}}\left(\hat{\Sigma}, X, L, \bar{\beta}^{\prime}, \beta^{\prime \prime}, 0\right) \rightarrow \mathcal{M}_{\mathbf{k}, \sigma, l, \rho, \mathbb{1}, \varrho}^{V, \mathbb{\Sigma}}\left(\hat{\Sigma}, X, L, \bar{\beta}^{\prime}, \widetilde{\beta^{\prime \prime}}, 0\right)
$$

has the same sign.

Note that the involution $\phi$ acts on $X$ reversing the orientation since $\phi^{*} \omega^{3}=$ $-\omega^{3}$. So $\phi_{\partial}$ acts non-trivially on $l^{\prime \prime}$ of the factors of $X^{l}$. Therefore, the sign of the map between the two fiber products

$$
\phi_{\partial}: \partial_{G} \mathcal{W}_{\sigma, \rho, \varrho, \beta^{\prime}+\beta^{\prime \prime}}^{b}\left(\Xi^{\prime \prime}, \Lambda^{\prime \prime}\right) \rightarrow \partial_{G} \mathcal{W}_{\sigma, \rho, \varrho, \beta^{\prime}+\widetilde{\beta^{\prime \prime}}}^{b}\left(\phi\left(\Xi^{\prime \prime}\right), \phi\left(\Lambda^{\prime \prime}\right)\right)
$$

should be independent of $l^{\prime \prime}$.

On the other hand, let us consider general dimension $n=\operatorname{dim} L$ for the moment. Recall (4.7) the virtual dimension of $V$-regular moduli space is

$$
\begin{aligned}
\operatorname{dim} \mathcal{M}_{\mathbf{k}, l, \mathbb{1}}^{V, \Phi}(\Sigma, X, L, \bar{\beta})= & \mu(\beta)+n(1-g)+|\mathbf{k}| \\
& +2(l+\mathbb{1}-\operatorname{deg} \Phi)-\operatorname{dim} A u t(\Sigma) .
\end{aligned}
$$

The definition of invariants requires the following dimension condition

$$
\mu(\beta)+n(1-g)+|\mathbf{k}|+2(l+\mathbb{1}-\operatorname{deg} \Phi)-\operatorname{dim} A u t(\Sigma)=(|\mathbf{k}|+2 l) n+2 \mathbb{1}(n-1) .
$$

So we have

$$
\mu(\beta)=(|\mathbf{k}|+2 l)(n-1)+2 \mathbb{I}(n-2)+2 \operatorname{deg} \Phi-n(1-g)+\operatorname{dim} A u t(\Sigma) .
$$

We observe that if we restrict the evaluation map to $L^{k^{\prime \prime}} \times X^{l^{\prime \prime}} \times V^{\mathbb{1}^{\prime \prime}}$, the image should be at least codimension one to have a nontrivial intersection. That is

$$
\begin{aligned}
\operatorname{dim} \mathcal{M}\left(D^{2}\right) & =n+\mu\left(\beta^{\prime \prime}\right)+\left(k^{\prime \prime}+2 l^{\prime \prime}\right)+2\left(\mathbb{1}^{\prime \prime}-\operatorname{deg} s^{\prime \prime}\right)-3 \\
& \geq\left(k^{\prime \prime}+2 l^{\prime \prime}\right) n+2 \mathbb{I}^{\prime \prime}(n-1)-1,
\end{aligned}
$$

therefore

$$
\mu\left(\beta^{\prime \prime}\right) \geq\left(k^{\prime \prime}+2 l^{\prime \prime}\right)(n-1)+2 \mathbb{I}^{\prime \prime}(n-2)+2 \operatorname{deg} \mathbb{s}^{\prime \prime}+2-n .
$$

Similarly, we have

$$
\begin{aligned}
\operatorname{dim} \mathcal{M}(\Sigma) & =n(1-g)+\mu\left(\beta^{\prime}\right)+\left(k^{\prime}+2 l^{\prime}\right)+2\left(\mathbb{1}^{\prime}-\operatorname{deg} \Phi^{\prime}\right)-\operatorname{dim} A u t(\Sigma) \\
& \geq\left(k^{\prime}+2 l^{\prime}\right) n+2 \mathbb{I}^{\prime}(n-1)-1,
\end{aligned}
$$


and so

$$
\begin{aligned}
\mu\left(\beta^{\prime}\right) \geq & \left(k^{\prime}+2 l^{\prime}\right)(n-1)+2 \mathbb{I}^{\prime}(n-2)+2 \operatorname{deg} s^{\prime} \\
& +\operatorname{dim} \operatorname{Aut}(\Sigma)-1-n(1-g) .
\end{aligned}
$$

Then from 8.7) and (8.11) we have

$$
\begin{aligned}
\mu\left(\beta^{\prime \prime}\right) & =\mu(\beta)-\mu\left(\beta^{\prime}\right) \\
& \leq\left(k^{\prime \prime}+2 l^{\prime \prime}\right)(n-1)+2 \mathbb{I}^{\prime \prime}(n-2)+2 \operatorname{deg} \Phi^{\prime \prime}+1 .
\end{aligned}
$$

In particular, when $n=3$, from (8.9) and (8.12), and noting that $\mu\left(\beta^{\prime \prime}\right)$ is even, we see that each set $\partial_{G} \mathcal{W}_{\sigma, \rho, \varrho, \beta^{\prime}+\beta^{\prime \prime}}^{b}\left(\Xi^{\prime \prime}, \Lambda^{\prime \prime}\right)$ is nonempty if and only if the following dimension condition is satisfied

$$
\mu\left(\beta^{\prime \prime}\right)=2\left(k^{\prime \prime}+2 l^{\prime \prime}\right)+2 \mathbb{1}^{\prime \prime}+2 \operatorname{deg} s^{\prime \prime} .
$$

Thus we have

$$
\operatorname{sign}\left(\phi_{\partial}\right)=\frac{\mu\left(\beta^{\prime \prime}\right)}{2}+\operatorname{deg} \Phi^{\prime \prime}+k^{\prime \prime}+1+\mathbb{1}^{\prime \prime} \cong 1 \quad(\bmod 2) .
$$

That is to say, the map $\phi_{\partial}$ reverses orientation. That means $\# \partial_{G} \mathcal{W}=0$. Therefore, we have

$$
\begin{aligned}
0=\# \partial \overline{\mathcal{W}} & =\sum_{\beta ; \overrightarrow{r^{\prime}}} \# \mathbf{e v}_{\left(\beta, \overrightarrow{r^{\prime}}\right)}^{-1}\left(\overrightarrow{x^{\prime}}, \overrightarrow{\xi^{\prime}}, \overrightarrow{\lambda^{\prime}}\right)-\sum_{\beta ; \vec{r}} \# \mathbf{e v}_{(\beta, \vec{r})}^{-1}(\vec{x}, \vec{\xi}, \vec{\lambda})+\# \partial_{G} \mathcal{W} \\
& =\mathcal{I}_{X, \phi, g, \mathbb{d}, \mathbf{k}, l}^{V, \mathbb{s}}\left(\overrightarrow{x^{\prime}}, \overrightarrow{y^{\prime}}, \overrightarrow{\mathbb{q}^{\prime}}\right)-\mathcal{I}_{X, \phi, g, \mathbb{d}, \mathbf{k}, l}^{V, \mathbf{s}}(\vec{x}, \vec{y}, \overrightarrow{\mathbb{q}}) .
\end{aligned}
$$

So integers $\mathcal{I}_{X, \phi, q, \mathbb{d}, \mathbf{k}, l}^{V, \mathbb{s}}$ are independent of the choice of $(\vec{x}, \vec{y}, \overrightarrow{\mathbb{q}})$. Equivalently, we can say that the integral in (1.4) is independent of the choices of $\alpha_{a i}, \gamma_{j}$ and $\eta_{j}$. Similarly, we can prove that $\mathcal{I}_{X, \phi, g, \mathbb{d}, \mathbf{k}, l}^{V, \mathbb{s}}$ are independent of the generic choice of $J \in \mathcal{J}_{\omega, \phi}$, and the choice of inhomogeneous perturbation $\nu \in \mathcal{P}_{\phi, c}$. That means $\mathcal{I}_{X, \phi, g, \mathbb{d}, \mathbf{k}, l}^{V, \Phi}$ are invariants of the tuple $(X, \omega, V, \phi)$. In particular, if $d=\tilde{d}$, the numbers $\mathscr{R} \mathscr{N}=M(V, \mathbf{d}, \phi, \mathbf{k}, l, \mathbb{1})$ in (7.3) are also invariants for this case.

- Case (ii). $\operatorname{dim} L=2$, and (5.6) is satisfied if $L$ is not orientable

Also by $\mathrm{Wu}$ relations, $L$ is $\mathrm{Pin}^{-}$, so $w_{2}(\mathbb{V})=0$. As the argument above, we conclude that the sign of the map $\phi^{\#}$ is given by formulas (6.6) and (6.7) depending on whether or not $1 \in \sigma$. Since $\phi^{*} \omega^{2}=\omega^{2}, \phi$ preserves the orientation of $X$. It means that (6.6) and (6.7) also coincide with the signs of the map $\phi_{\partial}$. Then by inequalities (8.9) and (8.12), note $n=2$, we see that the stratum $\partial_{G} \mathcal{W}_{\sigma, \rho, \varrho, \beta^{\prime}, \beta^{\prime \prime}}^{b}$ will be empty unless

$$
\mu\left(\beta^{\prime \prime}\right)+r=k^{\prime \prime}+2 l^{\prime \prime}+2 \operatorname{deg} \mathbb{s}^{\prime \prime}
$$


for $r=0$ or -1 . The following calculation shows that the signs (6.6) and (6.7) are exactly 1 .

First, assume $1 \notin \sigma$. Using the restriction (8.14), we have

$$
\begin{aligned}
\frac{k^{\prime \prime}\left(k^{\prime \prime}-1\right)}{2} & =\frac{\left(\mu\left(\beta^{\prime \prime}\right)+r-2 l^{\prime \prime}-2 \operatorname{deg} \mathbb{s}^{\prime \prime}\right)\left(\mu\left(\beta^{\prime \prime}\right)+r-2 l^{\prime \prime}-2 \operatorname{deg} \Phi^{\prime \prime}-1\right)}{2} \\
& \cong \frac{\mu\left(\beta^{\prime \prime}\right)\left(\mu\left(\beta^{\prime \prime}\right)-1\right)}{2}+\frac{r(r-1)}{2}+l^{\prime \prime}+\operatorname{deg} \Phi^{\prime \prime}+r \mu\left(\beta^{\prime \prime}\right) \quad(\bmod 2) .
\end{aligned}
$$

Also from (8.14), we have

$$
\begin{aligned}
\Upsilon^{(1)}\left(\beta^{\prime \prime}, k^{\prime \prime}\right) \cong \mu\left(\beta^{\prime \prime}\right) k^{\prime \prime} & \cong \mu\left(\beta^{\prime \prime}\right)^{2}+r \mu\left(\beta^{\prime \prime}\right)+2\left(l^{\prime \prime}+\operatorname{deg} \Phi^{\prime \prime}\right) \mu\left(\beta^{\prime \prime}\right) \\
& \cong \mu\left(\beta^{\prime \prime}\right)^{2}+r \mu\left(\beta^{\prime \prime}\right) \quad(\bmod 2) .
\end{aligned}
$$

Substituting (8.14), (8.15) and (8.16) into (6.6), we have

$$
\mathfrak{s}_{-}^{\#(1)} \cong \frac{r(r+1)}{2}+\mathbb{1}^{\prime \prime}+1 \quad(\bmod 2) .
$$

Also note that when $n=2$, the involution $\phi$ acts on 1 dimensional submanifold $V$ reversing the orientation since $\phi^{*} \omega=-\omega$. So $\phi_{\partial}$ acts non-trivially on $\mathbb{1}^{\prime \prime}$ of the factors of $V^{\mathbb{1}}$. Therefore, the sign of the map between the two fiber products

$$
\phi_{\partial}: \partial_{G} \mathcal{W}_{\sigma, \rho, \varrho, \beta^{\prime}+\beta^{\prime \prime}}^{b}\left(\Xi^{\prime \prime}, \Lambda^{\prime \prime}\right) \rightarrow \partial_{G} \mathcal{W}_{\sigma, \rho, \varrho, \beta^{\prime}+\widetilde{\beta^{\prime \prime}}}^{b}\left(\phi\left(\Xi^{\prime \prime}\right), \phi\left(\Lambda^{\prime \prime}\right)\right)
$$

should be independent of $\mathbb{1}^{\prime \prime}$. Thus we have

$$
\operatorname{sign}\left(\phi_{\partial}\right)=\frac{r(r+1)}{2}+1 \cong 1 \quad(\bmod 2)
$$

since $r=0$ or -1 .

Then we consider the case $1 \in \sigma$. Using the restriction (8.14) again, we have

$$
\begin{aligned}
\frac{\left(k^{\prime \prime}-1\right)\left(k^{\prime \prime}-2\right)}{2}= & \frac{\left(\mu\left(\beta^{\prime \prime}\right)+r-2 l^{\prime \prime}-2 \operatorname{deg} \Phi^{\prime \prime}-1\right)\left(\mu\left(\beta^{\prime \prime}\right)+r-2 l^{\prime \prime}-2 \operatorname{deg} \Phi^{\prime \prime}-2\right)}{2} \\
\cong & \frac{\mu\left(\beta^{\prime \prime}\right)\left(\mu\left(\beta^{\prime \prime}\right)+1\right)}{2}+\frac{r(r+1)}{2} \\
& +l^{\prime \prime}+\operatorname{deg} \Phi^{\prime \prime}+r \mu\left(\beta^{\prime \prime}\right)+1 \quad(\bmod 2) .
\end{aligned}
$$

Recall the condition (5.6): $w_{1}\left(\beta_{b}\right) \cong k_{b}+1$ and (8.14), we calculate

$$
k_{b}\left(k^{\prime \prime}+1\right) \cong\left(w_{1}\left(\beta_{b}\right)+1\right)\left(\mu\left(\beta^{\prime \prime}\right)+r+1\right) \quad(\bmod 2) .
$$

Substituting (8.14), (8.17) and (8.18) into (6.7), noting that $r=0$ or -1 , we have

$$
\begin{aligned}
\mathfrak{s}_{-}^{\#(2) \cong} & \frac{r(r+1)}{2}+r \mu\left(\beta^{\prime \prime}\right)+r+\mathbb{1}^{\prime \prime} \\
& +\left(w_{1}\left(\beta_{b}\right)+1\right)\left(\mu\left(\beta^{\prime \prime}\right)+r+1\right)+\Upsilon^{(2)}+w_{1}\left(\beta_{b}^{\prime}\right) w_{1}\left(\partial \beta^{\prime \prime}\right) \\
\cong & r \mu\left(\beta^{\prime \prime}\right)+r+\mathbb{1}^{\prime \prime}+\left(w_{1}\left(\beta_{b}\right)+1\right)\left(\mu\left(\beta^{\prime \prime}\right)+r+1\right) \\
& +\Upsilon^{(2)}+w_{1}\left(\beta_{b}^{\prime}\right) w_{1}\left(\partial \beta^{\prime \prime}\right)
\end{aligned}
$$


Recall the formula (6.5) and the fact

$$
w_{1}\left(\beta_{b}\right)=w_{1}\left(\beta_{b}^{\prime}\right)+w_{1}\left(\partial \beta^{\prime \prime}\right)
$$

we can express

$$
\Upsilon^{(2)}=w_{1}\left(\beta_{b}\right)\left(k^{\prime \prime}-1\right)+w_{1}\left(\partial \beta^{\prime \prime}\right) k^{\prime}
$$

Considering the fact that $\mu\left(\beta^{\prime \prime}\right) \cong w_{1}\left(\partial \beta^{\prime \prime}\right)(\bmod 2)$, and using (8.14) and (5.6) , we obtain

$$
\begin{aligned}
w_{1}\left(\partial \beta^{\prime \prime}\right) k^{\prime} & \cong w_{1}\left(\partial \beta^{\prime \prime}\right)\left(k_{b}-k^{\prime \prime}\right) \\
& \cong w_{1}\left(\partial \beta^{\prime \prime}\right)\left(w_{1}\left(\beta_{b}\right)+1+\mu\left(\beta^{\prime \prime}+r\right)\right) \\
& \cong w_{1}\left(\partial \beta^{\prime \prime}\right) w_{1}\left(\beta_{b}^{\prime}\right)+\mu\left(\beta^{\prime \prime}\right)(1+r) \quad(\bmod 2) .
\end{aligned}
$$

Substituting (8.21) and (8.14) in (8.20), we get

$$
\Upsilon^{(2)} \cong w_{1}\left(\beta_{b}\right)\left(\mu\left(\beta^{\prime \prime}\right)+r+1\right)+w_{1}\left(\partial \beta^{\prime \prime}\right) w_{1}\left(\beta_{b}^{\prime}\right)+\mu\left(\beta^{\prime \prime}\right)(1+r)(\bmod 2) .
$$

Substituting the last formula in (8.19), we calculate

$$
\begin{aligned}
& \mathfrak{s}_{-}^{\#(2)} \cong r \mu\left(\beta^{\prime \prime}\right)+r+\mathbb{1}^{\prime \prime}+\left(\mu\left(\beta^{\prime \prime}\right)+r+1\right)+\mu\left(\beta^{\prime \prime}\right)(1+r) \\
& \cong \mathbb{1}^{\prime \prime}+1 \quad(\bmod 2) .
\end{aligned}
$$

As the mentioned reason above, the sign of $\phi_{\partial}$ is independent of $\mathbb{1}^{\prime \prime}$, so $\operatorname{sign}\left(\phi_{\partial}\right) \cong$ $1(\bmod 2)$, which implies $\mathcal{I}_{X, \phi, g, \mathbb{d}, \mathbf{k}, l}^{V, \mathbb{s}}$ are invariants of the tuple $(X, \omega, V, \phi)$. In particular, the numbers $\mathscr{R} \mathscr{N} \stackrel{(\phi)}{=} M(V, \mathbf{d}, \phi, \mathbf{k}, l, \mathbb{1})$ in (7.3) are invariants of the tuple $(X, \omega, V, \phi)$.

\section{Appendix}

For the convenience of the reader, we review some definitions and important conclusions in $\mathrm{So}$ about the orientation of determinant of real linear CauchyRiemann operator. For our concrete problem of intersection of stable maps with a codimensional two symplectic submanifold, we state that parallel conclusions hold for some kind of restriction of Cauchy-Riemann operator.

We denote by $\Gamma$ an appropriate Banach space completion of the smooth section of a vector bundle. For a vector bundle $V \rightarrow B$, we denote by $\mathfrak{F}(V)$ the orthonormal frame bundle of $V$ which is a principal $O(n)$ bundle. Recall the fact that the Lie group $\operatorname{Spin}(n)$ is the central $\mathbb{Z} / 2 \mathbb{Z}$ extension of the special orthogonal group $S O(n)$, similarly, the two groups $\operatorname{Pin}^{+}(n)$ and $\operatorname{Pin}^{-}(n)$, although are topologically the same, are two different central extensions of $O(n)$.

Definition A.1 A $\operatorname{Pin}^{ \pm}(n)$ structure $\mathfrak{P}=(P, p)$ on a vector bundle $V \rightarrow B$ consists of principal $\operatorname{Pin}^{ \pm}(n)$ bundle $P \rightarrow B$ and a $\operatorname{Pin}^{ \pm}(n)-O(n)$ equivariant bundle map

$$
p: P \rightarrow \mathfrak{F}(V) .
$$

A map $\varphi: V \rightarrow V^{\prime}$ between vector bundles with Pin structure preserves Pin structure if there exists a lifting $\tilde{\varphi}$ such that the following diagram commutes 


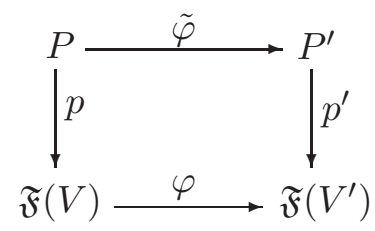

The obstruction to putting a Spin structure on a bundle $V \rightarrow B$ is $w_{2}(V) \in$ $H^{2}(B, \mathbb{Z} / 2 \mathbb{Z})$. For $\operatorname{Pin}^{+}$it is still $w_{2}(V)$, and for $\operatorname{Pin}^{-}$it is $w_{2}(V)+w_{1}^{2}(V)$.

Definition A.2 Let $(\Sigma, \partial \Sigma)$ be a Riemann surface with boundary $\partial \Sigma=\coprod_{a=1}^{m}(\partial \Sigma)_{a}$. A Cauchy-Riemann (or Riemann-Roch) Pin boundary value problem

$$
\underline{D}=(\Sigma, E, F, \mathfrak{P}, D)
$$

consists of

$1^{\circ}$ a complex vector bundle $E \rightarrow \Sigma$,

$2^{\circ}$ a totally real sub-bundle over the boundary $F \rightarrow \partial \Sigma$,

$3^{\circ}$ a Pin $^{+}$or Pin ${ }^{-}$structure $\mathfrak{P}$ on $F$,

$4^{\circ}$ an orientation of $\left.F\right|_{(\partial \Sigma)_{a}}$ for each a so that $\left.F\right|_{(\partial \Sigma)_{a}}$ is orientable,

$5^{\circ}$ a differential operator

$$
D: \Gamma((\Sigma, \partial \Sigma),(E, F)) \rightarrow \Gamma\left(\Sigma, \Omega^{0,1}(E)\right)
$$

such that for $f \in C^{\infty}(\Sigma, R), \xi \in \Gamma((\Sigma, \partial \Sigma),(E, F))$,

$$
D(f \xi)=f D \xi+(\bar{\partial} f) \xi .
$$

$D$ is called a real linear Cauchy-Riemann operator.

Remark. For the sake of studying the $(J, \nu)$-holomorphic maps relative to a codimensional 2 symplectic submanifold $V \subset X$, say $V$-regular maps, we may consider the restriction of a real linear Cauchy-Riemann operator $D$ to a subspace $\Gamma^{r e s t}:=\Gamma_{(\mathbb{s})}((\Sigma, \partial \Sigma),(E, F))$. A section $\xi \in \Gamma^{\text {rest }}$ (or $\left.\Gamma_{(\mathbb{s})}\right)$ if and only if it satidfies some vanishing conditions at each prescribed (say, intersection) marked point $p_{a \imath}$ (resp. $q_{\jmath}$ ). In the present paper, we only consider the orientability of moduli space under the condition $L \cap V=\emptyset$, thus we denote the subspace simply by $\Gamma^{r e s t}$ or $\Gamma_{\mathbb{S}}$ and the restriction of $D$ to this subspace by $D^{r e s t}$ or $D_{\mathbb{S}}$. In particular, for the concrete case of moduli space, $D_{\mathbb{s}}$ is the restriction by contact condition of $D_{u}=D_{u} \bar{\partial}_{(J, \nu)}$, where $u \in \mathcal{M}^{V}(X, L, d)$. When the pair $(J, \nu) \in \mathbb{J}^{V}$ (or $\mathbb{J}_{\phi}^{V}$ ) is $V$-compatible (see Definition 3.1), the arguments in Lemma 4.1 show that the restriction is transverse. Therefore, in such concrete situation $D_{\mathbb{s}}$ is $D_{u}$ for $u \in \mathcal{M}_{\mathrm{s}}^{V}(X, L, d)$.

It is not difficult to apply the arguments by Mcduff-Salamon (see [McS] Appendix C.2 ) to show that $D^{\text {rest }}$ is a Fredholm operator. We call $\underline{D^{\text {rest }}}=$ $\left(\Sigma, E, F, \mathfrak{P}, D^{\text {rest }}\right)$ the restricted Pin boundary value problem. 
For a Fredholm operator $D$, we define its determinant line by

$$
\operatorname{det}(D):=\Lambda^{\max }(\operatorname{ker} D) \otimes \Lambda^{\max }(\operatorname{coker} D)^{*} .
$$

As explained in [McS], for a family of Fredholm operators, $\operatorname{det}(D)$ denotes a line bundle with the natural topology.

Since we may trivialize $E$ over $\Sigma$ and each component of boundary $(\partial \Sigma)_{a} \simeq$ $S^{1}$, the restriction of $F$ to each $(\partial \Sigma)_{a}$ defines a loop of totally real subspaces of $\mathbb{C}^{n}$. it is well known such a loop associates a Maslov index $\mu_{a}$. we denote by

$$
\mu(E, F)=\sum_{a=1}^{m} \mu_{a}
$$

the total Maslov index of the vector bundle pair $(E, F)$. Moreover, $\mu(E, F)$ doesn't depend on the choice of trivialization of $E$.

We say $\underline{\varphi}: \underline{D} \rightarrow \underline{D}^{\prime}$ is an isomorphism of Cauchy-Riemann (or restricted) Pin boundary value problems if

i) there exists a biholomorphic map $f: \Sigma \rightarrow \Sigma^{\prime}$;

ii) there exists an isomorphism of bundles $\varphi: E \rightarrow E^{\prime}$ covering $f$ such that $\left.\varphi\right|_{\partial \Sigma}$ maps $F$ to $F^{\prime}$ and preserving $P$ in structure and preserving orientation if $F, F^{\prime}$ are orientable;

iii) $\varphi \circ D=D^{\prime} \circ \varphi$.

J. Solomon showed the following (Proposition 2.8 and Lemma 2.9 in [So]

Proposition A.1 The determinant line of a real-linear Cauchy-Riemann Pin boundary value problem $\underline{D}$ admits a canonical orientation. If $\underline{\varphi}: \underline{D} \rightarrow \underline{D^{\prime}}$ is an isomorphism, then the induced morphism

$$
\Psi: \operatorname{det}(D) \rightarrow \operatorname{det}\left(D^{\prime}\right)
$$

preserves the canonical orientation. Furthermore, the canonical orientation varies continuously in a family of Cauchy-Riemann operators. That is, it defines a single component of the determinant line bundle over that family. If the boundary condition $\left.F\right|_{(\partial \Sigma)_{a}}$ is orientable, then reversing the orientation on $\left.F\right|_{(\partial \Sigma)_{a}}$ will change the canonical orientation on $\operatorname{det}(D)$.

Remark. It is not difficult to apply the method of proof by Solomon to generalize the Proposition above to the case for the restricted Pin boundary value problem $\underline{D^{r e s t}}$. For instance, it is important in the proof of Proposition 2.8 in So that, for any chosen Cauchy-Riemann operator $\tilde{D}$ on the restriction $\left.\hat{E}\right|_{\tilde{\Sigma}}$, $\operatorname{det}(\tilde{D})$ has the canonical complex orientation, where $\hat{E} \rightarrow \hat{\Sigma}$ is the degenerated vector bundle of $E \rightarrow \Sigma, \hat{\Sigma}=\tilde{\Sigma} \cup_{a} \Delta_{a}\left(\tilde{\Sigma}\right.$ is the closed component, $\Delta_{a}$ is a disk corresponding to each $\left.(\partial \Sigma)_{a}\right)$. In our relative case, we consider the restriction $\tilde{D}^{\text {rest }}$ which is a complex Fredholm operator, and $\operatorname{det}\left(\tilde{D}^{\text {rest }}\right)$ also can be equipped with the canonical complex orientation. The remain arguments in the proof of Proposition 2.8 in $[\mathrm{So}$ can go through with minor modifications. 
We then come to study the sign of conjugation on the canonical orientation of the determinant line of a Cauchy-Riemann (or restricted) Pin boundary value problem. More precisely, given a Riemann surface $\Sigma$, let $\bar{\Sigma}$ denote the same topological surface with conjugate complex structure, and let

$$
c: \Sigma \rightarrow \bar{\Sigma}
$$

denote the tautological anti-holomorphic map. Similarly, let $(\bar{E}, \bar{F})$ denote the same real bundle pair with the opposite complex structure on $E$, and denote by

$$
C: E \rightarrow \bar{E}
$$

the tautological anti-complex-linear bundle map. Furthermore, a Cauchy-Riemann (resp. restricted) operator $D$ (resp. $D_{\mathbb{s}}$ ) on the bundle $E \rightarrow \Sigma$ is the same as a Cauchy-Riemann (resp. restricted) operator $\bar{D}$ (resp. $\bar{D}_{\mathbb{s}}$ ) on the bundle $\bar{E} \rightarrow \bar{\Sigma}$. So, given any Cauchy-Riemann (resp. restricted) Pin boundary problem $\underline{D}$ (resp. $\underline{D_{\mathbb{S}}}$ ), we may construct its conjugate $\underline{\bar{D}}$ (resp. $\bar{D}_{\mathbb{S}}$ ). Clearly, we have a tautological map of Cauchy-Riemann (resp. restricted) Pin boundary value problems

$$
\underline{C}: \underline{D} \rightarrow \underline{\bar{D}} \quad\left(\operatorname{resp} . \underline{D_{\mathbb{S}}} \rightarrow \underline{\bar{D}_{\mathbb{S}}}\right) .
$$

Suppose the genus of $\Sigma / \partial \Sigma$ is $g_{0}$, the number of boundary components of $\Sigma$ is $m$ and $\operatorname{rank}(F)=n$. Denote by $\mu=\mu(E, F)$ and by $w_{1}$ the first Stiefel-Whitney class. Solomon calculated the sign of the induced isomorphism $\Psi: \operatorname{det}(D) \rightarrow$ $\operatorname{det}(\bar{D})$ (Proposition 2.12 in $\underline{\underline{S o}}$ ).

Proposition A.2 The sign of the induced isomorphism $\Psi$ relative to the respective canonical orientation is given by

$$
\begin{aligned}
\operatorname{sign}^{+}(\underline{D})= & \frac{\mu(\mu+1)}{2}+\left(1-g_{0}\right) n+m n \\
& +\sum_{a<b} w_{1}(F)\left((\partial \Sigma)_{a}\right) w_{1}(F)\left((\partial \Sigma)_{b}\right) \quad \bmod 2,
\end{aligned}
$$

for a Pin $^{+}$structure and

$$
\begin{aligned}
& \operatorname{sign}^{-}(\underline{D})=\frac{\mu(\mu+1)}{2}+\left(1-g_{0}\right) n+m n \\
& +\sum_{a<b} w_{1}(F)\left((\partial \Sigma)_{a}\right) w_{1}(F)\left((\partial \Sigma)_{b}\right) \\
& +\sum_{a=1}^{m} w_{1}(F)\left((\partial \Sigma)_{a}\right) \quad \bmod 2,
\end{aligned}
$$

for a Pin ${ }^{-}$structure. In particular, when $\Sigma=D^{2}, g_{0}=0$ and $m=1$ we have

$$
\operatorname{sign}^{ \pm}(\underline{D})=\frac{\mu(\mu \pm 1)}{2} \quad \bmod 2 .
$$


As to our concrete case of regular maps, for the restricted Pin boundary value problem $D_{\mathbb{S}}$, we study the sign of the induced isomorphism $\Psi: \operatorname{det}\left(D_{\mathbb{S}}\right) \rightarrow$ $\operatorname{det}\left(\bar{D}_{\mathbb{s}}\right)$. Recall that when the pair $(J, \nu) \in \mathbb{J}^{V}$ (or $\mathbb{J}_{\phi}^{V}$ ), we can consider $D_{\mathbb{s}}=D_{u}$ for $u \in \mathcal{M}_{\mathbb{S}}^{V}(X, L, d)$, where $\mathbb{S}=\left(s_{1}, \cdots, s_{\mathbb{1}}\right)$ is the list of multiplicities of interior intersection points of a $V$-regular map, and $\operatorname{deg} \mathbb{S}=\sum_{\jmath=1}^{\mathbb{1}} s_{\jmath}$. The argument is a modification of the one of Proposition 2.12 in [So], involving the interior contact conditions. Basically, one need modify the formula (9) in So respecting to our concrete case of $V$-regular $(J, \nu)$-maps for generic $(J, \nu) \in \mathbb{J}^{V}$, the transversality of contact conditions (see Lemma 4.1) implies the relation

$$
\operatorname{index}_{\mathbb{C}}\left(\tilde{D}_{\mathbb{S}}\right)=\operatorname{index}_{\mathbb{C}}(\tilde{D})-\operatorname{deg}_{\mathbb{S}},
$$

where $\tilde{D}_{\mathbb{s}}$ is the restriction of the chosen complex Cauchy-Riemann operator $\tilde{D}$ on the restriction $\left.\hat{E}\right|_{\tilde{\Sigma}}$. Thus, we have

Proposition A.3 The sign of the induced isomorphism $\Psi: \operatorname{det}\left(D_{\mathbb{S}}\right) \rightarrow \operatorname{det}\left(\bar{D}_{\mathbb{S}}\right)$ relative to the respective canonical orientation is given by

$$
\begin{aligned}
\operatorname{sign}^{+}\left(\underline{D_{\Phi}}\right)= & \frac{\mu(\mu+1)}{2}+\operatorname{deg} \Phi+\left(1-g_{0}\right) n+m n \\
& +\sum_{a<b} w_{1}(F)\left((\partial \Sigma)_{a}\right) w_{1}(F)\left((\partial \Sigma)_{b}\right) \quad \bmod 2,
\end{aligned}
$$

for a Pin ${ }^{+}$structure and

$$
\begin{array}{rlr}
\left.\operatorname{sign}^{-} \underline{\underline{D_{\mathbf{S}}}}\right)= & \frac{\mu(\mu+1)}{2}+\operatorname{deg} \Phi+\left(1-g_{0}\right) n+m n \\
& +\sum_{a<b} w_{1}(F)\left((\partial \Sigma)_{a}\right) w_{1}(F)\left((\partial \Sigma)_{b}\right) & \\
& +\sum_{a=1}^{m} w_{1}(F)\left((\partial \Sigma)_{a}\right) & \bmod 2,
\end{array}
$$

for a Pin ${ }^{-}$structure. In particular, when $\Sigma=D^{2}, g_{0}=0$ and $m=1$ we have

$$
\operatorname{sign}^{ \pm}\left(\underline{D_{\Phi}}\right)=\frac{\mu(\mu \pm 1)}{2}+\operatorname{deg} \Phi \quad \bmod 2 .
$$

Sketch of proof. From Proposition A.1 and the remark after it, we see that the determinant line of the restricted real-linear Cauchy-Riemann Pin boundary value problem $\underline{D_{\Phi}}\left(\right.$ i.e. $\left.\underline{D^{\text {rest }}}\right)$ admits a canonical orientation. Note that the formula (7) in [So] can be modified as

$$
\operatorname{det}\left(\#_{a} D_{a} \# \tilde{D}_{\mathbb{S}}\right) \simeq \bigotimes_{a} \operatorname{det}\left(D_{a}\right) \otimes \operatorname{det}\left(\tilde{D}_{\mathbb{S}}\right) \otimes \bigotimes_{a} \operatorname{det}\left(E_{\hat{\gamma}_{a}}\right)^{*}
$$

Since conjugation on a complex vector space will make a sign change equal to its complex dimension $(\bmod 2)$, that changes the orientation of $\operatorname{det}\left(\tilde{D}_{\mathbb{s}}\right)$ according to the index $\operatorname{index}_{\mathbb{C}}\left(\tilde{D}_{\mathbb{S}}\right)=\operatorname{index}_{\mathbb{C}}(\tilde{D})-\operatorname{deg} \$$. Combining the formula (A.8) and the formula (9) and arguments in Proposition 2.12 of [So], one can verify the equalities (A.5), (A.6) and (A.7). 
Definition A.3 A short exact sequence of families of Fredholm operators

$$
0 \rightarrow D^{\prime} \rightarrow D \rightarrow D^{\prime \prime} \rightarrow 0
$$

consists of a base parameter space $B$, two short exact sequences of Banach space bundles over $B$

$$
0 \rightarrow X^{\prime} \rightarrow X \rightarrow X^{\prime \prime} \rightarrow 0, \quad 0 \rightarrow Y^{\prime} \rightarrow Y \rightarrow Y^{\prime \prime} \rightarrow 0 .
$$

and three Fredholm morphisms of Banach bundles

$$
D: X \rightarrow Y, \quad D^{\prime}: X^{\prime} \rightarrow Y^{\prime} \quad D^{\prime \prime}: X^{\prime \prime} \rightarrow Y^{\prime \prime},
$$

such that the following diagram commutes

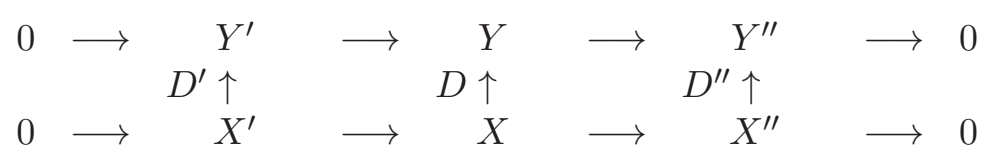

The following lemma will be used in section 8 .

Lemma A.1 A short exact sequence of families of Fredholm operators

$$
0 \rightarrow D^{\prime} \rightarrow D \rightarrow D^{\prime \prime} \rightarrow 0
$$

induces an isomorphism

$$
\operatorname{det}\left(D^{\prime}\right) \otimes \operatorname{det}\left(D^{\prime \prime}\right) \simeq \operatorname{det}(D) .
$$

\section{References}

[AG] N. Alling, N. Greenleaf, Foundations of the theory of Klein surfaces, Lecture Notes in Mathematics, Vol. 219, Springer-Verlag, Berlin-New York, 1971.

[AKV] M. Aganagic, A. Klemm, C. Vafa, Disk instantons, mirror symmetry and the duality web, Z.Naturforsch. A 57 (2002), No. 1-2, 1-28.

[C] C.-H. Cho, Counting real J-holomorphic discs and spheres in dimension four and six, ArXiv: math.SG/0604501.

[FOOO] K. Fukaya, Y.-G. Oh, H. Ohta and K. Ono, Lagrangian intersection Floer theory: anomaly and obstruction. Part I and II. AMS/IP Studies in Advanced Mathematics, 46. American Mathematical Society, Providence, RI; International Press, Somerville, MA, 2009.

[FO] K. Fukaya, K. Ono, Arnold conjecture and Gromov-Witten invariants for general symplectic manifolds, Topology 38 (1999), 933-1048.

[G] M. Gromov, Pseudoholomorphic curves in symplectic manifolds, Invent. Math. 82, (1985), 307-347. 
[H] H.-L. Her, Invariants for 6 Dimensional Symplectic Manifolds with Involution, Preprint.

[IP1] E. Ionel, T. Parker, Relative Gromov-witten invariants, Ann. of Math. 157 (2003), 45-96.

[IP2] E. Ionel, T. Parker, The symplectic sum formula for Gromov-Witten invariants, Ann. of Math. 159 (2004), 935-1025.

[KL] S. Katz, C.-C Liu, Enumerative geometry of stable maps with Lagrangian boundary conditions and multiple covers of the disc, Adv. Theor. Math. Phys. $\mathbf{5}$ (2001), No. 1, 1-49.

[KT] R. Kirby, L. Taylor, Pin structure on low-dimensional manifolds, Geometry of low-dimensional manifolds 2 (Durham, 1989), 177-242, London Math. Soc. Lecture Note ser., 151, Cambridge Univ. Press, Cambridge, 1990.

[KM] M. Kontsevich, Yu. Manin, Gromov-Witten classes, quantum cohomology, and enumerative geometry, Comm. Math. Phys. 164 (1994), no. 3, 525-562.

$[\mathrm{K}] \quad$ U. Koschorke, Infinite dimension K-theory and characteristic classes of Fredholm bundle maps, in Global Analysis, Proc. Symposia Pure Math. XV , A.M.S., Providence, R.I., 1970, 95-133.

[LR] A.-M. Li, Y. Ruan, Symplectic surgery and Gromov-Witten invariants of CalabiYau 3-folds, Invent. Math. 145 (2001), 151-218.

[LiT] J. Li, G. Tian, Virtual moduli cycles and Gromov-Witten invariants of general symplectic manifolds, in Topics in Symplectic 4-manifolds (Irvine, CA, 1996), 47-83, First Internat. Press Lect. Ser. I, International Press, Cambridge, MA, 1998.

[L] C.-C. Liu, Moduli of J-holomorphic curves with Lagrangian boundary conditions and open Gromov-Witten invariants for an $S^{1}$-equivariant pair, Preprint. arXiv:math.SG/0210257.

[LY] C.-H. Liu, S.-T. Yau, Degeneration and gluing of Kuranishi structures in Gromov-Witten theory and the degeneration/gluing axioms for open GromovWitten invariants under a symplectic cut, Preprint. ArXiv: math.SG/0609483.

[LiuT] G. Liu, G. Tian, Floer homology and Arnold conjecture, J. Diff. Geom. 49 (1998), 1-74.

[McS] D. McDuff, D. Salamon, J-holomorphic curves and symplectic topology, American Mathematical Society Colloquium Publications, 52, American Mathematical Society, Providence, RI, 2004.

[MS] J. Milnor, J, Stasheff, Characteristic classes, Annals of Mathematics Studies, No. 76, Princeton University Press, Princeton, N.J., 1974.

[OV] H. Ooguri, C. Vafa, Knot invariants and topological strings, Nuclear phys. B 577 (2000), No. 3, 419-438.

[PW] T. Parker, J. Wolfson, Pseudo-holomorphic maps and bubble trees, J. Geom. Anal. 3 (1993) 63-98. 
[R] Y. Ruan, Topological sigma model and Donaldson type invariants in Gromov theory, Duke Math J. 83 (1996), 461-500.

[RT1] Y. Ruan, G. Tian, A mathematical theory of quantum cohomology, J. Differential Geom. 42 , no. 2, (1995), 259-367.

[RT2] Y. Ruan, G. Tian, Higher genus symplectic invariants and sigma model coupled with gravity, Invent. Math. 130 (1997), 455-516.

[Si] B. Siebert, Gromov-Witten invariants for general symplectic manifolds, preprint, 1996. arXiv:dg-ga/9608005.

[Sil] V. de Silva, Products on symplectic Floer homology, Thesis, Oxford Uinv. 1997.

[So] J. Solomon, Intersection theory on the moduli space of holomorphic curves with Lagrangian boundary conditions, Preprint, arXiv:math/0610901.

[T] C. Taubes, Counting pseudo-holomorphic submanifolds in dimension 4, J. Diff. Grom. 44 (1996), 818-893.

[Ti] G. Tian, Quantum cohomology and its associativity, Current Developments in Mathematics 1995 (Cambridge, MX) 361-401, Inter. Press.

[W1] J.-Y. Welschinger, Invariants of real symplectic 4-manifolds and lower bounds in real enumerative geometry, Invent. Math., 162 (2005), 195-234.

[W2] J.-Y. Welschinger, Spinor states of real rationa; curves in real algebraic convex 3-manifolds and enumerative invariants, Duke Math. J. 127 (2005), No. 1, 89-121.

[W3] J.-Y. Welschinger, Enumerative invariants of stongly semipositive real symplectic six-manifolds, Preprint. 2007. arXiv:math/0509121v2.

[W4] J.-Y. Welschinger, Optimalité, congruences et calculs d'invariants des variétés symplectiques réelles de dimension quatre, Preprint, arXiv:0707.4317v1.

[W] E. Witten, Two dimensional gravity and intersection theory on moduli space, Surveys in Diff. Geom., 1 (1991), 243-310.

[Y] R. Ye, Gromov's compactness theorem for pseudoholomorphic curves, Trans. Amer. Math. Soc. 342 (1994), 671-694.

School of Mathematical Sciences

Nanjing Normal University

Nanjing 210023, P. R. China

Email: hailongher@126.com 